\title{
Removable sets for Lipschitz harmonic functions in the plane
}

\author{
Guy David and Pertti Mattila
}

\section{Introduction.}

The main motivation for this work comes from the century-old Painlevé problem: try to characterize geometrically removable sets for bounded analytic functions in $\mathbb{C}$. A compact set $E \subset \mathbb{C}$ is removable for bounded analytic functions if whenever $U$ is an open set containing $E$ and $f: U \backslash E \longrightarrow \mathbb{C}$ is bounded and analytic, then $f$ has an analytic extension to $U$. For other formulations and relations to analytic capacity, see, e.g., [G], [C1] or [M]. Painlevé proved that if $H^{1}(E)=0$ then $E$ is removable. Here $H^{1}$ is the one-dimensional Hausdorff (length) measure.

The present paper is the first of a series of two, but the second paper [D3] already appeared, thanks to a very fast publication process by the Revista, while the present paper was delayed by an attempt with some other journal. The authors are very grateful to the Revista for its very efficient handling of both papers. The main result in [D3] is the following.

Theorem 1.1. If $E \subset \mathbb{C}$ is compact and $H^{1}(E)<\infty$, then $E$ is removable for bounded analytic functions if and only if $E$ is purely unrectifiable, that is, $H^{1}(E \cap \Gamma)=0$ for every rectifiable curve $\Gamma$.

Although this result does not give a complete characterization, it only leaves out sets of infinite length and Hausdorff dimension 1, 
because sets of Hausdorff dimension bigger than 1 are not removable, see, e.g., $[\mathrm{G}]$.

The proof of Theorem 1.1 relies on the results and techniques of the present paper, plus a suitable generalization of the $T(b)$-Theorem to spaces with a measure that is not necessarily doubling. At the time this paper was written, we did not know how to prove such a theorem, and so we were only able to establish the analogue of Theorem 1.1 for Lipschitz harmonic functons.

A compact set $E \subset \mathbb{C}$ is removable for Lipschitz harmonic functions if whenever $U$ is an open set containing $E$ and $u: U \longrightarrow \mathbb{R}$ is a Lipschitz function which is harmonic in $U \backslash E$, then $u$ is harmonic in $U$. Every $E$ which is removable for bounded analytic functions is also removable for Lipschitz harmonic functions (use the fact that if $u$ is Lipschitz harmonic, then $\partial_{z} u$ is bounded analytic). But it is not known if these two classes are the same. Again sets of Hausdorff dimension bigger than 1 are not removable.

In this paper we shall prove:

Theorem 1.2. Let $E \subset \mathbb{C}$ be compact with $H^{1}(E)<\infty$. Then $E$ is removable for Lipschitz harmonic functions if and only if $E$ is purely unrectifiable.

For both theorems, only one direction needs to be proven because of the result due to Calderón and others, see, e.g., [C1]: if $E$ is a compact subset of a rectifiable curve with $H^{1}(E)>0$, then $E$ is not removable (for either class). Hence the removable sets are purely unrectifiable.

Theorem 1.1, and hence also Theorem 1.2, were proven in [MMV] for compact sets $E$ which are regular in the sense that there is a constant $C$ such that

$$
\frac{r}{C} \leq H^{1}(E \cap B(x, r)) \leq C r, \quad \text { for } x \in E, \quad 0<r \leq 1 .
$$

Here $B(x, r)$ is the closed disc with centre $x$ and radius $r$. Our proof of Theorem 1.2 will rely on some of the main ingredients of the method of [MMV], so we discuss it briefly here.

The proof in [MMV] was based on a relation between the Cauchy kernel $1 / z$ and the Menger curvature $c\left(z_{1}, z_{2}, z_{3}\right)$ of a triple $z_{1}, z_{2}$, $z_{3} \in \mathbb{C}$. By definition, $c\left(z_{1}, z_{2}, z_{3}\right)$ is the reciprocal of the radius of the circle passing through $z_{1}, z_{2}$ and $z_{3}$. A formula, found by Melnikov in 
$[\mathrm{Me}]$, says that

$$
c\left(z_{1}, z_{2}, z_{3}\right)^{2}=\sum_{\sigma} \frac{1}{\left(z_{\sigma(1)}-z_{\sigma(3)}\right) \overline{\left(z_{\sigma(2)}-z_{\sigma(3)}\right)}},
$$

where the summation is over all permutations of $\{1,2,3\}$. From this it is not difficult to show, see $[\mathrm{MV}]$ or $[\mathrm{MMV}]$, that if $\nu$ is a (positive) Borel measure on $\mathbb{C}$ with compact support such that

$$
\nu(B(x, r)) \leq r, \quad \text { for } x \in \mathbb{C} \text { and } r>0,
$$

then

$$
\sup _{\varepsilon>0} \int\left|\int_{\mathbb{C} \backslash B(x, \varepsilon)} \frac{d \nu(y)}{x-y}\right|^{2} d \nu(x)<\infty
$$

if and only if

$$
\iiint c(x, y, z)^{2} d \nu(x) d \nu(y) d \nu(z)<\infty
$$

On the other hand, it follows from a result of David and Léger, see [L], that if $\nu$ satisfies (1.5) and there is a Borel set $F \subset E$ such that $\mathcal{H}^{1}(F \cap B) \leq \nu(B) \leq C \mathcal{H}^{1}(F \cap B)<\infty$ for $B \subset F$, then there are rectifiable curves $\Gamma_{1}, \Gamma_{2}, \ldots$ such that

$$
\nu\left(F \backslash \bigcup_{i} \Gamma_{i}\right)=0 .
$$

The proof of Theorem 1.2 will be finished if we can use the nonremovability of $E$ to find $\nu$ satisfying (1.3) and (1.4) and $F$ as above such that $\nu(F)>0$. Remember that we only have to show that if $E$ is not removable, then $H^{1}(E \cap \Gamma)>0$ for some rectifiable curve $\Gamma$. But (1.6) and $\nu(F)>0$ give that $\nu\left(F \cap \Gamma_{i}\right)>0$ for some $i$ while (1.3) gives that $\nu(B) \leq H^{1}(B)$ for all $B \subset F$.

To find such a $\nu$ we proceed as follows. Suppose $E$ is not removable for Lipschitz harmonic functions. Then there is a non-constant Lipschitz function $u: \mathbb{C} \longrightarrow \mathbb{R}$ which is harmonic in $\mathbb{C} \backslash E$ such that $\langle\Delta u, 1\rangle \neq 0$ and $\nabla u(\infty)=0$, see [MP, Proposition 2.2]. Such a function $u$ has a representation, see, for example, [MP, Lemma 5.3],

$$
u(x)=\int \log |x-y| d \sigma(y), \quad \text { for } x \in \mathbb{C} \backslash E,
$$


where $\sigma$ is a signed Borel measure with support in $E$. Hence

$$
\nabla u(x)=\int \frac{x-y}{|x-y|^{2}} d \sigma(y), \quad \text { for } x \in \mathbb{C} \backslash E .
$$

Moreover, one can show, see [MP, Lemma 5.3], that $\sigma$ is absolutely continuous with respect to $H^{1}$ with bounded Radon-Nikodym derivative $f$. Since $u$ is Lipschitz, $\nabla u$ is bounded. Thus

$$
\int_{E} \frac{x-y}{|x-y|^{2}} f(y) d H^{1}(y)
$$

is bounded in $\mathbb{C} \backslash E$. This is the same as to say that the Cauchy transform

$$
T f(x)=\int_{E} \frac{1}{x-y} f(y) d H^{1}(y)
$$

is bounded in $\mathbb{C} \backslash E$.

Moreover, $\int_{E} f d H^{1}=c\langle\Delta u, 1\rangle \neq 0$, and we may assume that $\int_{E} f d H^{1}>0$. Almost all of the paper then consists of a modification of the measure $f d H^{1} \mid E$ to a desired measure $\nu$. The construction of $\nu$ will be given in Section 4 and the proof of (1.4) will be completed in Section 5. The modification uses stopping time arguments somewhat similar to those in [C2]. For that we need a system of dyadic cubes in $E$ with some good properties. They will be constructed in Section 3. Earlier similar systems were built in [D1], see also [D2], on regular subsets of $\mathbb{R}^{n}$ and in [C2] on spaces of homogeneous type, that is, when the measure is doubling. But now we do not have any doubling property which causes considerable complications in our applications to the Cauchy transform. The construction of the cubes will be done for an arbitrary locally finite Borel measure in $\mathbb{R}^{n}$.

In our modification result, which is stated in Section 2, we shall consider complex valued functions $f$. Then we do not get a positive measure, but we get a complex measure $g d \nu$ where $\nu$ is as above and $g$ is bounded and accretive in the sense that $\operatorname{Re} g \geq c>0$. This does not require much more work and turns out to be quite useful for Theorem 1.1.

The main obstacle for extending Theorem 1.2 to higher dimensions is the lack of the curvature method. The associated kernel in $\mathbb{R}^{n}$ is $|x|^{-n} x$. We can still form the sum of permutations as above using inner products, but when $n \geq 3$ the resulting function in $\mathbb{R}^{n} \times \mathbb{R}^{n} \times \mathbb{R}^{n}$ takes both positive and negative values which seems to make it useless. 
Some results and examples on this question in $\mathbb{R}^{n}$ can be found in $[\mathrm{U} 1]$, [U2] and $[\mathrm{MP}]$. See also $[\mathrm{F}]$ for a negative result concerning curvature in $\mathbb{R}^{n}$.

Let us also mention that at the time this introduction is revised, there is another, very nice proof of Theorems 1.1 and 1.2, see [NTV].

\section{Statement of the main technical proposition.}

We are given a compact set $E \subset \mathbb{C}$ such that

$$
0<H^{1}(E)<+\infty
$$

and a bounded $H^{1}$-measurable complex valued function $f$ on $E$, with the following property. Denote by $\mu$ the restriction of $H^{1}$ to $E$ (i.e., the measure on $\mathbb{C}$ defined by $\mu(A)=H^{1}(A \cap E)$ for all Borel sets $\left.A \subset \mathbb{C}\right)$. We assume that

$$
\|f\|_{\infty} \leq 1 \quad \text { and } \quad \int_{E} f d \mu=a>0
$$

and that the Cauchy integral of $f d \mu$, defined on $C \backslash E$ by

$$
T(f d \mu)(x)=\int_{E} \frac{f(y) d \mu(y)}{x-y}
$$

is bounded. Our main technical result is as follows.

Theorem 2.4. Let $E, \mu$ and $f$ be as above. Then there exist a positive Borel measure $\nu$ and a bounded Borel function $g$ such that

$$
\begin{gathered}
\nu(B(x, r)) \leq C r, \quad \text { for all } x \in \mathbb{C} \text { and } r>0 \\
|g(x)| \leq C \quad \text { and } \quad \operatorname{Re} g(x) \geq C^{-1}, \quad \text { for all } x \in \mathbb{C}, \\
\int g d \nu=\int f d \mu=a, \\
\left\{\begin{array}{l}
\text { there is a Borel set } F \subset E \text { such that } \\
C^{-1} \mu \leq \nu \leq \mu \text { on } F \text { and } \nu(F) \geq \frac{a}{2}
\end{array}\right.
\end{gathered}
$$


and

$$
\int T^{*}(g d \nu)^{2} d \nu<+\infty
$$

where $T^{*}$ is the maximal Cauchy operator defined by

$$
T^{*}(g d \nu)(x)=\sup _{\varepsilon>0}\left|\int_{\mathbb{C} \backslash B(x, \varepsilon)} \frac{g(y) d \nu(y)}{x-y}\right| .
$$

In this statement, the constant $C$ in (2.5) may depend on many things, including the rate at which the densities $r^{-1} \mu(B(x, r))$ stop being too large when $r$ gets small. If we know already that $\mu(B(x, r)) \leq$ $M r$ for some $M$ and all $x \in \mathbb{C}, r>0$, and if we normalized our statement by assuming that $\operatorname{diam} E=1$, the proof will give a constant in (2.5) which is less than $C_{1} M$, with $C_{1}$ an absolute constant. See the comment to this effect a little above Lemma 4.56 .

The constant $C$ in (2.6) and (2.8) can be taken to be at most $C_{1} a^{-1} \mu(E)$, where $C_{1}$ is an absolute constant. See the remark near the end of Section 4.2.

Our estimate for (2.9), just like (2.5), can depend somewhat wildly on $E$, but again if we assume that $\mu(B(x, r)) \leq M r$ as above, our proof will give an estimate on $\int T^{*}(g d \nu)^{2} d \nu$ that depends only on $M$, $\operatorname{diam} E, a^{-1} \mu(E)$, and $\|T(f d \mu)\|_{\infty}$.

If we start with a function $f$ which is real-valued, then we get a function $g$ which is real-valued as well, and the accretivity condition (2.6) simply says that

$$
C^{-1} \leq g(z) \leq C
$$

This is the case that we need for our application to Lipschitz harmonic functions, but the statement for complex valued functions $f$ is not much harder to get, and may be interesting as well.

Note that Theorem 1.2 follows from Theorem 2.4 and the discussion in Chapter 1. Because of (2.11), the measure $g d \nu$ satisfies (1.3) because of (2.5) and (1.4) by (2.9), while the condition on $F$ (for a multiple of $g d \nu)$ comes from $(2.8)$.

It is possible that our proof will give slightly more precise, BMOlike, properties, of $T^{*}(g d \nu)$ than $(2.9)$, but it is not too clear how to formulate this in a useful way.

The rest of this paper is a proof of Theorem 2.4. 


\section{Dyadic cubes with small boundaries associated to a measure in $\mathbb{R}^{n}$.}

In this chapter we want to construct partitions of the support of a given measure $\mu$ into analogues of the dyadic cubes in $\mathbb{R}^{n}$. We shall do the construction with very little information on the measure, even if some extra information on $\mu$ will be useful when we try to use our cubes.

Let $\mu$ be a locally finite (positive Borel) measure on $\mathbb{R}^{n}$, and let $E$ denote its support.

Let $C_{0}$ and $A$ be two large constants. For the construction and the statement of the theorem, we shall only require that

$$
C_{0}>1 \quad \text { and } \quad A>5000 C_{0},
$$

say, but our small boundary condition will only become useful if $A$ is larger than some high power of $C_{0}$. In our application to Theorem 2.4, we shall take $A \approx C_{0}^{100}$ and $C_{0}$ very large, for instance.

Theorem 3.2. Let $\mu, E, C_{0}$ and $A$ be as above. Then there exists a sequence of partitions of $E$ into Borel subsets $Q, Q \in \Delta_{k}$, with the following properties.

For each integer $k \geq 0$,

$$
E \text { is the disjoint union of the "cubes" } Q, Q \in \Delta_{k} \text {, }
$$

and

$$
\begin{aligned}
& \text { if } k<l, Q \in \Delta_{k} \text { and } R \in \Delta_{l}, \\
& \text { then either } Q \cap R=\varnothing \text { or else } R \subset Q \text {. }
\end{aligned}
$$

The general position of the cubes $Q$ can be described as follows. For each $k \geq 0$ and each cube $Q \in \Delta_{k}$, there is a ball

$$
B(Q)=B(x(Q), r(Q))
$$

such that

$$
\begin{gathered}
x(Q) \in E, \\
A^{-k} \leq r(Q) \leq C_{0} A^{-k}, \\
E \cap B(Q) \subset Q \subset E \cap 28 B(Q)=E \cap B(x(Q), 28 r(Q)),
\end{gathered}
$$


and

$$
\text { the balls } 5 B(Q), Q \in \Delta_{k} \text {, are disjoint. }
$$

Also, the cubes $Q \in \Delta_{k}$ have "small boundaries". For each cube $Q \in \Delta_{k}$ and each integer $l \geq 0$, set

$$
\begin{aligned}
& N_{l}^{\text {ext }}(Q)=\left\{x \in E \backslash Q: \operatorname{dist}(x, Q)<A^{-k-l}\right\}, \\
& N_{l}^{\text {int }}(Q)=\left\{x \in Q: \operatorname{dist}(x, E \backslash Q)<A^{-k-l}\right\},
\end{aligned}
$$

and

$$
N_{l}(Q)=N_{l}^{\mathrm{ext}}(Q) \cup N_{l}^{\mathrm{int}}(Q)
$$

Then

$$
\mu\left(N_{l}(Q)\right) \leq\left(C^{-1} C_{0}^{-3 n-1} A\right)^{-l} \mu(90 B(Q)) .
$$

Finally let $\mathcal{G}_{k}$ denote the set of good cubes $Q \in \Delta_{k}$ for which

$$
\mu(100 B(Q)) \leq C_{0} \mu(B(Q))
$$

and set $\mathcal{B}_{k}=\Delta_{k} \backslash \mathcal{G}_{k}$. We have that

$$
r(Q)=A^{-k}, \quad \text { when } Q \in \mathcal{B}_{k}
$$

and also

$$
\left\{\begin{array}{l}
\mu(100 B(Q)) \leq C_{0}^{-l} \mu\left(100^{l+1} B(Q)\right) \text { for all } \\
l \geq 1 \text { such that } 100^{l} \leq C_{0} \text { when } Q \in \mathcal{B}_{k}
\end{array}\right.
$$

This completes the statement of our theorem. The constant $C$ in (3.13) may depend on $n$, but nothing else. Of course the constants 28, 90, 100 and $3 n+1$ are not optimal.

The last condition (3.16) will be useful. When $Q \in \mathcal{B}_{k}$, it is not so easy to use (3.13), and we cannot really prevent situations where $Q$ is a cube with very small mass, very close to other cubes with very large masses. In these situations it is hard to get estimates on $N_{l}(Q)$ that depend on $\mu(Q)$ alone, and we shall fight this back by saying that $\mu(Q)$ 
was actually very tiny. (The constant $C_{0}^{-l}$, where $100^{l} \simeq C_{0}$, is much smaller than any given negative power of $C_{0}$ when $C_{0}$ is large.)

Now we can start the construction of cubes. The rest of this chapter will not interfere with the other parts of the paper.

Let $\mu, E, A$, and $C_{0}$ be given, as in the statement. We start our construction with an approximation to $\Delta_{0}$ (i.e., we shall first work at scale 1). A somewhat similar construction of "cubes of one generation" has been given by O'Neil in [O]. We first associate a ball $B(x)=B(x, r(x))$ to each $x \in E$, as follows.

Denote by $\mathcal{G}$ the good set of points $x \in E$ such that we can find a radius $r(x)$ such that

$$
1 \leq r(x) \leq C_{0}
$$

and

$$
\mu(B(x, 100 r(x))) \leq C_{0} \mu(B(x, r(x)))
$$

For each $x \in \mathcal{G}$, choose $r(x)$ as in (3.17), (3.18), and set $B(x)=$ $B(x, r(x))$. Set $\mathcal{B}=E \backslash \mathcal{G}$, and simply take

$$
r(x)=1 \quad \text { and } \quad B(x)=B(x, 1), \quad \text { for } x \in \mathcal{B} .
$$

By the definition of $\mathcal{G}$, we have that

$$
\begin{aligned}
& \mu\left(100^{l} B(x)\right) \leq C_{0}^{-1} \mu\left(100^{l+1} B(x)\right), \\
& \text { for all } l \geq 0 \text { such that } 100^{l} \leq C_{0},
\end{aligned}
$$

when $x \in \mathcal{B}$.

Next we want to choose, for each $x \in E$, two auxiliary radii $r_{1}(x)$ and $r_{2}(x)$ such that

$$
\begin{aligned}
& \frac{11}{10} r(x)<r_{1}(x)<\frac{12}{10} r(x), \\
& 25 r(x)<r_{2}(x)<26 r(x),
\end{aligned}
$$

and that we have the first "small boundary conditions"

$$
\mu\left(\left\{z \in \mathbb{R}^{n}:|| z-x\left|-r_{1}(x)\right| \leq \tau r(x)\right\}\right) \leq C \tau \mu\left(\frac{13}{10} B(x)\right)
$$


for $0<\tau<1 / 10$, and

$$
\mu\left(\left\{z \in \mathbb{R}^{n}:|| z-x\left|-r_{2}(x)\right| \leq \tau r(x)\right\}\right) \leq C \tau \mu(27 B(x)) .
$$

for $0<\tau<1$.

The existence of $r_{1}(x)$ and $r_{2}(x)$ is easy to derive from the standard weak- $L^{1}$ estimate for the maximal function of the measure on $\mathcal{R}_{+}$which is the image under the radial projection $z \longrightarrow|x-z|$ of $\mu_{\mid(13 / 10) B(x)}$ or $\mu_{\mid 27 B(x)}$. Here the constant $C$ does not even depend on $n$.

Choose $r_{i}(x), i=1,2$, as above, and set $B_{i}(x)=B\left(x, r_{i}(x)\right)$. Next we use the standard "Vitali-type" covering lemma (as in [M, Theorem 2.1] or see the proof on [S, p. 9]) to select a (discrete) subset $I$ of E such that

$$
\text { the balls } 5 B(x), x \in I \text {, are disjoint }
$$

and

$$
E \subset \bigcup_{x \in I} 25 B(x)
$$

(so that the $B_{2}(x), x \in I$, also cover $E$ ).

For each $x \in I$, denote by $J(x)$ the set of points $y \in I \backslash\{x\}$ such that $B_{1}(y)$ meets $B_{2}(x)$. Because (3.17) holds for all $y \in I,(3.25)$ yields that

$$
J(x) \text { has less than } C C_{0}^{n} \text { elements. }
$$

Set

$$
B_{3}(x)=B_{2}(x) \backslash\left(\bigcup_{y \in I \backslash\{x\}} B_{1}(y)\right)=B_{2}(x) \backslash\left(\bigcup_{y \in J(x)} B_{1}(y)\right) .
$$

Let us check that

$$
\frac{13}{10} B(y) \subset 90 B(x), \quad \text { for all } y \in J(x) .
$$

If $y \in J(x)$, then $(13 / 10) B(y)$ meets $26 B(x)$ because it meets $B_{2}(x)$, and so $|x-y| \leq 26 r(x)+(13 / 10) r(y)$. If (3.29) did not hold for $y$, we would also have that $|x-y|>90 r(x)-(13 / 10) r(y)$, and so $(26 / 10) r(y)>64 r(x)$. In particular, $r(y)>20 r(x),|x-y| \leq 26 r(x)+$ 
$(13 / 10) r(y)<3 r(y), x$ lies on $5 B(y)$, and this contradicts (3.25). This proves $(3.29)$.

Hence we may apply (3.24) to $x$ and (3.23) to each $y \in J(x)$ to get that

$$
\mu\left(\left\{z \in \mathbb{R}^{n}: \operatorname{dist}\left(z, \partial B_{3}(x)\right) \leq \tau\right\}\right) \leq C C_{0}^{n} \tau \mu(90 B(x))
$$

for all $x \in I$ and $0<\tau<1 / 10$. (Of course this uses (3.27), and the fact that you cannot be close to $\partial B_{3}(x)$ without being close to $\partial B_{2}(x)$ or some $\partial B_{1}(y), y \in J(x)$.)

Next put an order on $I$ such that

$$
y<x \text { in } I \text { whenever } \mu(90 B(y))<\mu(90 B(x)) .
$$

(When $\mu(90 B(x))=\mu(90 B(y))$, we can decide whether $x<y$ or not at random.) We define new sets $B_{4}(x), x \in I$, by

$$
B_{4}(x)=B_{3}(x) \backslash\left(\bigcup_{\substack{y \in I \\ y<x}} B_{3}(y)\right) .
$$

Here again, the union looks infinite, but we are only interested in the $y \in I$ such that $B_{3}(y)$ meets $B_{3}(x)$, and there are at most $C C_{0}^{n}$ of those.

Lemma 3.33. The sets $B_{4}(x), x \in I$, are disjoint and cover $E$.

This is easy. The $B_{4}(x), x \in I$, are disjoint because of the formula (3.32) alone. To prove the rest, first observe that

$$
5 B(x) \subset B_{3}(x), \quad \text { for all } x \in I .
$$

This is because $5 B(x) \subset B_{2}(x)$ by the definition of $r_{2}(x)$, and $5 B(x)$ does not meet any of the $B_{1}(y), y \neq x$, because it does not even meet the larger balls $5 B(y)$ (by (3.25)).

Because of (3.34),

$$
\text { the sets } B_{3}(x), x \in I \text {, cover } E \text {. }
$$

Indeed, if $z \in E$, then $z \in 25 B(x)$ for some $x \in I$ (by (3.26)), and if it does not lie in the corresponding $B_{3}(x)$, it must be in $B_{1}(y)$ for some $y \in J(x)$; (3.34) then says that $z \in B_{3}(y)$. 
The rest of Lemma 3.33 follows easily from (3.35) and the formula (3.32).

Once again, the set $J^{\prime}(x)$ of all $y \in I$ such that $y<x$ and $B_{3}(y)$ meets $B_{3}(x)$ has at most $C C_{0}^{n}$ elements. We may apply (3.30) to $x$ and each $y \in J^{\prime}(x)$, and we get that

$$
\mu\left(\left\{z \in \mathbb{R}^{n}: \operatorname{dist}\left(z, \partial B_{4}(x)\right) \leq \tau\right\}\right) \leq C C_{0}^{2 n} \tau \mu(90 B(x)),
$$

for all $x \in I$ and $0<\tau<1 / 10$. (We are also using the definition of $<$ on $I$.)

Notice that for each $x \in I, B_{1}(x) \subset B_{3}(x)$ (for instance by (3.34)), and since $B_{1}(x)$ does not meet any of the $B_{3}(y), y \neq x$ (by the definition (3.28) of $\left.B_{3}(y)\right)$, it is also contained in $B_{4}(x)$. Thus

$$
\frac{11}{10} B(x) \subset B_{1}(x) \subset B_{4}(x) \subset 26 B(x)
$$

(because of (3.21) and (3.22).

The sets $E \cap B_{4}(x), x \in I$, are our first approximations to the cubes $Q, Q \in \Delta_{0}$. Now we want to do a similar construction at each scale $A^{-k}, k \geq 0$, and then modify our sets so that the nesting property (3.4) holds.

Let us first apply the preceding construction at each scale $A^{-k}$. For each $k \geq 0$, we get a set $I_{k} \subset E$ of centers and, for each $x \in I_{k}$, a ball $B(x)=B^{k}(x)$ (if we want to be explicit) and a set $B_{4}(x)=B_{4}^{k}(x)$ with the following properties:

(3.41) the sets $B_{4}(x), x \in I_{k}$, cover $E$ and are disjoint,

$$
\begin{aligned}
& B(x) \text { is centered on } x, \text { and has a radius } \\
& r(x) \in\left[A^{-k}, C_{0} A^{-k}\right]
\end{aligned}
$$

the balls $5 B(x), x \in I_{k}$, are disjoint,

$$
\frac{11}{10} B(x) \subset B_{4}(x) \subset 26 B(x),
$$

$$
\begin{aligned}
\mu\left(\left\{z \in \mathbb{R}^{n}: \operatorname{dist}\left(z, \partial B_{4}(x)\right)\right.\right. & \left.\left.\leq \tau A^{-k}\right\}\right) \\
& \leq C C_{0}^{2 n} \tau \mu(90 B(x)),
\end{aligned}
$$

for all $k \geq 0$, all $x \in I_{k}$, and $0<\tau<1 / 10$. 
Moreover, if $x \in I_{k}$, then either

$$
\mu(100 B(x)) \leq C_{0} \mu(B(x)),
$$

or else $r(x)=A^{-k}$ and

$$
\mu\left(100^{l} B(x)\right) \leq C_{0}^{-1} \mu\left(100^{l+1} B(x)\right)
$$

for all $l \geq 0$ such that $100^{l} \leq C_{0}$.

Next we want to replace each set $B_{4}(x)=B_{4}^{k}(x), x \in I_{k}$, by finer versions obtained by taking unions of sets $B_{4}(y)$ from smaller generations. We have to do this in a coherent way.

For each $k \geq 1$ and $y \in I_{k}$, let $h(y)$ denote the point $x \in I_{k-1}$ such that $y \in B_{4}^{k-1}(x)$. There is nothing very special about this choice; any $x$ such that $E \cap B_{4}^{k-1}(x) \cap B_{4}^{k}(y) \neq \varnothing$, for instance, would work as well. It will be just as convenient to choose $h(y)$ so that

$$
y \in B_{4}^{k-1}(h(y)),
$$

though.

Also set $h^{l}(y)=h \circ \cdots \circ h(y)(l$ times $)$ when $k \geq l$. Thus $h^{l}(y) \in$ $I_{k-l}$. Define, for each $x \in I_{k}$, new sets $D_{l}^{k}(x), l \geq 0$, by

$$
D_{l}^{k}(x)=\bigcup_{\substack{y \in I_{k+l} \\ h^{l}(y)=x}} B_{4}^{k+l}(y) .
$$

(In particular, use the convention that $D_{0}^{k}(x)=B_{4}(x)$.) Our intention is to take the limits of the $D_{l}^{k}(x)$, with $k$ and $x$ fixed and $l \longrightarrow+\infty$, as our almost final version of $\Delta_{k}$.

For $k$ and $l$ fixed, the sets $h^{-l}(x), x \in I_{k}$, form a partition of $I_{k+l}$, and hence

$$
\text { the sets } D_{l}^{k}(x), x \in I_{k} \text {, are disjoint and cover } E \text {, }
$$

because of the corresponding properties (3.41) of the sets $B_{4}^{k+l}(y), y \in$ $I_{k+l}$.

Lemma 3.48. We have that

$$
\operatorname{dist}\left(z, E \cap D_{l}^{k}(x)\right) \leq 26 C_{0} A^{-k-l-1},
$$


for all $z \in D_{l+1}^{k}(x)$ (and all choices of $k \geq 0, x \in I_{k}$, and $l \geq 0$ ).

Indeed, let $z \in D_{l+1}^{k}(x)$ be given. By definitions, there is an $y \in$ $I_{k+l+1}$ such that $h^{l+1}(y)=x$ and $z \in B_{4}^{k+l+1}(y)$. Then $|z-y| \leq$ $26 r(y) \leq 26 C_{0} A^{-k-l-1}$ by (3.40) and (3.38). On the other hand, $y \in B_{4}^{k+l}(h(y))$ by the definition of $h$ (see (3.45)), and so $y \in D_{l}^{k}(x)$ because $h^{l}(h(y))=x$. This proves the lemma.

Since $A \geq 10$, say, by (3.1), we can iterate the estimate from Lemma 3.48 and get that

$$
\operatorname{dist}\left(z, E \cap D_{l_{0}}^{k}(x)\right) \leq 50 C_{0} A^{-k-l_{0}-1},
$$

for all $z \in \bigcup_{l \geq l_{0}} D_{l}^{k}(x)$ and all $l_{0} \geq 0$. (Connect $z$ to $D_{l_{0}}^{k}(x)$ by a sequence of points in the $D_{m}^{k}(x), l_{0}<m<l$, and sum a geometric series.)

Let us now prove an estimate in the other direction. We claim that

$$
\operatorname{dist}\left(z, E \cap D_{l+1}^{k}(x)\right) \leq 26 C_{0} A^{-k-l}, \quad \text { for all } z \in D_{l}^{k}(x) .
$$

Remember that $D_{l}^{k}(x)$ is the union of the $B_{4}^{k+l}(y), y \in h^{-l}(x)$. Thus, by (3.38) and (3.40), it will be enough to show that each $y \in$ $h^{-l}(x)$ lies on $D_{l+1}^{k}(x)$. Let $u \in I_{k+l+1}$ be such that $y \in B_{4}^{k+l+1}(u)$. It is enough to show that $h^{l+1}(u)=x$, or even that $h(u)=y$ (because we already know that $\left.h^{l}(y)=x\right)$. Since $y \in B_{4}^{k+l+1}(u)$, we know that $|y-u| \leq 26 C_{0} A^{-k-l-1}$. Since by (3.1) $A>26 C_{0}$, this implies that $u \in B(y)$ (by (3.38)), and then that $u \in B_{4}(y)$ (by (3.40)) and $h(u)=y$ (by the definition of $h(u)$, see (3.45)). This proves our claim (3.51).

From Lemma 3.48 and (3.51) we deduce that the Hausdorff distance between the sets $D_{l}^{k}(x)$ and $D_{l+1}^{k}(x)$ is at most $26 C_{0} A^{-k-l}$. Thus the closures of these sets converge (for the Hausdorff metric on compact sets) to a set

$$
D(x)=D^{k}(x)=\lim _{l \rightarrow+\infty} \overline{D_{l}^{k}(x)} .
$$

The sets $D(x), x \in I_{k}$, are almost our cubes $Q, Q \in \Delta_{k}$, but we may have lost the property that they are disjoint, and so we want to fix that. Before, let us collect a few of the properties of the $D^{k}(x)^{\prime}$ 's. Let us first check that

$$
D^{k}(x) \subset E \cap 28 B(x), \quad \text { for all } x \in I_{k} .
$$


Indeed, let $z \in D^{k}(x)$ be given. Then $z=\lim _{l \rightarrow+\infty} z_{l}$ for some sequence $\left\{z_{l}\right\}$ such that $z_{l} \in D_{l}^{k}(x)$. Because of Lemma 3.48 (or (3.51)), $\operatorname{dist}\left(z_{l}, E\right) \leq 26 C_{0} A^{-k-l}$, and so $z \in E$. From (3.40), the fact that $D_{0}^{k}(x)=B_{4}^{k}(x)$ and (3.50) (with $l_{0}=0$ ), we deduce that $\left|x-z_{l}\right| \leq$ $26 r(x)+50 C_{0} A^{-k-1}$ for all $l$; (3.53) follows because $A>50 C_{0}$.

Next we check that

$$
E=\bigcup_{x \in I_{k}} D^{k}(x)
$$

We already know that $D^{k}(x) \subset E$. If $z \in E$, then for each $l$ there is an $x_{l} \in I_{k}$ such that $z \in D_{l}^{k}\left(x_{l}\right)$ (because of (3.47)). Moreover, there is only a finite set of points $x \in I_{k}$ for which $28 B(x)$ contains $z$ (because the $5 B(x)$ are disjoint and have comparable diameters). Since (3.53) tells us that all the $x_{l}$ 's must be picked in this finite set, we see that there is an $x \in I_{k}$ such that $z \in D_{l}^{k}(x)$ for infinitely many values of $l$. Then $z \in D^{k}(x)$, and (3.54) holds.

Lemma 3.55. For each $x \in I_{k}, E \cap B(x) \subset D^{k}(x)$, and $E \cap B(x)$ does not meet any of the $D^{k}\left(x^{\prime}\right), x^{\prime} \in I_{k} \backslash\{x\}$.

Because we already know that the $D^{k}\left(x^{\prime}\right), x^{\prime} \in I_{k}$, cover $E$, it is enough to prove the second half of the statement. Let $z \in E \cap B(x)$ be given, and suppose that $z \in D^{k}\left(x^{\prime}\right)$ for some $x^{\prime} \in I_{k}$. Then we can find a point $z_{l}$ such that $z_{l} \in D_{l}^{k}\left(x^{\prime}\right)$ for some $l>1$ and $\left|z-z_{l}\right|<r(x) / 100$, say. From (3.50) (with $l_{0}=0$ ) we deduce that dist $\left(z_{l}, E \cap B_{4}^{k}\left(x^{\prime}\right)\right) \leq$ $50 C_{0} A^{-k-1}$. Since $A>5000 C_{0}$ by (3.1), we get that $\operatorname{dist}(z, E \cap$ $\left.B_{4}^{k}\left(x^{\prime}\right)\right) \leq r(x) / 50$. Then $E \cap B_{4}^{k}\left(x^{\prime}\right)$ meets $(11 / 10) B(x)$, and since (3.40) and (3.41) tell us that $B_{4}^{k}\left(x^{\prime}\right)$ is disjoint from $(11 / 10) B(x)$ when $x^{\prime} \neq x$, we get that $x^{\prime}=x$. This proves the lemma.

Lemma 3.56. For each $x \in I_{k}$ and each $l \geq 0$,

$$
D^{k}(x)=\bigcup_{\substack{y \in I_{k+l} \\ h^{l}(y)=x}} D^{k+l}(y) .
$$


Indeed for each $m \geq 0$, by (3.46),

$$
\begin{aligned}
D_{m+l}^{k}(x) & =\bigcup_{z \in h^{-m-l}(x)} B_{4}^{k+m+l}(z) \\
& =\bigcup_{y \in h^{-l}(x)}\left(\bigcup_{z \in h^{-m}(y)} B_{4}^{k+m+l}(z)\right) \\
& =\bigcup_{y \in h^{-l}(x)} D_{m}^{k+l}(y)
\end{aligned}
$$

The lemma follows by taking closures in (3.57), and then passing to the limit.

Now we want to modify our sets $D^{k}(x)$ to make them disjoint. We need to be a little careful because we want some coherence between the different values of $k$, but nothing too interesting will be happening here. Our future estimates will show that the various intersections of the $D^{k}(x), x \in I_{k}$, have measure 0 , so we do not really need to worry about the effect of our choices now on singular integrals, for instance.

First put any order on $I_{0}$. This does not need to be the same one as in the definition of $B_{3}(x)$, but we really do not care. Then put orders on $I_{1}$, then $I_{2}$, then $I_{3}$, etc., with the only constraint that

$$
y_{1}<y_{2} \text { on } I_{k+1} \text { whenever } h\left(y_{1}\right)<h\left(y_{2}\right) \text { on } I_{k} \text {. }
$$

In other words, when we define the order on $I_{k+1}$, we decompose $I_{k+1}$ into the classes $h^{-1}(x), x \in I_{k}$, and make sure that they come out in the same order on $I_{k+1}$ as the $x \in I_{k}$. The order in each class is irrelevant.

For each $k \geq 0$ and each $x \in I_{k}$, set

$$
Q(x)=Q^{k}(x)=D^{k}(x) \backslash\left(\bigcup_{\substack{y \in I_{k} \\ y<x}} D^{k}(y)\right)
$$

These are the cubes we wanted. To get back to the notations of Theorem 3.1, we set $\Delta_{k}=\left\{Q^{k}(x), x \in I_{k}\right\}$. We should now start verifying the various conclusions of Theorem 3.2.

The fact that for each $k \geq 0, E$ is the disjoint union of the $Q^{k}(x)$, $x \in I_{k}$, is an easy consequence of (3.54) and (3.59). This takes care of (3.3). 
Let us check now that if $x \in I_{k}$ and $y \in I_{k+1}$ is such that $h(y)=x$, then $Q(y) \subset Q(x)$. We already know from Lemma 3.56 that $Q(y) \subset$ $D^{k+1}(y) \subset D^{k}(x)$, so it is enough to check that $Q(y)$ does not meet any of the $D^{k}\left(x^{\prime}\right), x^{\prime}<x$ (the sets that were removed from $D^{k}(x)$ to get $\left.Q^{k}(x)\right)$. If it $\operatorname{did}$, then $Q(y)$ would also meet $D^{k+1}\left(y^{\prime}\right)$ for some $y^{\prime} \in I_{k+1}$ such that $h\left(y^{\prime}\right)=x^{\prime}$ (because of Lemma 3.56). This is not possible, because $y^{\prime}<y$ (since $x^{\prime}<x$ ). Thus $Q(y) \subset Q(x)$, as promised.

Now if $y \in I_{k+1}$ is such that $h(y) \neq x$, then $Q(y) \subset Q(h(y))$, and so $Q(y)$ does not meet $Q(x)$ (by (3.3)). This implies the nesting property (3.4). We even have that $Q^{k+l}(y) \subset Q^{k}(x)$ if and only if $h^{l}(y)=x$.

The properties (3.5), (3.6) and (3.7) are just the same as (3.38) (if we set $B(Q(x))=B(x)$ for $x \in I_{k}$ ), while (3.8) follows from (3.53) and Lemma 3.55. The balls $5 B(Q), Q \in \Delta_{k}$ are indeed disjoint (as needed for (3.9)) by (3.39). The small boundary property (3.10-13) will be more interesting.

Fix $x \in I_{k}$, let $Q=Q(x)$ and let $N_{l}(Q)$ be as in (3.10-12). Also denote by $\mathcal{A}_{l}(Q)$ the set of the cubes $R \in \Delta_{k+l}$ such that $N_{l}(Q) \cap R \neq \varnothing$. We first consider the case $l=1$.

\section{Lemma 3.60.}

$$
\sum_{S \in \mathcal{A}_{1}(Q)} \mu(90 B(S)) \leq C C_{0}^{3 n+1} A^{-1} \mu(90 B(Q)) .
$$

First we want to check that

$$
\operatorname{dist}\left(z, \partial B_{4}(x)\right) \leq 51 C_{0} A^{-k-1}, \quad \text { for all } z \in N_{1}(Q) \text {. }
$$

Let $z \in N_{1}(Q)$ be given. Then there are points $z_{1}$ and $z_{2}$, with $z_{1} \in$ $Q(x)$ and $z_{2} \in E \backslash Q(x)$, such that $z=z_{1}$ or $z_{2}$ and $\left|z_{1}-z_{2}\right|<A^{-k-1}$. Let $x_{2} \in I_{k}$ be such that $z_{2} \in Q\left(x_{2}\right)$.

Since $z_{1} \in Q(x) \subset D^{k}(x)$, we can find points of $\bigcup_{l \geq 0} D_{l}^{k}(x)$ that are as close as we want to $z_{1}$. Because of (3.50), applied with $l_{0}=0$, those points are all within $50 C_{0} A^{-k-1}$ of $E \cap D_{0}^{k}(x)=E \cap B_{4}(x)$. So $\operatorname{dist}\left(z_{1}, B_{4}(x)\right) \leq 50 C_{0} A^{-k-1}$. The same estimate with $z_{2}$ gives that $\operatorname{dist}\left(z_{2}, B_{4}\left(x_{2}\right)\right) \leq 50 C_{0} A^{-k-1}$, and since $B_{4}\left(x_{2}\right)$ does not meet $B_{4}(x)$ (by $(3.41)$ ), we get (3.61).

Now we can prove Lemma 3.60. Each $90 B(S), S \in \mathcal{A}_{1}(Q)$, meets $N_{1}(Q)$ by (3.8). Therefore their union lies entirely within $300 C_{0} A^{-k-1}$ of $\partial B_{4}(x)$ by $(3.61)$. By (3.42) this union has a measure at most 
$C C_{0}^{2 n}\left(C_{0} A^{-1}\right) \mu(90 B(x))$. (Here we have applied (3.42) with $\tau=$ $300 C_{0} A^{-1}$; we have that $\tau<1 / 10$ because of (3.1).) Also, a given point of the union cannot lie in more than $C C_{0}^{n}$ balls $90 B(S)$, because all these balls have centers at distances greater than $A^{-k-1}$ from each other, and at the same time at most $90 C_{0} A^{-k-1}$ from the given point. The lemma follows from all this. This proves also (3.13) for $l=1$.

Now we want to estimate $\mu\left(N_{l}(Q)\right)$ for $l>1$. (When $l=0,(3.13)$ follows immediately from the fact that $N_{0} \subset 90 B(Q)$, by (3.8).) We shall prove the estimate

$$
\sum_{S \in \mathcal{A}_{l}(Q)} \mu(90 B(S)) \leq\left(C C_{0}^{3 n+1} A^{-1}\right)^{l} \mu(90 B(Q)),
$$

which clearly is stronger than (3.13).

Let $S \in \mathcal{A}_{l}(Q)$ and choose $z \in S \cap N_{l}(Q)$. Then there are $z_{1}$ and $z_{2}$ such that $z=z_{1}$ or $z_{2}, z_{1} \in Q, z_{2} \in Q^{\prime}$ for some $Q^{\prime} \in \Delta_{k}, Q^{\prime} \neq Q$, and $\left|z_{1}-z_{2}\right|<A^{-k-l}$. Clearly, $z_{1}, z_{2} \in N_{l}(Q)$. Let $R$ be the cube of $\Delta_{k+l-1}$ which contains $z$. Then $R \in \mathcal{A}_{l-1}(Q)$, as $z \in N_{l}(Q) \subset N_{l-1}(Q)$, and $S \subset R$. Since $\left|z_{1}-z_{2}\right|<A^{-k-l}$, both $z_{1}$ and $z_{2}$ belong to $N_{1}(R)$ and so $S \in \mathcal{A}_{1}(R)$. This shows that

$$
\mathcal{A}_{l}(Q) \subset \bigcup_{R \in \mathcal{A}_{l-1}(Q)} \mathcal{A}_{1}(R)
$$

Because of this we can deduce from Lemma 3.60 that

$$
\begin{aligned}
\sum_{S \in \mathcal{A}_{l}(Q)} \mu(90 B(S)) & \leq \sum_{R \in \mathcal{A}_{l-1}(Q)} \sum_{S \in \mathcal{A}_{1}(R)} \mu(90 B(S)) \\
& \leq C C_{0}^{3 n+1} A^{-1} \sum_{R \in \mathcal{A}_{l-1}(Q)} \mu(90 B(R)) .
\end{aligned}
$$

Iterating this we get (3.62).

Finally (3.14-3.16) is the same as (3.43-3.44). This completes the proof of Theorem 3.1.

\section{Construction of $g d \nu$.}

Let $E, \mu$, and $f$ be given, as in the statement of Theorem 2.4. In this chapter we construct a measure $g d \nu$, and check that it satisfies the 
requirements (2.5)-(2.8) of the theorem (i.e., all the requirements that do not concern the Cauchy integral). The construction itself will only use the hypotheses (2.1) and (2.2). It is only when we are finished with the construction that we shall start thinking about Cauchy kernels, except for the detail that we shall keep the right to choose the constant $C_{0}$ large enough for the arguments of Chapter 5 to work.

\subsection{The construction itself.}

The first thing we do is to apply the construction of Chapter 3 to find collections of cubes $Q, Q \in \Delta_{k}$, as in Theorem 3.2. As was explained before, we want to keep the right to choose $C_{0}$ enormous later (just after (5.29) and before (5.148)); on the other hand we can already decide to take

$$
A=C C_{0}^{100}, \quad \text { with } C \text { as in (3.13). }
$$

Without loss of generality, we can assume that $\operatorname{diam} E=1$, and then our construction gives that $\Delta_{0}$ is composed of just one cube $Q^{0}$. We shall often use the following conventions about cubes. The generation of a cube $Q$ is the integer $k(Q)$ such that $Q \in \Delta_{k(Q)}$. Note that we shall always consider $Q$ as a subset of $E$, plus the information of its generation. Thus it could be that cubes from different generations correspond to the same set of $E$, but we shall still think of them as different cubes.

If $k(Q) \geq 1$, the parent of $Q$ is the cube $\widehat{Q}$ of $\Delta_{k(Q)-1}$ such that $Q \subset \widehat{Q}$. The brothers of $Q$ are the other cubes of $\Delta_{k(Q)}$ that are contained in $\widehat{Q}$. The children of $Q$ are the cubes of $\Delta_{k(Q)+1}$ that are contained in $Q$. (This does not require $k(Q) \geq 1$.)

For each $Q \in \Delta=\bigcup_{k \geq 0} \Delta_{k}$, set

$$
\mathcal{C}(Q)=\partial\left(\frac{1}{2} B(Q)\right)
$$

where $B(Q)$ is the ball associated to $Q$ in the statement of Theorem 3.2. This circle has the advantage that

$$
\operatorname{dist}(\mathcal{C}(Q), E \backslash Q) \geq \frac{1}{2} A^{-k(Q)}
$$

by (3.7) and (3.8). 
Our first concern will be to get rid of the places where the density of $\mu$ is too large. For each $x \in E$, set

$$
\mathcal{M}_{\mu}(x)=\sup _{r>0} r^{-1} \mu(B(x, r))=\sup _{r>0} r^{-1} H^{1}(E \cap B(x, r)) .
$$

Let $M$ be a large constant, to be chosen soon, and set

$$
\mathcal{O}(M)=\left\{x \in E: \mathcal{M}_{\mu}(x)>M\right\}
$$

Because $H^{1}(E)<\infty, \mathcal{M}_{\mu}(x)<+\infty$ for $\mu$-almost every $x \in E$ and so we can choose $M$ so large that

$$
\mu(\mathcal{O}(M))<\frac{a}{8}
$$

where $a$ is as in (2.2). With this choice of $M$ done, let us start to describe a few categories of cubes which we want to use in a stoppingtime construction.

Denote by HD (high density) the set of maximal cubes $Q \in \Delta$ that are contained in $\mathcal{O}(M)$. (When two cubes correspond to the same set of $E$, the "largest one" is by definition the one from the earliest (i.e., lowest) generation.)

Let $0<a_{0}<a_{1}<a$ be two small constants, to be chosen later (around (4.68)). Denote by MI (medium-sized integral) the set of maximal cubes $Q$ with the property that

$$
a_{0} \mu(Q)<\operatorname{Re} \int_{Q} f d \mu \leq a_{1} \mu(Q)
$$

Next denote by LI (low integral) the set of maximal cubes $Q$ with the properties that

$$
Q \text { is not strictly contained in any cube of } \mathrm{HD} \cup \mathrm{MI} \text {, }
$$

and

$$
\operatorname{Re} \int_{Q} f d \mu \leq a_{0} \mu(Q)
$$

(If $Q=Q^{\prime}$ as sets but $k\left(Q^{\prime}\right)<k(Q)$, we still say that $Q$ is strictly contained in $Q^{\prime}$.) 
Note that if $Q \in \mathrm{LI}$, then $k(Q)$ (the generation of $Q$ ) is greater or equal to 1 , since the top cube $Q^{0}=E$ does not satisfy (4.9).

Denote by PLI the set of parents of cubes in LI. We have that

$$
\operatorname{Re} \int_{Q} f d \mu>a_{1} \mu(Q), \quad \text { when } Q \in \mathrm{PLI}
$$

since we know that $Q$ is not contained in any cube of MI (because some child of $Q$ satisfies (4.8)), and also that $Q$ does not satisfy (4.9) (because it satisfies (4.8)). We shall essentially want to replace all cubes of $\mathrm{HD} \cup \mathrm{MI} \cup \mathrm{LI}$ by circles $\mathcal{C}(Q)$, but we want to get organized first.

Let $I_{1}$ denote the set of maximal cubes of HD $\cup$ MI that are not contained in any cube of LI. Thus

$$
\text { the cubes of } I_{1} \cup \text { LI are all disjoint from each other . }
$$

(The cubes of LI are disjoint by maximality and are never strictly contained in cubes of $I_{1}$, by (4.8), and the cubes of $I_{1}$ are disjoint from each other by maximality and are never contained in cubes of LI, by definition.)

REMARK 4.12. If $Q$ is any cube such that $Q \subset \mathcal{O}(M)$ or $\operatorname{Re} \int_{Q} f d \mu \leq$ $a_{1} \mu(Q)$, then $Q$ is contained in some cube of $I_{1} \cup \mathrm{LI}$.

Indeed, if $Q$ is contained in some cube of $\mathrm{HD} \cup \mathrm{MI}$, then it is either contained in a cube of $I_{1}$ or else the maximal cube of HD $\cup$ MI that contains it has been removed because it was contained in some cube of LI. In both cases $Q$ is contained in a cube of $I_{1} \cup$ LI. If $Q$ satisfies one of the properties of the remark but is not contained in any cube of $H D \cup M I$, then it satisfies (4.9) because it does not satisfy (4.7). It is contained in a maximal cube $R$ that satisfies (4.9); $R$ satisfies (4.8) as well because $Q$ already does. In this case $R$ lies in LI, and we are happy.

By our choice of $M$, recall (4.6), $E$ is not contained in $\mathcal{O}(M)$. Then the top cube $Q^{0}$ is not in $I_{1} \cup$ LI. We shall use later the fact that

$$
\left\{\begin{array}{l}
\mu(100 B(Q)) \leq C A^{-k(Q)} \text { for all the cubes of } I_{1} \cup \mathrm{LI} \text { and } \\
\text { all the cubes that are not contained in any cube of } I_{1} \cup \mathrm{LI},
\end{array}\right.
$$

which follows from the fact that if $Q$ is as in (4.13), then either $Q=$ $Q^{0}$ or else the parent of $Q$ cannot be contained in a cube of HD, by Remark 4.12. 
Next set $I=I_{1} \cup$ PLI. Note that the cubes of $I$ are not necessarily disjoint now: cubes of PLI can contain other cubes of $I$. For each cube $Q \in I$, define the "operating generation $k_{Q}$ " by

$$
\begin{cases}k_{Q}=k(Q), & \text { if } Q \in I_{1}, \\ k_{Q}=k(Q)+1, & \text { if } Q \in \mathrm{PLI} .\end{cases}
$$

We put an order on $I$ such that $Q$ comes before $Q^{\prime}$ if $k_{Q} \leq k_{Q^{\prime}}$, and also, when $k_{Q}=k_{Q^{\prime}}, Q$ comes before $Q^{\prime}$ if $Q \in$ PLI and $Q^{\prime} \in I_{1}$. (A cube cannot be in PLI and $I_{1}$ at the same time, by (4.11).) Now denote by $Q_{n}, n \geq 1$, the $n^{\text {th }}$ cube of $I$ for this order. Also set $k_{n}=k_{Q_{n}}$. Thus $k_{n+1} \geq k_{n}$, and if $k_{n+1}=k_{n}$ we cannot have that $Q_{n} \in I_{1}$ and $Q_{n+1} \in$ PLI. In what follows, we shall always act as if $I$ was infinite. If this is not the case, simply truncate the construction described below.

We want to define a sequence of measures $F_{n}$. We shall do this by induction on $n$. Each $F_{n}$ will be of the form

$$
F_{n}=\rho_{n} f d \mu+\sum_{m \leq n} \alpha_{m} d \nu_{m},
$$

where the $\alpha_{m}$ 's will be complex numbers, each $\nu_{m}$ will be a positive constant multiple of the arclength measure on some circle $\mathcal{C}_{m}$, and $\rho_{n}$ will be a Borel function on $E$ such that

$$
0 \leq \rho_{n} \leq 1 .
$$

Moreover, the function $\rho_{n}$ will be constant on each of the cubes $Q_{m}$, $m>n$.

Now let us construct the measures $F_{n}$ for good. We start with $F_{0}=f d \mu$ (i.e., no measure $\nu_{0}$ and $\rho_{0} \equiv 1$ ). Now suppose that we already know $F_{n-1}$, and that it has the form described above, and let us construct $F_{n}$.

The first case is when $Q_{n} \in I_{1}$. In this case we simply want to replace $Q_{n}$ with $\mathcal{C}_{n}=\mathcal{C}\left(Q_{n}\right)$. We want to do this without changing $\int F_{n}$, though. Denote by $\rho_{n}^{*}$ the constant value of $\rho_{n-1}$ on $Q_{n}$. We take

$$
\begin{gathered}
d \nu_{n}=\rho_{n}^{*} \frac{\mu\left(Q_{n}\right)}{H^{1}\left(\mathcal{C}_{n}\right)} d H^{1} \mid \mathcal{C}_{n}, \\
\rho_{n}=\rho_{n-1} \mathbf{1}_{E \backslash Q_{n}}, \\
\alpha_{n}=\mu\left(Q_{n}\right)^{-1} \int_{Q_{n}} f d \mu,
\end{gathered}
$$


and define $F_{n}$ by the formula (4.15). Thus

$$
F_{n}=F_{n-1}-\mathbf{1}_{Q_{n}} \rho_{n-1} f d \mu+\alpha_{n} d \nu_{n}
$$

It is easy to see that $F_{n}$ is of the type described above. In particular, if $m>n$, then $\rho_{n-1}$ was constant on $Q_{m}$ by induction hypothesis, and so it is enough to check that $Q_{n}$ does not meet $Q_{m}$. If $Q_{m} \in I_{1}$, this follows from the disjointness of the cubes of $I_{1}$ (by (4.11)). Otherwise $Q_{m} \in$ PLI, and hence $k_{m}>k_{n}$ by definition of our order. Now if $Q_{m} \cap Q_{n} \neq \varnothing$, then $Q_{m} \subset Q_{n}$, and this is not possible because $Q_{m}$ contains some cubes of LI, which are disjoint from $Q_{n}$ by (4.11).

Let us collect a few easy facts for future reference

$$
\begin{gathered}
\left\|\nu_{n}\right\|=\rho_{n}^{*} \mu\left(Q_{n}\right) \leq \mu\left(Q_{n}\right), \\
\int F_{n}=\int F_{n-1}
\end{gathered}
$$

(compare (4.17), (4.19) and (4.20));

$$
\left|\alpha_{n}\right| \leq 1
$$

(by $(2.2)$ ). Let us also check that

$$
\operatorname{Re} \alpha_{n} \geq a_{0} \text {. }
$$

If $Q_{n} \in \mathrm{MI}$, this follows from (4.7). Otherwise, we are not worried either; we can use the fact that $Q_{n} \in I_{1}$, and hence is not contained in any cube of LI. Since $Q_{n}$ is not strictly contained in any cube of $\mathrm{HD} \cup \mathrm{MI}$ (it is a maximal cube of HD $\cup \mathrm{MI}$ ), it cannot satisfy (4.9) because otherwise it would be in LI. This proves (4.24).

Now let us define $F_{n}$ when $Q_{n} \in$ PLI. Some amount of notation will be useful. Denote by $\mathcal{A}_{n}$ the set of the children of $Q_{n}$ that lie in LI. These are the cubes $Q$ such that $k(Q)=k_{n}, Q \subset Q_{n}$, and $Q \in$ LI. Also let $\mathcal{A}_{n}^{*}$ denote the set of the children of $Q_{n}$ that are not in LI. Set

$$
H_{n}=\bigcup_{Q \in \mathcal{A}_{n}} Q \quad \text { and } \quad G_{n}=\bigcup_{Q \in \mathcal{A}_{n}^{*}} Q
$$

Thus $Q_{n}$ is the disjoint union of $H_{n}$ and $G_{n}$. We want to remove $H_{n}$ and keep $G_{n}$. 
Choose any cube $Q_{n}^{*} \in \mathcal{A}_{n}$, and take $\mathcal{C}_{n}=\mathcal{C}\left(Q_{n}^{*}\right)$. Also choose

$$
d \nu_{n}=\rho_{n}^{*} \frac{\mu\left(H_{n}\right)}{H^{1}\left(\mathcal{C}_{n}\right)} d H^{1} \mid \mathcal{C}_{n},
$$

where $\rho_{n}^{*}$ still denotes the constant value of $\rho_{n-1}$ on $Q_{n}$ (which exists by induction hypothesis). We want to define $\rho_{n}$ by

$$
\rho_{n}(x)= \begin{cases}\rho_{n-1}(x), & \text { when } x \in E \backslash Q_{n}, \\ 0, & \text { when } x \in H_{n}, \\ \left(1-\theta_{n}\right) \rho_{n-1}(x), & \text { when } x \in G_{n},\end{cases}
$$

with a number $\theta_{n}$ such that

$$
\theta_{n} \operatorname{Re} \int_{G_{n}} f d \mu=a_{0} \mu\left(H_{n}\right)-\operatorname{Re} \int_{H_{n}} f d \mu
$$

Let us check that $\operatorname{Re} \int_{G_{n}} f d \mu \neq 0$, and at the same time derive some estimates on $\theta_{n}$. First observe that $H_{n}$ is a disjoint union of cubes of LI, and so (4.9) tells us that

$$
\operatorname{Re} \int_{H_{n}} f d \mu \leq a_{0} \mu\left(H_{n}\right)
$$

Also

$$
\operatorname{Re} \int_{Q_{n}} f d \mu \geq a_{1} \mu\left(Q_{n}\right)
$$

by (4.10). Then

$$
\begin{aligned}
\operatorname{Re} \int_{G_{n}} f d \mu & =\operatorname{Re} \int_{Q_{n}} f d \mu-\operatorname{Re} \int_{H_{n}} f d \mu \\
& \geq a_{1} \mu\left(Q_{n}\right)-\operatorname{Re} \int_{H_{n}} f d \mu \\
& \geq a_{1} \mu\left(Q_{n}\right)-a_{0} \mu\left(H_{n}\right) \\
& >\left(a_{1}-a_{0}\right) \mu\left(Q_{n}\right) .
\end{aligned}
$$

From this we deduce that (4.28) defines a unique $\theta_{n}$, and also that $\theta_{n}$ has the same sign as $a_{0} \mu\left(H_{n}\right)-\operatorname{Re} \int_{H_{n}} f d \mu$, i.e., is nonnegative by 
(4.29). From the second line of (4.31) we deduce that $\operatorname{Re} \int_{G_{n}} f d \mu$ is larger than the right-hand side of (4.28). Thus

$$
0 \leq \theta_{n}<1
$$

We also have the brutal estimate that

$$
a_{0} \mu\left(H_{n}\right)-\operatorname{Re} \int_{H_{n}} f d \mu \leq\left(a_{0}+1\right) \mu\left(H_{n}\right)
$$

(since $\|f\|_{\infty} \leq 1$ ), from which we deduce that

$$
\theta_{n} \leq \frac{a_{0}+1}{a_{1}-a_{0}} \frac{\mu\left(H_{n}\right)}{\mu\left(Q_{n}\right)}
$$

with the help of (4.31).

We may now return to the definition of $F_{n}$ when $Q_{n} \in$ PLI. We already defined $\nu_{n}$ and $\rho_{n}$. Now take

$$
\begin{gathered}
\operatorname{Re} \alpha_{n}=a_{0}, \\
\operatorname{Im} \alpha_{n}=\mu\left(H_{n}\right)^{-1}\left(\operatorname{Im} \int_{H_{n}} f d \mu+\theta_{n} \operatorname{Im} \int_{G_{n}} f d \mu\right)
\end{gathered}
$$

and define $F_{n}$ by

$$
F_{n}=F_{n-1}-\mathbf{1}_{H_{n}} \rho_{n-1} f d \mu-\theta_{n} \mathbf{1}_{G_{n}} \rho_{n-1} f d \mu+\alpha_{n} d \nu_{n} .
$$

Then (4.15) follows from the definition (4.27) of $\rho_{n}$ and our induction hypothesis.

Now we should check that $F_{n}$ has the form described above. First, $0 \leq \rho_{n} \leq 1$ because $0 \leq \theta_{n} \leq 1$. Let us check that $\rho_{n}$ is constant on each cube $Q_{m}, m>n$. If $k\left(Q_{m}\right) \geq k_{n}$, then this follows from the induction hypothesis, and the fact that the cubes that compose $H_{n}$ and $G_{n}$ are of generation $k_{n}$. Otherwise, $Q_{m} \in \mathrm{PLI}$ and $k_{m}=k_{n}$, because our order on $I$ is such that $k_{m} \geq k_{n}$. In this case $Q_{m}$ and $Q_{n}$ are disjoint because they are of the same generation, and $\rho_{n}=\rho_{n-1}$ or $Q_{m}$. Thus $F_{n}$ has the form required for the continuation of the induction.

This completes our definition of the sequence $F_{n}$. If $I$ is finite, we simply stop when there is no $Q_{n}$ left and adapt the rest of this proof in the obvious way. Let us also collect a few estimates from the construction of $F_{n}$ when $Q_{n} \in$ PLI. Obviously (4.26) gives

$$
\left\|\nu_{n}\right\|=\rho_{n}^{*} \mu\left(H_{n}\right) \leq \mu\left(H_{n}\right),
$$


we deduce from $(4.34),(4.35),(4.33)$ and the boundedness of $f$ that

$$
\left|\alpha_{n}\right| \leq C
$$

Let us check that

$$
\int F_{n}=\int F_{n-1}
$$

also when $Q_{n} \in$ PLI. Replace $\rho_{n-1}$ by its value $\rho_{n}^{*}$ on $Q_{n}$ in (4.36), and also recall that $\left\|\nu_{n}\right\|=\rho_{n}^{*} \mu\left(H_{n}\right)$ by (4.37); we see that (4.39) is the same as

$$
\alpha_{n} \rho_{n}^{*} \mu\left(H_{n}\right)=\rho_{n}^{*} \int_{H_{n}} f d \mu+\rho_{n}^{*} \theta_{n} \int_{G_{n}} f d \mu
$$

Now (4.40) holds because of (4.34) and (4.28) for the real part, and (4.35) for the imaginary part.

Now we wish to define our function $g$ and measure $\nu$. Of course $g d \nu$ will be the limit of the measures $F_{n}$, but it will be better to spend some time and define $g$ and $\nu$ quietly.

First observe that the functions $\rho_{n}$ are a decreasing sequence of nonnegative functions on $E$. Denote by $\rho_{\infty}$ the limit of this sequence. Obviously, $0 \leq \rho_{\infty} \leq 1$ on $E$. Also set $d \mu_{\infty}=\rho_{\infty} d \mu$. This is the first half of $d \nu$. The second half is the sum of the measures $d \nu_{n}$. In other words, we take

$$
d \nu=d \mu_{\infty}+\sum_{n} d \nu_{n}=\rho_{\infty} d \mu+\sum_{n} d \nu_{n}
$$

Set

$$
E_{\infty}=\left\{x \in E: \rho_{\infty}(x) \neq 0\right\}
$$

Our intention is to take $g(x)=f(x)$ on $E_{\infty}$ and $g(x)=\alpha_{n}$ on $\mathcal{C}_{n}$, but this will be more pleasant to do once we know that those sets are disjoint. It will also be useful to know that they are reasonably far from each other.

Lemma 4.43. We have that

$$
\operatorname{dist}\left(\mathcal{C}_{n}, E_{\infty}\right) \geq \frac{1}{2} A^{-k_{n}}
$$


and

$$
\operatorname{dist}\left(\mathcal{C}_{n}, \mathcal{C}_{m}\right) \geq \frac{1}{4} \max \left\{A^{-k_{n}}, A^{-k_{m}}\right\}
$$

for all $n$ and $m \neq n$.

First suppose that $Q_{n} \in I_{1}$. Then $\mathcal{C}_{n}=\partial\left(B\left(Q_{n}\right) / 2\right)$ and $k_{n}=$ $k\left(Q_{n}\right)$. In this case,

$$
\operatorname{dist}\left(\mathcal{C}_{n}, E \backslash Q_{n}\right) \geq \frac{1}{2} A^{-k_{n}}
$$

by (4.3), and (4.44) follows from this (recall that $\rho(x) \equiv 0$ on $Q_{n}$ by (4.18)).

When $Q_{n} \in$ PLI, $\mathcal{C}_{n}=\mathcal{C}\left(Q_{n}^{*}\right)$ for some child $Q_{n}^{*}$ of $Q_{n}$, and $k_{n}=$ $k\left(Q_{n}^{*}\right)$ by (4.14). Since $H_{n}$ contains $Q_{n}^{*}$ because $Q_{n}^{*} \in$ LI, (4.3) implies that

$$
\operatorname{dist}\left(\mathcal{C}_{n}, E \backslash H_{n}\right) \geq \frac{1}{2} A^{-k_{n}},
$$

(4.44) follows from this because $\rho(x)=0$ on $H_{n}$, by (4.27). Thus (4.44) holds in all cases.

Clearly it is enough to prove (4.45) when $n<m$. By definition of the order on $I, k_{m} \geq k_{n}$ and the maximum in (4.45) is $A^{-k_{n}}$. Let $Q$ and $Q^{\prime}$ be the cubes such that $\mathcal{C}_{n}=\mathcal{C}(Q)$ and $\mathcal{C}_{m}=\mathcal{C}\left(Q^{\prime}\right)$. Thus $Q$ is $Q_{n}$ itself, or a child $Q_{n}^{*}$ if $Q_{n} \in$ PLI, and similarly for $Q^{\prime}$. A first case is when $k_{m}=k_{n}$, i.e., $Q$ and $Q^{\prime}$ are of the same generation $k_{n}$. Then $Q$ and $Q^{\prime}$ are disjoint (the only "dangerous" case would be when $Q_{n} \in$ PLI and $Q^{\prime} \subset Q_{n}$, but then $Q^{\prime} \in I_{1}$ and $Q \in$ LI so they are disjoint). In this case we may use the fact that $5 B(Q)$ and $5 B\left(Q^{\prime}\right)$ are disjoint (by (3.9)), and (4.45) follows from the lower bound (3.7) on the radii of $B(Q)$ and $B\left(Q^{\prime}\right)$.

When $k_{m}>k_{n}, Q^{\prime}$ is still disjoint from $Q$ (because they are both cubes of $I_{1} \cup \mathrm{LI}$, and they are different because they are from different generations). Then dist $\left(Q^{\prime}, \mathcal{C}_{n}\right) \geq A^{-k_{n}} / 2$ by (4.3), and since the center of $\mathcal{C}_{m}$ belongs to $Q^{\prime}$ and $\operatorname{diam} \mathcal{C}_{m} \leq C_{0} A^{-k_{m}}<A^{-k_{n}} / 4$ by (3.7), we get (4.45). This completes the proof of Lemma 4.43.

An easy consequence of this lemma is that the $\operatorname{circles} \mathcal{C}_{n}$ are disjoint from each other and from $E_{\infty}$. This allows us to define $g$ on $E_{\infty} \cup$ $\left(\bigcup_{n} \mathcal{C}_{n}\right)$ by

$$
\begin{cases}g(x)=\alpha_{n}, & \text { on } \mathcal{C}_{n}, \\ g(x)=f(x), & \text { on } E_{\infty} .\end{cases}
$$


Next we want to check that $g$ and $\nu$ satisfy the properties required for Theorem 2.4, except for the last one which is related to the Cauchy kernel.

\subsection{The easy properties of $g d \nu$.}

We shall start with the upper-regularity condition (2.5). From now on throughout the rest of the paper $C$ will denote constants which may change from line to line and which may depend only on $C_{0}, A, M, a$, $a_{0}, a_{1}, \mu(E)$ and $M_{0}$, defined in (4.87). (But recall that we can fix $C_{0}$ and $A$ as large absolute constants and $a_{0}$ and $a_{1}$ will depend only on $a^{-1} \mu(E)$.)

Lemma 4.49. There is a constant $C>0$ such that

$$
\mu_{\infty}(B(x, r)) \leq C r, \quad \text { for all } x \in \mathbb{C} \text { and } r>0 .
$$

Since $E$ is compact (and even has diameter at most 1 by our earlier normalization), it is enough to prove (4.50) when $r \leq 1$. Cover $E \cap$ $B(x, r)$ by cubes $Q \in \Delta_{k}$, where $k$ is chosen so that $A^{-\bar{k}} \sim r$. You need less than $C$ cubes $Q$, because all the balls $5 B(Q)$ are disjoint and have radii greater or equal to $5 A^{-k}$, while the balls $28 B(Q)$ all meet $B(x, r)$ (when $Q$ meets it) and have radii at most $28 C_{0} A^{-k}$. We have that $\mu(Q) \leq C A^{-k} \leq C r$ for all the cubes of our collection that are not contained in any cube of $I_{1} \cup \mathrm{LI}$, by Remark 4.12. (If $\mu(Q)>C A^{-k}$ with $C$ large enough, then $Q$ is contained in $\mathcal{O}(M)$.) So the total mass of these cubes is at most $C r$. On the other hand, if $Q$ is contained in some cube of $I_{1} \cup \mathrm{LI}$, then $\rho_{\infty} \equiv 0$ on $Q$ (because $\rho_{\infty}(x)=0$ on all cubes of $I_{1} \cup \mathrm{LI}$, by (4.18) and (4.27)). Hence the total mass of these cubes for $\mu_{\infty}$ is zero. This proves the lemma.

Now we want to control the measures $\nu_{n}$. We start with estimates on the position of the cubes $Q_{n}$. We claim that

$$
\sum_{\substack{Q \in I_{1} \cup \mathrm{LI} \\ Q \operatorname{meets} B(x, r) \\ A^{-k(Q)} \leq r}} \mu(Q) \leq C r,
$$

for all $x \in \mathbb{C}$ and $r>0$. 
Since $\operatorname{diam} E \leq 1$ by our earlier normalization, we can safely suppose that $r \leq 1$. If $Q \in I_{1} \cup \mathrm{LI}$, then its parent $\widehat{Q}$ is not contained in $\mathcal{O}(M)$; this follows from Remark 4.12 and the disjointness property (4.11). If $Q$ meets $B(x, r)$ and $A^{-k(Q)} \leq r$, then $\widehat{Q} \subset B(x, C r)$ if $C$ is large enough, and then $B(x, C r)$ is not contained in $\mathcal{O}(M)$ either. Thus either the sum in (4.51) is empty, or else $\mu(B(x, r)) \leq \mu(B(x, C r)) \leq$ $M C r$, and (4.51) follows from the disjointness property (4.11).

Let us also record that

$$
\sum_{\substack{Q_{n} \in \mathrm{PLI} \\ Q_{n} \subset B(x, r)}} \mu\left(H_{n}\right) \leq C r
$$

for all $x \in \mathbb{C}$ and $r>0$. This is an easy consequence of the proof of (4.51), because the cubes $Q_{n}$ are not contained in $\mathcal{O}(M)$ and the sets $H_{n}$ are obtained from the cubes of LI by regrouping brothers.

We are now ready to prove that $\nu$ satisfies (2.5). Recall from (4.41) that $\nu=\mu_{\infty}+\sum_{n} \nu_{n}$. Because of Lemma 4.49, it is enough to show that

$$
\sum_{n} \nu_{n}(B(x, r)) \leq C r, \quad \text { for all } x \in \mathbb{C} \text { and } r>0
$$

Since by construction all the $\operatorname{circles} \mathcal{C}_{n}$ lie in a fixed ball of radius $C$ (and even with $C=1$ ), it is enough to prove (4.53) when $r \leq 1$. Let $B(x, r)$ be given, and start with the set $\mathcal{N}_{1}$ of integers $n$ for which $Q_{n} \in I_{1}, \mathcal{C}_{n}$ meets $B(x, r)$, and $A^{-k\left(Q_{n}\right)} \leq r$. Then

$$
\sum_{n \in \mathcal{N}_{1}} \nu_{n}(B(x, r)) \leq \sum_{n \in \mathcal{N}_{1}}\left\|\nu_{n}\right\| \leq \sum_{n \in \mathcal{N}_{1}} \mu\left(Q_{n}\right) \leq C r
$$

by (4.21) and (4.51). Similarly, if $\mathcal{N}_{2}$ is the set of integers $n$ for which $Q_{n} \in$ PLI and $Q_{n} \subset B(x, 2 r)$, then

$$
\sum_{n \in \mathcal{N}_{2}} \nu_{n}(B(x, r)) \leq \sum_{n \in \mathcal{N}_{2}}\left\|\nu_{n}\right\| \leq \sum_{n \in \mathcal{N}_{2}} \mu\left(H_{n}\right) \leq C r
$$

by (4.37) and (4.52).

Thus we are left with the set $\mathcal{N}_{3}$ of integers $n$ for which $\mathcal{C}_{n}$ meets $B(x, r)$ and either $Q_{n} \in I_{1}$ and $A^{-k\left(Q_{n}\right)}>r$ or $Q_{n} \in$ PLI and is not contained in $B(x, 2 r)$. In this last case we also have that $A^{-k_{n}} \geq r / C$, and (4.45) tells us that $\mathcal{N}_{3}$ has at most $C$ elements. Thus it is enough to 
prove that $\nu_{n}(B(x, r)) \leq C r$ for each single $\mathcal{C}_{n}$. This is an immediate consequence of the fact that the densities

$$
\rho_{n}^{*} \frac{\mu\left(Q_{n}\right)}{H^{1}\left(\mathcal{C}_{n}\right)} \quad \text { and } \quad \rho_{n}^{*} \frac{\mu\left(H_{n}\right)}{H^{1}\left(\mathcal{C}_{n}\right)}
$$

in (4.17) and (4.26) are less than $C$, which follows from (4.13) applied to $Q_{n}$.

Thus $\nu$ satisfies the upper regularity condition (2.5).

The constant that we obtain in (2.5) may depend wildly on $E$, because it depends on our initial choice of $M$, as in (4.6). On the other hand, if we start from a measure $\mu$ that already satisfies (2.5) with a constant $C_{1}$, then we can take $M \leq C C_{1}$ and $\nu$ satisfies (2.5) with a constant at most $C^{\prime} C_{1}$, where $C$ and $C^{\prime}$ are absolute constants. This follows from the proof above, together with the fact that the constants that come from the construction of dyadic cubes can be taken to be absolute constants.

Next we want to prove the accretivity condition (2.6). When $x \in$ $\mathcal{C}_{n}, g(x)=\alpha_{n}$, and so $\left|\alpha_{n}\right| \leq C$ (by (4.23) or (4.38)) and $\operatorname{Re} \alpha_{n} \geq a_{0}$ (by (4.24) or (4.34)). When $x \in E_{\infty}, g(x)=f(x)$ and so $|g(x)| \leq 1$ by (2.2). We shall not be able to prove that $\operatorname{Re} f(x) \geq a_{0}$ everywhere on $E_{\infty}$, but only $\mu$-almost everywhere. Of course this is enough, because modifying $g$ on a subset of $\mu$-measure zero of $E_{\infty}$ will not alter the other properties required for the theorem. We shall use a simple variant of the Lebesgue density theorem.

Lemma 4.56. For each $x \in E$ and each $k \geq 0$, denote by $Q(x, k)$ the cube of $\Delta_{k}$ that contains $x$. Then

$$
f(x)=\lim _{k \rightarrow+\infty}\left(\mu(Q(x, k))^{-1} \int_{Q(x, k)} f d \mu\right),
$$

for $\mu$-almost every $x \in E$.

Let us first say why (2.6) follows from this lemma. Let $x \in E$ be such that (4.57) holds. If $\operatorname{Re} f(x)<a_{0}$, we can find a small cube $Q$ that contains $x$ and for which $\operatorname{Re} \int_{Q} f d \mu<a_{0} \mu(Q)$. Then Remark 4.12 says that $Q$ is contained in a cube of $I_{1} \cup$ LI. Since $\rho(x)=0$ or all cubes of $I_{1} \cup$ LI (by the definition of $\rho_{n}$ on $Q_{n}$ ), $x$ cannot lie in $E_{\infty}$. This proves that $\operatorname{Re} f(x) \geq a_{0}$ (and hence $\left.\operatorname{Re} g(x) \geq a_{0}\right) \mu$-almost everywhere on $E_{\infty}$. This completes the proof of (2.6), modulo the lemma. 
The proof of Lemma 4.56 is standard. We certainly have (4.57) everywhere for all continuous functions $f$. Because of the standard approximation argument, it is enough to prove a weak- $L^{1}$ estimate for the associated maximal function. Let us make this more precise.

For each $L^{1}$-function $h$ on $E$, define the maximal function $M h$ by

$$
M h(x)=\sup _{k \geq 0}\left(\mu(Q(x, k))^{-1} \int_{Q(x, k)}|h| d \mu\right),
$$

for all $x \in E$, and where $Q(x, k)$ is as in the statement of Lemma 4.56.

For each $\lambda>0$, set $U(\lambda)=\{x \in E: M h(x)>\lambda\}$, and denote by $\Delta(\lambda)$ the set of maximal cubes of $\Delta=\bigcup_{k} \Delta_{k}$ such $\int_{Q}|h| d \mu>\lambda \mu(Q)$. Each cube of $\Delta(\lambda)$ is clearly contained in $U(\lambda)$, and conversely every point of $U(\lambda)$ lies in a $Q \in \Delta(\lambda)$. Thus $U(\lambda)$ is the disjoint union of the cubes of $\Delta(\lambda)$, and

$$
\begin{aligned}
\mu(U(\lambda)) & =\sum_{Q \in \Delta(\lambda)} \mu(Q) \\
& \leq \lambda^{-1} \sum_{Q \in \Delta(\lambda)} \int_{Q}|h| d \mu \\
& \leq \lambda^{-1} \int_{E}|h| d \mu .
\end{aligned}
$$

This is the weak- $L^{1}$ estimate that we needed to deduce (4.57) for all $f \in L^{1}$ from the case of continuous functions. This completes our proof of (2.6).

The next property (2.7) follows from (2.2), (4.22) and (4.39) by taking limits. The reader should not worry about convergence: it is easy to see that $\sum_{n}\left\|F_{n+1}-F_{n}\right\|$ converges by (4.15), the fact that $\mu$ is finite and the sequence $\left\{\rho_{n}\right\}$ is decreasing, and because $\left|\alpha_{n}\right| \leq C$ and

$$
\sum\left\|\nu_{n}\right\| \leq \sum_{Q \in I_{1}} \mu(Q)+\sum_{Q_{n} \in \mathrm{PLI}} \mu\left(H_{n}\right)<+\infty
$$

by (4.21), (4.37), (4.51) and (4.52) (or directly (4.11).)

Next we want to prove (2.8). Set

$$
F=\left\{x \in E_{\infty}: \rho_{\infty}(x) \geq \tau\right\}
$$


where $\tau=a /(4 \mu(E))$. Note that $d \nu=\rho_{\infty} d \mu$ on $F$ because $F \subset E_{\infty}$ and the circles $\mathcal{C}_{n}$ live away from $E_{\infty}$. Clearly $\tau d \mu \leq d \nu \leq d \mu$ on $F$, and so it is enough to prove that $\nu(F) \geq a / 2$.

We know from (2.7) that $\int g d \nu=a$. Also $\nu(F) \geq \int_{F} \operatorname{Re} f d \nu=$ $\int_{F} \operatorname{Re} g d \nu$ because $|f| \leq 1$ and $f=g$ on $E_{\infty}$. Thus it is enough to prove that

$$
\operatorname{Re} \int g d \nu-\operatorname{Re} \int_{F} g d \nu \leq \frac{a}{2}
$$

Recall that $d \nu=\rho_{\infty} d \mu+\sum_{n} d \nu_{n}$ and that $g(x)=f(x)$ on $E_{\infty}$ and $g(x)=\alpha_{n}$ on $\mathcal{C}_{n}$. Then (4.61) is equivalent to

$$
\operatorname{Re} \int_{E_{\infty} \backslash F} f \rho_{\infty} d \mu+\sum_{n}\left\|\nu_{n}\right\| \operatorname{Re} \alpha_{n} \leq \frac{a}{2} .
$$

Note that $\rho_{\infty}(x) \leq \tau$ on $E_{\infty} \backslash F$, by the definition of $F$. Hence

$$
\operatorname{Re} \int_{E_{\infty} \backslash F} f \rho_{\infty} d \mu \leq\|f\|_{\infty} \tau \mu(E) \leq \frac{a}{4}
$$

When $Q_{n} \in$ PLI, $\operatorname{Re} \alpha_{n}=a_{0}$ by (4.34) and $\left\|\nu_{n}\right\| \leq \mu\left(H_{n}\right)$ by (4.37). Then

$$
\left\|\nu_{n}\right\| \operatorname{Re} \alpha_{n} \leq a_{0} \mu\left(H_{n}\right), \quad \text { when } Q_{n} \in \mathrm{PLI} \text {. }
$$

When $Q_{n} \in I_{1},\left\|\nu_{n}\right\|=\rho_{n}^{*} \mu\left(Q_{n}\right)$ by (4.21), where $\rho_{n}^{*}$ is the constant value of $\rho_{n-1}$ on $Q_{n}$, and $\alpha_{n}=\mu\left(Q_{n}\right)^{-1} \int_{Q_{n}} f d \mu$ by (4.19). So

$$
\left\|\nu_{n}\right\| \operatorname{Re} \alpha_{n}=\rho_{n}^{*} \operatorname{Re} \int_{Q_{n}} f d \mu, \quad \text { when } Q_{n} \in I_{1} .
$$

If furthermore $Q_{n} \in \mathrm{MI}$, then it satisfies (4.7) and so $\left\|\nu_{n}\right\| \operatorname{Re} \alpha_{n} \leq$ $\rho_{n}^{*} a_{1} \mu\left(Q_{n}\right) \leq a_{1} \mu\left(Q_{n}\right)$. Otherwise we can only say that $\left\|\nu_{n}\right\| \operatorname{Re} \alpha_{n} \leq$ $\mu\left(Q_{n}\right)$ (because $\left.|f| \leq 1\right)$, but we have the advantage of knowing that $Q \in$ HD. Altogether,

$$
\begin{aligned}
\sum_{n}\left\|\nu_{n}\right\| \operatorname{Re} \alpha_{n} \leq & a_{0} \sum_{Q_{n} \in \mathrm{PLI}} \mu\left(H_{n}\right)+a_{1} \sum_{Q_{n} \in I_{1} \cap \mathrm{MI}} \mu\left(Q_{n}\right) \\
& +\sum_{Q_{n} \in I_{1} \cap \mathrm{HD}} \mu\left(Q_{n}\right) .
\end{aligned}
$$


Recall from (4.11) that the cubes of $I_{1} \cup \mathrm{LI}$ are disjoint. Also,

$$
\sum_{Q_{n} \in \mathrm{PLI}} \mu\left(H_{n}\right)=\sum_{Q \in \mathrm{LI}} \mu(Q)
$$

because the sets $H_{n}$ are obtained from the set of cubes of LI by regrouping brothers. Hence

$$
a_{0} \sum_{Q_{n} \in \mathrm{PLI}} \mu\left(H_{n}\right)+a_{1} \sum_{Q_{n} \in I_{1}} \mu\left(Q_{n}\right) \leq a_{1} \mu(E)
$$

and we can make this at most $a / 8$ by taking $a_{1}$ small enough. We can choose $a_{1}=a /(8 \mu(E))$, for instance.

The last sum is taken care of by noticing that the cubes of $I_{1} \cap \mathrm{HD}$ are disjoint and contained in $\mathcal{O}(M)$. Then

$$
\sum_{Q_{n} \in I_{1} \cap \mathrm{HD}} \mu\left(Q_{n}\right) \leq \mu(\mathcal{O}(M))<\frac{a}{8}
$$

by (4.6).

The desired estimate (4.62) follows from (4.67), (4.68), our choice of $a_{1},(4.66)$ and (4.63). This completes our proof of (2.8).

We did not choose $a_{0}$ yet. In fact, any value of $a_{0}$ in $\left(0, a_{1}\right)$ will work, but for the sake of definiteness let us choose $a_{0}=a_{1} / 2$.

We are now finished with the verification of the "easy properties" of $g$ and $\nu$. Note that the constant $C$ in (2.6) and (2.8) can be taken to be $\leq C_{1} a^{-1} \mu(E)$ for some absolute constant $C_{1}$, as we announced after the statement of Theorem 2.4. The remaining property, i.e., the $L^{2}$-estimate for the maximal Cauchy integral of $g d \nu$, will keep us busy for the rest of the paper.

\section{3. $L^{2}$-estimates: reduction to positive functions.}

Our proof that $T^{*}(g d \nu) \in L^{2}(d \nu)$ will be in the same spirit as the estimates in [C2], even if we pay a rather high price for the fact that we are not working with a space of homogeneous type.

In this section we reduce the proof of (2.9) to the study of certain positive functions. The $L^{2}$-estimates for these functions will be derived in the last chapter, using the small boundary properties of our cubes (which we did not use so far). 
We start with a few notations. First, it will be convenient to define $H_{n}$ even when $Q_{n} \in I_{1}$. In this case we simply set $H_{n}=Q_{n}$. Also set $G_{n}=\varnothing$ when $Q_{n} \in I_{1}$. With this convention,

the sets $H_{n}, n \in I$, are disjoint,

because the sets $H_{n}, Q_{n} \in \mathrm{PLI}$, were obtained from the cubes $Q \in \mathrm{LI}$ by regrouping brothers, and by (4.11). Also

$$
\sum_{\substack{Q_{n} \subset B(x, r) \\ A^{-k_{n}} \leq r}} \mu\left(H_{n}\right) \leq C r
$$

for all $x \in \mathbb{C}$ and $r>0$, by (4.51) and (4.52). Set

$$
\varphi_{n}=F_{n}-F_{n-1}
$$

for $n \geq 1$. With our unified notations, (4.20) and (4.36) reduce to

$$
\varphi_{n}=-\mathbf{1}_{H_{n}} \rho_{n-1} f d \mu-\theta_{n} \mathbf{1}_{G_{n}} \rho_{n-1} f d \mu+\alpha_{n} d \nu_{n} .
$$

We do not have to define $\theta_{n}$ when $Q_{n} \in I_{1}$, since $G_{n}$ is then empty. We shall often use the estimates

$$
\left|\alpha_{n}\right| \leq C \quad \text { and } \quad\left\|\nu_{n}\right\| \leq \mu\left(H_{n}\right),
$$

which follow from (4.21), (4.23), (4.37) and (4.38).

Denote by BLI (brothers of LI) the union of all the sets $\mathcal{A}_{n}^{*}$, where $Q_{n} \in$ PLI. (The definition of $\mathcal{A}_{n}^{*}$ is given just before (4.25).) This is the set of the children of the cubes of PLI that are not in LI. We should be a little more careful with the cubes of BLI, because they are not necessarily disjoint. Set

$$
\begin{cases}\theta(Q)=1, & \text { when } Q \in I_{1} \cup \mathrm{LI}, \\ \theta(Q)=\theta_{n}, & \text { when } Q \in \mathcal{A}_{n}^{*} \text { and } Q_{n} \in \mathrm{PLI} .\end{cases}
$$

There is a potential conflict here, because a given cube $Q$ could be in BLI and in $I_{1}$ at the same time. In this case $\theta(Q)$ will be defined twice. This will not be a problem; it will always be clear from the context which $\theta(Q)$ we need to take, and the worse thing that may happen is that a given cube will appear twice in a same sum, once with $\theta(Q)=1$ and once with $\theta(Q)=\theta_{n}$. 
Note that for each $Q_{n} \in$ PLI,

$$
\sum_{Q \in \mathcal{A}_{n}^{*}} \theta(Q) \mu(Q)=\theta_{n} \mu\left(G_{n}\right) \leq C \mu\left(H_{n}\right)
$$

by (4.33). Then

$$
\sum_{\substack{Q \in \mathrm{BLI} \\ Q \subset B(x, r) \\ A^{-k(Q)} \leq r}} \theta(Q) \mu(Q) \leq C r,
$$

when $x \in \mathbb{C}$ and $r>0$, because the left-hand side of (4.76) is less than

$$
\sum_{\substack{Q_{n} \in \mathrm{PLI} \\ Q_{n} \subset B(x, C r)}} \sum_{Q \in \mathcal{A}_{n}^{*}} \theta(Q) \mu(Q),
$$

which is less than $C r$ by (4.75) and (4.52).

We are now ready to define the positive functions that will control $T^{*}(g d \nu)$. For each cube $Q \in \Delta$, define a normalized inverse distance function $\rho_{Q}$ by

$$
\rho_{Q}(x)=\left(1+A^{k(Q)} \operatorname{dist}(x, Q)\right)^{-1},
$$

and then set

$$
e_{Q}^{*}(x)=\rho_{Q}(x) \int_{Q} \frac{d \mu(y)}{|x-y|},
$$

for all $x \in \mathbb{C}$. Also set

$$
2 Q=\left\{y \in E: \operatorname{dist}(y, Q) \leq A^{-k(Q)}\right\}
$$

and

$$
h_{Q}^{*}(x)=\int_{2 Q \backslash Q} \frac{d \mu(y)}{|x-y|} .
$$

The next proposition gives the control on $T^{*}(g d \nu)$ that we want on $E_{\infty}$. 
Proposition 4.81. If $x \in E_{\infty}$, then

$$
\begin{aligned}
T^{*}(g d \nu)(x) \leq & C+C \sum_{Q \in I_{1} \cup \mathrm{LI} \cup \mathrm{BLI}} \theta(Q) \mathbf{1}_{E \backslash Q}(x) e_{Q}^{*}(x) \\
& +C \sum_{Q \in \mathrm{BLI}} \theta(Q) \mathbf{1}_{Q}(x) h_{Q}^{*}(x) .
\end{aligned}
$$

We shall also have to estimate $T^{*}(g d \nu)$ on the circles $\mathcal{C}_{n}$, and so we need a variant of Proposition 4.81 for $x \in \mathcal{C}_{n}$. For each $Q \in I_{1} \cup L I \cup B L I$, let $J(Q)$ denote the set of integers $m \geq 1$ such that $Q_{m}$ does not meet $Q$, or else $Q_{m} \in$ PLI and $Q \subset G_{m}$. Then set

$$
\widetilde{e}_{Q}(x)=\sum_{n \in J(Q)} \mathbf{1}_{\mathcal{C}_{n}}(x) e_{Q}^{*}(x),
$$

and also

$$
\widetilde{h}_{Q}(x)=\sum_{n: Q_{n} \subset Q} \mathbf{1}_{\mathcal{C}_{n}}(x) h_{Q}^{*}(x),
$$

when $Q \in$ BLI.

Proposition 4.85. If $x \in \bigcup_{n} \mathcal{C}_{n}$, then

$$
\begin{aligned}
T^{*}(g d \nu)(x) \leq & C+C \sum_{Q \in I_{1} \cup \mathrm{LI} \cup \mathrm{BLI}} \theta(Q) \widetilde{e}_{Q}(x) \\
& +C \sum_{Q \in \mathrm{BLI}} \theta(Q) \widetilde{h}_{Q}(x) .
\end{aligned}
$$

In this section we shall prove the two propositions; the functions $e_{Q}^{*}$ and $h_{Q}^{*}$ will be estimated in Part 5 .

First we want to use the boundedness of $T(f d \mu)$ to get estimates on truncated integrals. Our assumption from Chapter 2 says that

$$
M_{0}=\sup \{|T(f d \mu)(x)|: x \in \mathbb{C} \backslash E\}<+\infty,
$$

where $T(f d \mu)$ is as in (2.3).

For each finite complex measure $\varphi$ on $\mathbb{C}$ and all $\varepsilon>0$, set

$$
T^{\varepsilon} \varphi(x)=\int_{\mathbb{C} \backslash(x, \varepsilon)} \frac{d \varphi(y)}{x-y},
$$


for all $x \in \mathbb{C}$.

Lemma 4.89. We have that

$$
\left|T^{\varepsilon}(f d \mu)(x)\right| \leq M_{0}+C \sup _{r>\varepsilon}\left(r^{-1} \mu(B(x, r))\right),
$$

for all $x \in \mathbb{C}$.

To prove this, let $x$ and $\varepsilon$ be given, and set

$$
h(z)=\int_{\mathbb{C} \backslash B(x, \varepsilon)} \frac{f(y) d \mu(y)}{z-y},
$$

for $z \in B(x, \varepsilon / 2)$. Then

$$
\begin{aligned}
\left|T^{\varepsilon}(f d \mu)(x)-h(z)\right| & \leq \int_{\mathbb{C} B(x, \varepsilon)} \frac{|z-x| d \mu(y)}{|z-y||x-y|} \\
& \leq 4 \sup _{r>\varepsilon}\left(r^{-1} \mu(B(x, r))\right)
\end{aligned}
$$

by the usual computation. (Cut the domain of integration into annuli $A_{k}:\left\{y: 2^{k} \varepsilon \leq|x-y|<2^{k+1} \varepsilon\right\}, k=0,1, \ldots$, note that

$$
\frac{|z-x|}{|z-y||x-y|} \leq 2^{-2 k} \varepsilon^{-1}
$$

on $A_{k}$ and $\mu\left(A_{k}\right) \leq 2^{k+1} \varepsilon \sup _{r>\varepsilon}\left(r^{-1} \mu(B(x, r))\right)$, and sum a geometric series.) Also, if $z \in B(x, \varepsilon / 2) \backslash E$,

$$
|T(f d \mu)(z)-h(z)|=\left|\int_{B(x, \varepsilon)} \frac{f(y) d \mu(y)}{z-y}\right| \leq \int_{B(x, \varepsilon)} \frac{d \mu(y)}{|z-y|} .
$$

The mean value on $B(x, \varepsilon / 2) \backslash E$ of this last integral is (with $|B|$ denoting the Lebesgue measure of $B$ )

$$
\begin{aligned}
\left|B\left(x, \frac{\varepsilon}{2}\right)\right|^{-1} \int_{B(x, \varepsilon / 2) \backslash E}\left(\int_{B(x, \varepsilon)} \frac{d \mu(y)}{|z-y|}\right) d z \\
\leq 4 \varepsilon^{-2} \int_{B(x, \varepsilon)}\left(\int_{B(x, \varepsilon / 2)} \frac{d z}{|z-y|}\right) d \mu(y) \\
\leq C \varepsilon^{-1} \mu(B(x, \varepsilon)) .
\end{aligned}
$$


Thus we may choose $z \in B(x, \varepsilon / 2) \backslash E$ so that $|T(f d \mu)(z)-h(z)| \leq$ $C \varepsilon^{-1} \mu(B(x, \varepsilon))$. Since we already know that $T(f d \mu)(z) \leq M_{0},(4.90)$ follows from this and (4.91).

Recall from (4.18) and (4.27) that $\rho(x)=0$ on all cubes of $I_{1} \cup \mathrm{LI}$. Thus

$$
E_{\infty} \text { does not meet any cube of } I_{1} \cup \mathrm{LI},
$$

and Remark 4.12 says that $E_{\infty}$ does not even meet any cube $Q$ such that $Q \subset \mathcal{O}(M)$. (See the definitions (4.4) and (4.5).) From this we deduce that

$$
\mathcal{M}_{\mu}(x)=\sup _{r>0}\left(r^{-1} \mu(B(x, r))\right) \leq C, \quad \text { for } x \in E_{\infty} .
$$

Indeed, we can take $C=2 M$ because if $\mu(B(x, r))>2 M r$, then $\mu(B(y, 2 r))>M 2 r$ for $y \in B(x, r)$, whence $B(x, r) \subset \mathcal{O}(M)$ and all sufficiently small cubes containing $x$ would also be contained in $\mathcal{O}(M)$.

When $x \in \mathcal{C}_{n}$ for some $n$, we may use the fact that the parent $\widehat{Q}_{n}$ of $Q_{n}$ is not contained in $I_{1} \cup \mathrm{LI}$, plus Remark 4.12 , to find that $\widehat{Q}_{n}$ is not contained in $\mathcal{O}(M)$. Because of this

$$
\mu(B(x, r)) \leq C r, \quad \text { when } x \in \mathcal{C}_{n} \text { and } r \geq A^{-k_{n}} .
$$

(See near (4.14) for the definition of $k_{n}$.)

From (4.95), (4.96) and Lemma 4.89 we deduce that

$$
T^{*}(f d \mu)(x) \leq C, \quad \text { for } x \in E_{\infty}
$$

and

$$
\left|T^{\varepsilon}(f d \mu)(x)\right| \leq C, \quad \text { for } x \in \mathcal{C}_{n} \text { and } \varepsilon \geq \frac{A^{-k_{n}}}{5}
$$

say.

Now we want to estimate $T^{\varepsilon} \varphi_{n}$, with $\varphi_{n}=F_{n}-F_{n-1}$, when $x \in E_{\infty}$ or $x \in \mathcal{C}_{m}$ for some $m \neq n$. In both cases Lemma 4.43 says that dist $\left(x, \mathcal{C}_{n}\right) \geq A^{-k_{n}} / 4$. Let us first use (4.72) and estimate brutally

$$
\begin{aligned}
\left|T^{\varepsilon} \varphi_{n}(x)\right| & \leq \int_{H_{n}} \frac{d \mu(y)}{|x-y|}+\theta_{n} \int_{G_{n}} \frac{d \mu(y)}{|x-y|}+\frac{C \mu\left(H_{n}\right)}{\operatorname{dist}\left(x, \mathcal{C}_{n}\right)} \\
& \leq C \int_{H_{n}} \frac{d \mu(y)}{|x-y|}+\theta_{n} \int_{G_{n}} \frac{d \mu(y)}{|x-y|}
\end{aligned}
$$


by (4.73), and then the fact that $1 /|x-y| \geq C^{-1} \operatorname{dist}\left(x, \mathcal{C}_{n}\right)^{-1}$ for all $y \in H_{n}$, because dist $\left(x, \mathcal{C}_{n}\right) \geq A^{-k_{n}} / 4$ and $\operatorname{diam}\left(H_{n} \cup \mathcal{C}_{n}\right) \leq C A^{-k_{n}}$.

We do not want to use (4.99) in all cases. In particular, when $x$ is far from $Q_{n}$, we wish to "integrate by parts" and get an estimate with a better decay. Let us assume that dist $\left(x, Q_{n}\right) \geq A^{-k_{n}+2}$, say, and that $\varepsilon \leq \operatorname{dist}\left(x, Q_{n} \cup \mathcal{C}_{n}\right)$. In this case

$$
T^{\varepsilon} \varphi_{n}(x)=\int \frac{d \varphi_{n}(y)}{x-y}
$$

i.e., there is no truncation. Pick any point $y_{0} \in \mathcal{C}_{n}$; then

$$
\int \frac{d \varphi_{n}(y)}{x-y_{0}}=0
$$

because $\int d \varphi_{n}=0$, by (4.22) or (4.39). Hence

$$
\begin{aligned}
\left|T^{\varepsilon} \varphi_{n}(x)\right| & =\left|\int \frac{\left(y-y_{0}\right) d \varphi_{n}(y)}{(x-y)\left(x-y_{0}\right)}\right| \\
& \leq\left\|\varphi_{n}\right\| \operatorname{diam}\left(Q_{n} \cup \mathcal{C}_{n}\right) \operatorname{dist}\left(x, Q_{n} \cup \mathcal{C}_{n}\right)^{-2} \\
& \leq C \mu\left(H_{n}\right) A^{-k_{n}} \operatorname{dist}\left(x, Q_{n}\right)^{-2}
\end{aligned}
$$

by (4.72), (4.73), and the end of (4.75) when $Q_{n} \in$ PLI.

When $\operatorname{dist}\left(x, Q_{n}\right) \geq A^{-k_{n}+2}$ but

$$
\operatorname{dist}\left(x, Q_{n} \cup \mathcal{C}_{n}\right)<\varepsilon<\operatorname{dist}\left(x, Q_{n} \cup \mathcal{C}_{n}\right)+\operatorname{diam}\left(Q_{n} \cup \mathcal{C}_{n}\right)
$$

we may have a problem of truncation. In this case we shall prefer to use (4.99) which takes the simpler form

$$
\left|T^{\varepsilon} \varphi_{n}(x)\right| \leq C \mu\left(H_{n}\right) \operatorname{dist}\left(x, Q_{n}\right)^{-1}
$$

(we still use (4.75) here when $Q_{n} \in \mathrm{PLI}$ ). Set

$$
\begin{aligned}
T(n, \varepsilon)=\{x \in \mathbb{C}: & \operatorname{dist}\left(x, Q_{n}\right) \geq A^{-k_{n}+2} \\
& \text { and }(4.101) \text { holds }\}
\end{aligned}
$$

Our last case is when $\varepsilon \geq \operatorname{dist}\left(x, Q_{n} \cup \mathcal{C}_{n}\right)+\operatorname{diam}\left(Q_{n} \cup \mathcal{C}_{n}\right)$. In this case $T^{\varepsilon} \varphi_{n}(x)=0$ and we need not worry. 
Let us summarize this estimate. It will be convenient to set $\mathcal{A}_{n}=$ $\left\{Q_{n}\right\}$ and $\mathcal{A}_{n}^{*}=\varnothing$ when $Q_{n} \in I_{1}$. We claim that

$$
\begin{aligned}
\left|T^{\varepsilon} \varphi_{n}(x)\right| \leq & C \sum_{Q \in \mathcal{A}_{n} \cup \mathcal{A}_{n}^{*}} \theta(Q) e_{Q}^{*}(x) \\
& +C \mathbf{1}_{T(n, \varepsilon)}(x) \mu\left(H_{n}\right) \operatorname{dist}\left(x, Q_{n}\right)^{-1},
\end{aligned}
$$

when $x \in E_{\infty}$ or $x \in \mathcal{C}_{m}$ for some $m \neq n$.

When $\operatorname{dist}\left(x, Q_{n}\right) \leq A^{-k_{n}+2}$, this follows from (4.99) because $\rho_{Q}(x) \geq C^{-1}$ for all $Q \in \mathcal{A}_{n} \cup \mathcal{A}_{n}^{*}$. When $\operatorname{dist}\left(x, Q_{n}\right) \geq A^{-k_{n}+2}$ and $x \in T(n, \varepsilon)$, (4.104) follows from (4.102). Otherwise we may use (4.100) (or else $T^{\varepsilon} \varphi_{n}(x)=0$ ). This completes the proof of (4.104).

There are situations where we do not want to use (4.104), because some of the $e_{Q}^{*}(x)$ are loo large. This is typically the case when $Q_{n} \in$ PLI and $x \in Q$ for one of the cubes $Q$ that compose $G_{n}$. We want to prove an alternate estimate for such cases.

For each $m \geq 1$, denote by $Q_{m}^{*}$ the cube such that

$$
\mathcal{C}_{m}=\partial\left(\frac{1}{2} B\left(Q_{m}^{*}\right)\right)
$$

Thus $Q_{m}^{*}=Q_{m}$ if $Q_{m} \in I_{1}$, and $Q_{m}^{*}$ is one of the cubes of $\mathcal{A}_{m}$ when $Q_{m} \in$ PLI.

Lemma 4.105. Let $Q \in \mathrm{BLI}$, and suppose that $x \in E_{\infty} \cap Q$ or that

$$
x \in \mathcal{C}_{m}, Q_{m}^{*} \subset Q, \text { and } \varepsilon \geq \frac{1}{5} A^{-k_{m}} .
$$

Then

$$
\left|T^{\varepsilon}\left(\mathbf{1}_{Q} f d \mu\right)(x)\right| \leq C+h_{Q}^{*}(x) .
$$

Set $a=T^{\varepsilon}\left(\mathbf{1}_{Q} f d \mu\right)(x)$. If $\varepsilon \geq A^{-k(Q)+1}$, then $a=0$. So let us assume that $\varepsilon<A^{-k(Q)+1}$. Write $\mathbf{1}_{Q}=\mathbf{1}_{E}-\mathbf{1}_{E \backslash Q}$ to get that

$$
a=T^{\varepsilon}(f d \mu)(x)-\int_{(E \backslash Q) \backslash B(x, \varepsilon)} \frac{f(y) d \mu(y)}{x-y} .
$$

Because of (4.97) and (4.98), we have that

$$
\left|T^{\varepsilon}(f d \mu)(x)\right| \leq C
$$


and

$$
\begin{aligned}
& \left|\int_{(E \backslash Q) \backslash B\left(x, A^{-k(Q)+1}\right)} \frac{f(y) d \mu(y)}{x-y}\right| \\
& =\left|T^{A^{-k(Q)+1}}(f d \mu)(x)\right| \leq C .
\end{aligned}
$$

For (4.109) we are also using (4.106) when $x \in \mathcal{C}_{m}$. Since $\varepsilon<A^{-k(Q)+1}$ we deduce from (4.108), (4.109) and (4.110) that

$$
\begin{aligned}
|a| & \leq C+\int_{(E \backslash Q) \cap B\left(x, A^{-k(Q)+1}\right)} \frac{d \mu(y)}{|x-y|} \\
& \leq C+h_{Q}^{*}(x)+\int_{(E \backslash 2 Q) \cap B\left(x, A^{-k(Q)+1}\right)} \frac{d \mu(y)}{|x-y|}
\end{aligned}
$$

(by (4.80)). Observe that dist $(x, E \backslash 2 Q) \geq A^{-k(Q)} / 2$. When $x \in Q$, this comes from the definition (4.79) of $2 Q$; if $x \in \mathcal{C}_{m}$ and $Q_{m}^{*}=Q$, then we even have that $\operatorname{dist}(x, E \backslash Q) \geq A^{-k(Q)} / 2$, by (3.8); if $x \in \mathcal{C}_{m}$ and $Q_{m}^{*}$ is strictly smaller than $Q$, then $\operatorname{dist}(x, Q) \leq C_{0} A^{-k_{m}}<A^{-k(Q)} / 2$ and dist $(x, E \backslash 2 Q) \geq A^{-k(Q)} / 2$ as well. Now the last integral in (4.111) is less than $C A^{k(Q)} \mu\left(B\left(x, A^{-k(Q)+1}\right)\right)$, which is less than $C$ by $(4.95)$ or (4.96). This proves the lemma.

We are now ready to prove Proposition 4.81. Let $x \in E_{\infty}$ and $\varepsilon>0$ be given. Obviously,

$$
\left|T^{\varepsilon}(g d \nu)(x)\right| \leq\left|T^{\varepsilon}(f d \mu)(x)\right|+\sum_{n \geq 1}\left|T^{\varepsilon} \varphi_{n}(x)\right|
$$

The first term $\left|T^{\varepsilon}(f d \mu)(x)\right|$ is less than $C$, by (4.97). Next let us take care of the set $\mathcal{N}_{1}$ of integers $n$ such that $x \notin Q_{n}$. We apply (4.104) to each $n \in \mathcal{N}_{1}$, sum, and get that

$$
\begin{aligned}
\sum_{n \in \mathcal{N}_{1}}\left|T^{\varepsilon} \varphi_{n}(x)\right| \leq & C \sum_{Q \in I_{1} \cup \operatorname{LI} \cup \mathrm{BLI}} \theta(Q) \mathbf{1}_{E \backslash Q}(x) e_{Q}^{*}(x) \\
& +C \sum_{n \in \mathcal{N}_{1}} \mathbf{1}_{T(n, \varepsilon)}(x) \mu\left(H_{n}\right) \operatorname{dist}\left(x, Q_{n}\right)^{-1}
\end{aligned}
$$

because the sets $\mathcal{A}_{n} \cup \mathcal{A}_{n}^{*}$ are all disjoint and contained in $I_{1} \cup \mathrm{LI} \cup \mathrm{BLI}$. The first sum is less than the right-hand side of (4.82); the second one is controlled as follows. 
Lemma 4.114. For each $x \in \mathbb{C}$,

$$
\sum_{n \geq 1} \mathbf{1}_{T(n, \varepsilon)}(x) \mu\left(H_{n}\right) \operatorname{dist}\left(x, Q_{n}\right)^{-1} \leq C .
$$

If $x \in T(n, \varepsilon)$, then $\operatorname{dist}\left(x, Q_{n}\right) \geq A^{-k_{n}+2}$, which is much larger than diam $\left(Q_{n} \cup \mathcal{C}_{n}\right)$. (See (4.103) for the definition of $T(n, \varepsilon)$.) Then (4.101) implies that dist $\left(x, Q_{n}\right) \geq \varepsilon / 2$ and $Q_{n} \subset B(x, 2 \varepsilon)$. The lemma follows from (4.70).

So we control the set $\mathcal{N}_{1}$. Now let $\mathcal{N}_{2}$ be the set of integers such that $x \in Q_{n}$. Since $x \in E_{\infty}$, the only possibility is that $Q_{n} \in$ PLI and $x \in G_{n}$. Let $\widetilde{Q}$ denote the cube of $\mathcal{A}_{n}^{*}$ that contains $x$. Let us use (4.72) to estimate $T^{\varepsilon} \varphi_{n}(x)$. First,

$$
\left|T^{\varepsilon}\left(\mathbf{1}_{H_{n}} \rho_{n-1} f d \mu\right)(x)\right| \leq C \sum_{Q \in \mathcal{A}_{n}} \mathbf{1}_{E \backslash Q}(x) e_{Q}^{*}(x)
$$

by definition (4.78) of $e_{Q}^{*}$ and the fact that $\rho_{Q}(x) \geq C^{-1}$ for $Q \in \mathcal{A}_{n}$. Similarly,

$$
\left|T^{\varepsilon}\left(\theta_{n} \mathbf{1}_{G_{n} \backslash \widetilde{Q}} \rho_{n-1} f d \mu\right)(x)\right| \leq C \sum_{\substack{Q \in \mathcal{A}_{n}^{*} \\ Q \neq \widetilde{Q}}} \theta(Q) \mathbf{1}_{E \backslash Q}(x) e_{Q}^{*}(x)
$$

Next observe that $\rho_{n-1}$ is constant on $\widetilde{Q}$; thus we may apply Lemma 4.105 to $\widetilde{Q}$ and get that

$$
\begin{aligned}
& \left|T^{\varepsilon}\left(\theta_{n} \mathbf{1}_{\widetilde{Q}} \rho_{n-1} f d \mu\right)(x)\right| \\
& \leq C \theta_{n} \rho_{n-1}(x)+C \theta(\widetilde{Q}) \mathbf{1}_{\widetilde{Q}}(x) h_{\widetilde{Q}}^{*}(x) .
\end{aligned}
$$

Finally

$$
\begin{aligned}
\left|T^{\varepsilon}\left(\alpha_{n} d \nu_{n}\right)(x)\right| & \leq C \mu\left(H_{n}\right) \operatorname{dist}\left(x, \mathcal{C}_{n}\right)^{-1} \\
& \leq C \sum_{Q \in \mathcal{A}_{n}} \mathbf{1}_{E \backslash Q}(x) e_{Q}^{*}(x)
\end{aligned}
$$

by (4.73) and the fact that $|x-y| \leq C \operatorname{dist}\left(x, \mathcal{C}_{n}\right)$ for all $y \in H_{n}$, by Lemma 4.43. 
We get an estimate for $\left|T^{\varepsilon} \varphi_{n}(x)\right|$ by summming (4.115), (4.116), (4.117) and (4.118). When we sum this over all $n \in \mathcal{N}_{2}$, we get terms that are obviously controlled by the right-hand side of (4.82), plus $C \sum_{n} \theta_{n} \rho_{n-1}(x)$, which comes from (4.117). Now (4.27) says that $\rho_{n}(x)=\left(1-\theta_{n}\right) \rho_{n-1}(x)$ because $x \in G_{n}$. Thus $\theta_{n} \rho_{n-1}(x)=$ $\rho_{n-1}(x)-\rho_{n}(x)$, and $\sum_{n} \theta_{n} \rho_{n-1}(x) \leq 1$. This completes our proof of Proposition 4.81.

We may now turn to the proof of Proposition 4.85. Let $x \in \mathcal{C}_{m}$ and $\varepsilon>0$ be given. Let us first observe that we can reduce to the case when $\varepsilon \geq \varepsilon_{0}=A^{-k_{m}} / 4$. Indeed, if $\varepsilon<\varepsilon_{0}$,

$$
\begin{aligned}
\mid T^{\varepsilon}(g d \nu)(x)- & T^{\varepsilon_{0}}(g d \nu)(x) \mid \\
& =\left|\int_{B\left(x, \varepsilon_{0}\right) \backslash B(x, \varepsilon)} \frac{g(y) d \nu(y)}{x-y}\right| \leq C,
\end{aligned}
$$

because Lemma 4.43 tells us that on the range $\varepsilon<|x-y| \leq \varepsilon_{0}, g d \nu$ is reduced to $\alpha_{m} d \nu_{m}$; the last inequality in (4.119) then follows from the good boundedness properties of the Cauchy integral on a circle $\mathcal{C}_{m}$, plus the fact that the density $\alpha_{m} \mu\left(H_{m}\right) H^{1}\left(\mathcal{C}_{m}\right)^{-1}$ of $\alpha_{m} d \nu_{m}$ against Hausdorff measure is at most $C$ by (4.73) and (4.13).

So let us assume that $\varepsilon \geq \varepsilon_{0}$ from now on. Then $\left|T^{\varepsilon}(f d \mu)(x)\right| \leq C$ by (4.98), and

$$
\left|T^{\varepsilon}(g d \nu)(x)\right| \leq C+\sum_{n \geq 1}\left|T^{\varepsilon} \varphi_{n}(x)\right|
$$

Let $\mathcal{N}_{1}$ be the set of integers $n$ such that $m \in J(Q)$ for all $Q \in \mathcal{A}_{n} \cup \mathcal{A}_{n}^{*}$. (See just after Proposition 4.81 for the definition of $J(Q)$.) The cubes $Q_{n}, n \in \mathcal{N}_{1}$, can be treated as before: we use (4.104) for each $n \in \mathcal{N}_{1}$, and when we sum we get

$$
\begin{aligned}
\sum_{n \in \mathcal{N}_{1}}\left|T^{\varepsilon} \varphi_{n}(x)\right| \leq & C \sum_{n \in \mathcal{N}_{1}} \sum_{Q \in \mathcal{A}_{n} \cup \mathcal{A}_{n}^{*}} \theta(Q) e_{Q}^{*}(x) \\
& +C \sum_{n \in \mathcal{N}_{1}} \mathbf{1}_{T(n, \varepsilon)}(x) \mu\left(H_{n}\right) \operatorname{dist}\left(x, Q_{n}\right)^{-1}
\end{aligned}
$$

The first term is controlled by the right-hand side of (4.86) because $\widetilde{e}_{Q}(x)=e_{Q}^{*}(x)$ for all the cubes $Q$ that show up (by the definition of $\mathcal{N}_{1}$ ). The second sum is less than $C$, by Lemma 4.114 . 
Thus we are left with the set $\mathcal{N}_{2}$ of integers $n$ for which $m \notin J(Q)$ for some $Q \in \mathcal{A}_{n} \cup \mathcal{A}_{n}^{*}$.

Lemma 4.122. If $n \in \mathcal{N}_{2}$, then either $m=n$ or else $Q_{n} \in$ PLI and $Q_{m} \subset G_{n}$.

Proof. Let $n \in \mathcal{N}_{2}$. Since $m \notin J(Q)$ for some $Q \in \mathcal{A}_{n} \cup \mathcal{A}_{n}^{*}, Q_{m}$ and $Q_{n}$ must meet. Suppose $m \neq n$. Then either $Q_{n}$ is strictly contained in $Q_{m}$ or $Q_{m}$ is strictly contained in $Q_{n}$. Since the cubes in $I_{1} \cup \mathrm{LI}$ are disjoint by (4.11), the first case implies that $Q_{m} \in \mathrm{PLI}$ and $Q_{n} \subset G_{m}$. But then $m \in J(Q)$ for all $Q \in \mathcal{A}_{n} \cup \mathcal{A}_{n}^{*}$, which is not possible since $n \in \mathcal{N}_{2}$. Similarly, the second case implies that $Q_{n} \in$ PLI and $Q_{m} \subset$ $G_{n}$, which is what we want.

Now let $n \in \mathcal{N}_{2}, n \neq m$, be given, and denote by $\widetilde{Q}$ the cube of $\mathcal{A}_{n}^{*}$ that contains $Q_{m}$. We want to use (4.72) and proceed as in (4.115-118) to estimate $T^{\varepsilon} \varphi_{n}(x)$. First let $\varphi=-\mathbf{1}_{H_{n}} \rho_{n-1} f d \mu-$ $\theta_{n} \mathbf{1}_{G_{n} \backslash \widetilde{Q}} \rho_{n-1} f d \mu$. Then a brutal estimate gives that

$$
\left|T^{\varepsilon} \varphi(x)\right| \leq C \sum_{\substack{Q \in \mathcal{A}_{n} \cup \mathcal{A}_{n}^{*} \\ Q \neq \widetilde{Q}}} \theta(Q) e_{Q}^{*}(x)
$$

because $\rho_{Q}(x) \geq C^{-1}$ for $Q \in \mathcal{A}_{n} \cup \mathcal{A}_{n}^{*}$. Notice that if $Q \in \mathcal{A}_{n} \cup \mathcal{A}_{n}^{*} \backslash$ $\{\widetilde{Q}\}, Q$ does not meet $Q_{m}$ and hence $m \in J(Q)$ and $e_{Q}^{*}(x)=\widetilde{e}_{Q}(x)$.

Denote by $\rho_{n}^{*}$ the constant value of $\rho_{n-1}$ on $\widetilde{Q}$. Lemma 4.105 (applied to $\widetilde{Q}$ ) gives that

$$
\left|T^{\varepsilon}\left(\theta_{n} \mathbf{1}_{\widetilde{Q}} \rho_{n-1} f d \mu\right)(x)\right| \leq C \theta_{n} \rho_{n}^{*}+C \theta(\widetilde{Q}) h_{\widetilde{Q}}^{*}(x) .
$$

Notice also that $h_{\widetilde{Q}}^{*}(x)=\widetilde{h}_{\widetilde{Q}}(x)$, because $Q_{m} \subset \widetilde{Q}$. The last piece from (4.72) is treated as before

$$
\left|T^{\varepsilon}\left(\alpha_{n} d \nu_{n}\right)(x)\right| \leq C \mu\left(H_{n}\right) \operatorname{dist}\left(x, \mathcal{C}_{n}\right)^{-1} \leq C \sum_{Q \in \mathcal{A}_{n}} e_{Q}^{*}(x)
$$

because $\operatorname{dist}\left(x, \mathcal{C}_{n}\right) \geq \operatorname{dist}\left(\mathcal{C}_{m}, \mathcal{C}_{n}\right) \geq A^{-k_{n}} / 4 \geq C^{-1}|x-y|$ for all $y \in H_{n}$, by (4.45). When we sum (4.123), (4.124) and (4.125) over all $n \in \mathcal{N}_{2} \backslash\{m\}$, we get terms that are less than the right-hand side 
of (4.86), plus $C \sum_{n} \theta_{n} \rho_{n}^{*}$ that comes from (4.124). Pick any point $x_{0} \in Q_{m}$. Then $\rho_{n}^{*}=\rho_{n-1}\left(x_{0}\right)$ for all $n \in \mathcal{N}_{2} \backslash\{m\}$, and

$$
\sum_{n} \theta_{n} \rho_{n}^{*}=\sum_{n} \theta_{n} \rho_{n-1}\left(x_{0}\right)=\sum_{n}\left(\rho_{n-1}\left(x_{0}\right)-\rho_{n}\left(x_{0}\right)\right) \leq 1,
$$

by the same argument using (4.27) as before.

The proof of Proposition 4.85 will be complete as soon as we estimate $T^{\varepsilon} \varphi_{m}(x)$. The good properties of the Cauchy integral on $\mathcal{C}_{m}$, plus the fact that the density $\left|\alpha_{m}\right|\left\|\nu_{m}\right\| H^{1}\left(\mathcal{C}_{m}\right)^{-1}$ is less than $C$ by (4.73) and (4.13), yield

$$
\left|T^{\varepsilon}\left(\alpha_{m} d \nu_{m}\right)(x)\right| \leq C
$$

Next let $Q_{m}^{*}$ denote the cube such that $\mathcal{C}_{m}=\partial\left(B\left(Q_{m}^{*}\right) / 2\right.$ ). (Thus $Q_{m}^{*}=Q_{m}$ if $Q_{m} \in I_{1}$ and $Q_{m}^{*} \in \mathcal{A}_{m}$ otherwise.) Let us check that

$$
\left|T^{\varepsilon}\left(\mathbf{1}_{Q_{m}^{*}} f d \mu\right)(x)\right| \leq C .
$$

Indeed, $T^{\varepsilon}\left(\mathbf{1}_{Q_{m}^{*}} f d \mu\right)(x)=0$ if $\varepsilon>\operatorname{diam}\left(\mathcal{C}_{m} \cup Q_{m}^{*}\right)$, and so we are reduced to the case when $A^{-k_{m}} / 4 \leq \varepsilon \leq A^{-k_{m}+1}$, say. (Recall that we supposed that $\varepsilon \geq A^{-k_{m}} / 4$ using (4.119). A simple computation gives that $\left|T^{\varepsilon}\left(\mathbf{1}_{Q_{m}^{*}} f d \mu\right)(x)\right| \leq C A^{k_{m}} \mu\left(Q_{m}^{*}\right) \leq C$, by (4.13).

If $Q_{m} \in I_{1}$, we can sum (4.126) and (4.127) (multiplied by the constant value of $\rho_{m-1}$ on $\left.Q_{m}\right)$ to get that $\left|T^{\varepsilon} \varphi_{m}(x)\right| \leq C$. Otherwise, we also have to add

$$
\begin{array}{r}
\left|T^{\varepsilon}\left(\mathbf{1}_{H_{m} \backslash Q_{m}^{*}} \rho_{m-1} f d \mu+\theta_{m} \mathbf{1}_{G_{m}} \rho_{m-1} f d \mu\right)(x)\right| \\
\leq C \mu\left(Q_{m}\right) \operatorname{dist}\left(x, Q_{m} \backslash Q_{m}^{*}\right)^{-1} \leq C
\end{array}
$$

by (4.13) and the fact that $\operatorname{dist}\left(x, Q_{m} \backslash Q_{m}^{*}\right) \geq \operatorname{dist}\left(\mathcal{C}_{m}, E \backslash Q_{m}\right) \geq$ $A^{-k_{m}} / 4$. This completes our proof of Proposition 4.85.

REMARK 4.129. In Propositions 4.81 and 4.85 we stated estimates for $T^{*}(g d \nu)(x)$, but our proof also gives estimates for the pieces that compose it. For each set $J \subset \mathbb{N}^{*}$ of indices, set

$$
T_{J}^{*}(x)=T^{*}(f d \mu)(x)+\sup _{\varepsilon>0}\left(\sum_{n \in J}\left|T^{\varepsilon} \varphi_{n}(x)\right|\right), \quad \text { for } x \in E_{\infty}
$$


where $\varphi_{n}=F_{n}-F_{n-1}$ is as above. If we follow the proof of Proposition 4.81 , but forget the estimates for $T^{\varepsilon} \varphi_{n}(x)$ when $n \notin J$, we get that

$$
\begin{aligned}
T_{J}^{*}(x) \leq & C+C \sum_{n \in J} \sum_{Q \in \mathcal{A}_{n} \cup \mathcal{A}_{n}^{*}} \theta(Q) \mathbf{1}_{E \backslash Q}(x) e_{Q}^{*}(x) \\
& +C \sum_{\substack{n \in J \\
Q \in \mathrm{PLI}}} \sum_{\substack{Q \in \mathcal{A}_{n}^{*} \\
Q_{n}}} \theta(Q) \mathbf{1}_{Q}(x) h_{Q}^{*}(x),
\end{aligned}
$$

for all $x \in E_{\infty}$.

There is a similar estimate when $x \in \bigcup_{m} \mathcal{C}_{m}$, but we have to be slightly careful because we do not have any good estimate for $T^{\varepsilon}(f d \mu)(x)$ when $x \in \mathcal{C}_{m}$ and $\varepsilon \ll A^{-k_{m}}$. So we set

$$
\begin{aligned}
T_{J}^{*}(x)= & \sup \left\{\left|T^{\varepsilon}(f d \mu)(x)\right|: \varepsilon \geq \frac{1}{5} A^{-k_{m}}\right\} \\
& +\sup \left\{\sum_{n \in J}\left|T^{\varepsilon} \varphi_{n}(x)\right|: \varepsilon \geq \frac{1}{5} A^{-k_{m}}\right\},
\end{aligned}
$$

when $x \in \mathcal{C}_{m}$. Then our proof of Proposition 4.85 also gives that

$$
\begin{aligned}
& T_{J}^{*}(x) \leq C+C \sum_{n \in J} \sum_{Q \in \mathcal{A}_{n} \cup \mathcal{A}_{n}^{*}} \theta(Q) \widetilde{e}_{Q}(x) \\
& +C \sum_{\substack{n \in J \\
Q_{n} \in \mathrm{PLI}}} \sum_{Q \in \mathcal{A}_{n}^{*}} \theta(Q) \widetilde{h}_{Q}(x),
\end{aligned}
$$

for $x \in \bigcup_{m} \mathcal{C}_{m}$.

5. The $L^{2}$-estimates.

Set $\mathcal{R}=I_{1} \cup \mathrm{LI} \cup \mathrm{BLI}$, and define four functions by

$$
\begin{gathered}
W_{1}=\sum_{Q \in \mathrm{BLI}} \theta(Q) \mathbf{1}_{Q \cap E_{\infty}} h_{Q}^{*}, \\
\widetilde{W}_{1}=\sum_{Q \in \mathrm{BLI}} \theta(Q) \widetilde{h}_{Q}, \\
W_{2}=\sum_{Q \in \mathcal{R}} \theta(Q) \mathbf{1}_{E_{\infty} \backslash Q} e_{Q}^{*},
\end{gathered}
$$


and

$$
\widetilde{W}_{2}=\sum_{Q \in \mathcal{R}} \theta(Q) \widetilde{e}_{Q}
$$

In this part we show that $W_{1}, \widetilde{W}_{1}, W_{2}$ and $\widetilde{W}_{2}$ lie in $L^{2}(d \nu)$; of course Theorem 2.4 will follow from this and Propositions 4.81 and 4.85.

The estimates that follow are a little unpleasant at times. It is probably a good idea to keep in mind that when $\mu$ is a nice doubling measure, we know how to prove the desired estimates. Here we shall try to keep the same general organization. A typical thing that we like to do is try to estimate scalar products in terms of the measures $\mu(Q)$ themselves, rather than quantities like $\mu(100 B(Q))$, because the $\mu(Q)$ 's are easier to sum since the cubes are often disjoint. This is why we often do not want to get "bad cubes", i.e., cubes that do not satisfy (3.14). It will often be useful to decompose bad cubes into smaller good cubes, as in the next section.

\subsection{The functions $W_{1}$ and $\widetilde{W}_{1}$.}

First we introduce a few definitions that will make it possible to estimate the functions $W_{1}$ and $\widetilde{W}_{1}$ in a unified way and save some time. Let $M_{1}$ be a large constant, to be chosen soon, and define a function $r$ on $E$ by

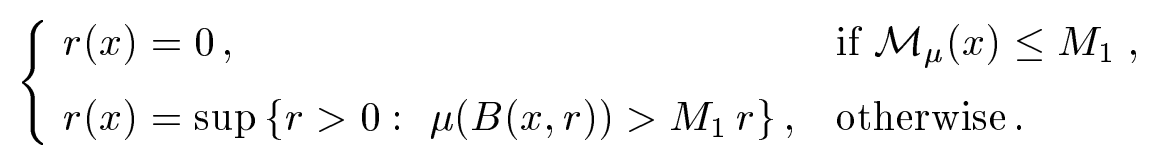

We choose $M_{1}$ so large, compared with the constants from (4.95) and (4.96), that

$$
r(x)=0, \quad \text { when } x \in E_{\infty},
$$

and

$$
r(x) \leq A^{-k_{n}}, \quad \text { when } x \in Q_{n} .
$$

Next set

$$
h_{Q}(x)=\mathbf{1}_{Q}(x) \int_{2 Q \backslash Q} \frac{d \mu(y)}{r(x)+|x-y|},
$$


for each $Q \in \Delta$. A rapid comparison with the definition (4.80) of $h_{Q}^{*}(x)$ shows that $h_{Q}(x)=\mathbf{1}_{Q}(x) h_{Q}^{*}(x)$ for $x \in E_{\infty}$. Set

$$
W_{3}=\sum_{Q \in \mathrm{BLI}} \theta(Q) h_{Q}
$$

Lemma 5.10. We have that

$$
\left\|W_{1}+\widetilde{W}_{1}\right\|_{L^{2}(d \nu)} \leq C\left\|W_{3}\right\|_{L^{2}(d \mu)} .
$$

It is clear that

$$
\left\|W_{1}\right\|_{L^{2}(d \nu)}^{2}=\int_{E_{\infty}} W_{1}^{2} \rho_{\infty} d \mu=\left\|W_{3}\right\|_{L^{2}\left(\rho_{\infty} d \mu\right)}^{2} \leq\left\|W_{3}\right\|_{L^{2}(d \mu)}^{2},
$$

because $d \nu=\rho_{\infty} d \mu$ on $E_{\infty}$. So it will be enough to control $\widetilde{W}_{1}$. We write that

$$
\left\|\widetilde{W}_{1}\right\|_{L^{2}(d \nu)}^{2}=\sum_{Q \in \mathrm{BLI}} \sum_{Q^{\prime} \in \mathrm{BLI}} \theta(Q) \theta\left(Q^{\prime}\right)\left\langle\widetilde{h}_{Q}, \widetilde{h}_{Q^{\prime}}\right\rangle_{\nu}
$$

with

$$
\left\langle\widetilde{h}_{Q}, \widetilde{h}_{Q^{\prime}}\right\rangle_{\nu}=\int \widetilde{h}_{Q} \widetilde{h}_{Q^{\prime}} d \nu=\sum_{n: Q_{n} \subset Q \cap Q^{\prime}} \int_{\mathcal{C}_{n}} h_{Q}^{*} h_{Q^{\prime}}^{*} d \nu_{n} .
$$

Fix $n$ such that $Q_{n} \subset Q \cap Q^{\prime}$. We claim that

$$
h_{Q}^{*}(x) \leq C h_{Q}(\widetilde{x}), \quad \text { for each } x \in \mathcal{C}_{n} \text { and each } \widetilde{x} \in H_{n} .
$$

Indeed, if $x \in \mathcal{C}_{n}, \widetilde{x} \in H_{n}$ and $y \in 2 Q \backslash Q$,

$$
r(x)+|\widetilde{x}-y| \leq A^{-k_{n}}+|\widetilde{x}-y| \leq C A^{-k_{n}}+|x-y| \leq C|x-y|,
$$

by $(5.7)$ and because $|x-y| \geq \operatorname{dist}\left(\mathcal{C}_{n}, E \backslash Q\right) \geq \operatorname{dist}\left(\mathcal{C}_{n}, E \backslash Q_{n}\right) \geq$ $A^{-k_{n}} / 2$. (We used the fact that $Q_{n} \subset Q$ and (4.46).) Also, $\widetilde{x} \in Q$ because $H_{n} \subset Q_{n} \subset Q$, and (5.14) follows from (5.15) by taking inverses and integrating on $y \in 2 Q \backslash Q$. Of course the analogue of (5.14) for $Q^{\prime}$ holds as well, and so

$$
\int_{\mathcal{C}_{n}} h_{Q}^{*}(x) h_{Q^{\prime}}^{*}(x) d \nu_{n}(x) \leq C \int_{H_{n}} h_{Q}(\widetilde{x}) h_{Q^{\prime}}(\widetilde{x}) d \mu(\widetilde{x})
$$


because $\left\|\nu_{n}\right\| \leq C \mu\left(H_{n}\right)$, by (4.73). Now we use the fact that the sets $H_{n}$ are disjoint subsets of $Q$ (by (4.69)) to sum and get that

$$
\left\langle\widetilde{h}_{Q}, \widetilde{h}_{Q^{\prime}}\right\rangle_{\nu} \leq C\left\langle h_{Q}, h_{Q^{\prime}}\right\rangle
$$

where

$$
\left\langle h_{Q}, h_{Q^{\prime}}\right\rangle=\int_{Q \cap Q^{\prime}} h_{Q} h_{Q^{\prime}} d \mu
$$

Lemma 5.10 follows at once from this and (5.12) because

$$
\left\|W_{3}\right\|_{L^{2}(d \mu)}^{2}=\sum_{Q \in \mathrm{BLI}} \sum_{Q^{\prime} \in \mathrm{BLI}} \theta(Q) \theta\left(Q^{\prime}\right)\left\langle h_{Q}, h_{Q^{\prime}}\right\rangle
$$

Next we want to estimate the functions $h_{Q}$. Let us first check that

$$
\int_{B\left(x, r_{2}\right) \backslash B\left(x, r_{1}\right)} \frac{d \mu(y)}{r(x)+|x-y|} \leq C\left(1+\log \frac{r_{2}}{r_{1}}\right),
$$

for all $x \in E$ and $r_{2}>r_{1}>0$. This is easy to prove. First observe that

$$
\int_{B(x, r)} \frac{d \mu(y)}{r(x)+|x-y|} \leq \frac{\mu(B(x, r(x)))}{r(x)} \leq C
$$

by the definition of $r(x)$. So we may as well assume that $r_{1} \geq r(x)$. Set

$$
A_{k}=\left\{y \in E: 2^{k} r_{1}<|x-y| \leq 2^{k+1} r_{1}\right\} \text {, }
$$

for all $k \geq 0$. Then $\mu\left(A_{k}\right) \leq C 2^{k} r_{1}$ because $r(x) \leq r_{1}$, and hence

$$
\int_{A_{k}} \frac{d \mu(y)}{r(x)+|x-y|} \leq C
$$

for all $k$. Our claim (5.20) follows from this, because the domain of integration in (5.20) can be covered by less than $C\left(1+\log \left(r_{2} / r_{1}\right)\right)$ domains $A_{k}$.

Lemma 5.22. For each $Q \in \Delta$ and $\lambda>0$,

$$
\mu\left(\left\{x \in Q: h_{Q}(x) \geq \lambda\right\}\right) \leq C e^{-c \lambda} \mu(90 B(Q)),
$$


where $c$ is some small positive constant.

For each $x \in Q$, set $d(x)=\operatorname{dist}(x, E \backslash Q)$. Then $d(x) \leq|x-y| \leq$ $100 C_{0} A^{-k(Q)}$, say, for all $y \in 2 Q \backslash Q$. Thus (5.20) tells us that

$$
h_{Q}(x) \leq C\left(1+\log \left(1+d(x)^{-1} A^{k(Q)}\right)\right) .
$$

The lemma will not be difficult to deduce from this and the small boundary property of our cubes. Set

$$
\begin{aligned}
N_{l}(Q)= & \left\{x \in E \backslash Q: \operatorname{dist}(x, Q)<A^{-k(Q)-l}\right\} \\
& \cup\left\{x \in Q: \operatorname{dist}(x, E \backslash Q)<A^{-k(Q)-l}\right\} .
\end{aligned}
$$

This is the same set as in (3.10-12); (3.13) and our choice of $A=C C_{0}^{100}$ in (4.1) give that

$$
\mu\left(N_{l}(Q)\right) \leq C_{0}^{-93 l} \mu(90 B(Q)),
$$

for all $Q \in \Delta$ and $l \geq 0$. Of course the power 93 will not be needed precisely here; this is just what we get by being too cautious.

Now let $\lambda>0$ be reasonably large (otherwise, there is nothing to prove). From (5.24) we deduce that if $h_{Q}(x) \geq \lambda$, then $d(x) \leq$ $C A^{-k(Q)} e^{-\lambda / C}$, and hence $x \in N_{l}(Q)$ for some $l \geq \lambda / C^{\prime}$. The lemma follows from this and (5.26).

We shall find Lemma 5.22 more pleasant to use when $Q$ is a good cube, i.e., when

$$
\mu(100 B(Q)) \leq C_{0} \mu(B(Q)) \leq C_{0} \mu(Q)
$$

(the second inequality is automatic, since $Q$ always contains $E \cap B(Q)$ ). Otherwise, we shall find it useful to cut $Q$ into a bunch of maximal good cubes, and then apply Lemma 5.22 to each of them separately.

For each $Q \in \Delta$, we denote by $\mathcal{S}(Q)$ the set of maximal good cubes contained in $Q$. Thus, if $Q$ already satisfies (5.27), then $\mathcal{S}(Q)$ is just composed of $Q$ itself. Obviously the cubes of $\mathcal{S}(Q)$ are disjoint. What is more interesting is that they almost cover $Q$.

Lemma 5.28. For each $Q \in \Delta$,

$$
\mu\left(Q \backslash \bigcup_{R \in \mathcal{S}(Q)} R\right)=0 .
$$


To prove the lemma we intend to use the property (3.16) of our cubes: if $Q$ is a bad cube (i.e., if (5.27) does not hold), then $\mu(100 B(Q))$ $\leq C_{0}^{-l} \mu\left(100^{l+1} B(Q)\right)$ for all integers $l$ such that $100^{l} \leq C_{0}$. We want to apply this with $l=l_{0}$, where $l_{0}$ is the largest integer such that $100^{l+1} \leq C_{0}$. We get that

$$
\mu(100 B(Q)) \leq C_{0}^{-l_{0}} \mu\left(C_{0} B(Q)\right), \quad \text { when } Q \text { is a bad cube }
$$

Recall that we decided to take $A=C C_{0}^{100}$, where $C$ is an absolute constant, and that we still have the right to choose $C_{0}$ as large as we want. Since $C_{0}^{-l_{0}}$ decreases faster than any given power of $C_{0}$, we can choose $C_{0}$ so large that $C_{0}^{-l_{0}}<A^{-10}$, say. Then (5.29) implies that

$$
\mu(100 B(Q)) \leq A^{-10} \mu\left(C_{0} B(Q)\right) \leq A^{-10} \mu(100 B(\widehat{Q})),
$$

whenever $Q$ is a bad cube, and where $\widehat{Q}$ still denotes the parent of $Q$. Let us derive a consequence of (5.30) and then return to Lemma 5.28.

Lemma 5.31. Let $Q \in \Delta$, and let $R \subset Q$ be a cube such that all the intermediate cubes $S, R \varsubsetneqq S \varsubsetneqq Q$, are bad. Then

$$
\mu(100 B(R)) \leq A^{-10(k(R)-k(Q)-1)} \mu(100 B(Q)) .
$$

When $R=Q$ and when $R$ is a child of $Q,(5.32)$ is trivially true. Otherwise, we even have that

$$
\mu(100 B(R)) \leq \mu(100 B(\widehat{R})) \leq A^{-10(k(R)-k(Q)-1)} \mu(100 B(Q))
$$

by repeated applications of (5.30). This proves Lemma 5.31.

Return to Lemma 5.28. Let $Q \in \Delta$ be given and, for each $k>k(Q)$, denote by $Z_{k}$ the set of cubes $R \subset Q$ of generation $k$ which are not contained in any cube of $\mathcal{S}(Q)$. Also let $U_{k}$ be the union of the cubes of $Z_{k}$. Thus

$$
Q \backslash \bigcup_{R \in \mathcal{S}(Q)} R \subset U_{k}, \quad \text { for all } k>k(Q) \text {. }
$$

Note that Lemma 5.31 applies to each cube of $Z_{k}$, and so

$$
\mu(R) \leq A^{-10(k-k(Q)-1)} \mu(100 B(Q)),
$$


for all $R \in Z_{k}$.

On the other hand, the balls $B(R), R \in Z_{k}$, are disjoint by (3.9), have radii greater or equal than $A^{-k}$, and are all contained in a ball of radius $100 C_{0}$, say. Thus $Z_{k}$ has at most $C A^{2 k}$ elements. Then (5.35) implies that $\mu\left(U_{k}\right) \leq C A^{10(k(Q)+1)} A^{-8 k}$ and hence that $\mu\left(U_{k}\right)$ tends to zero when $k \longrightarrow+\infty$. Lemma 5.28 follows from this and (5.34).

Lemma 5.36. If $Q \in \mathrm{BLI}$ and $R \in \mathcal{S}(Q)$,

$$
h_{Q}(x) \leq h_{R}(x)+C,
$$

for all $x \in R$.

Let $Q \in \mathrm{BLI}, R \in \mathcal{S}(Q)$ and $x \in R$ be given. For each integer $k$ such that $k(Q) \leq k \leq k(R)$, let $R_{k}$ denote the cube of $\Delta_{k}$ that contains $R$. Also set $D_{k}=2 R_{k} \backslash 2 R_{k+1}$ for $k(Q) \leq k<k(R)$. Then

$$
\begin{aligned}
h_{Q}(x) & \leq h_{R}(x)+\int_{2 Q \backslash 2 R} \frac{d \mu(y)}{r(x)+|x-y|} \\
& \leq h_{R}(x)+\sum_{k=k(Q)}^{k(R)-1} \int_{D_{k}} \frac{d \mu(y)}{r(x)+|x-y|}
\end{aligned}
$$

(compare with the definition (5.8)). Now

$$
\mu\left(D_{k}\right) \leq \mu\left(100 B\left(R_{k}\right)\right) \leq A^{-10(k-k(Q)-1)} \mu(100 B(Q))
$$

by Lemma 5.31 , and $|x-y| \geq A^{-k-1}$ on $D_{k}$, because $x \in R_{k+1}$ and by definition (4.79) of $2 R_{k+1}$. Then

$$
\int_{D_{k}} \frac{d \mu(y)}{r(x)+|x-y|} \leq C A^{-9(k-k(Q))} A^{k(Q)} \mu(100 B(Q))
$$

and (5.37) follows from (5.38) together with our assumption that $Q \in$ BLI and the density estimate (4.13) (applied to $\widehat{Q}$ ). This proves the lemma.

We are now ready to estimate scalar products. Fix a cube $Q_{0} \in$ BLI, and first consider cubes in the set

$$
\begin{aligned}
\mathcal{B}_{1}\left(Q_{0}\right)=\{Q \in \mathrm{BLI}: & Q \subset Q_{0} \text { and } Q \text { is not strictly } \\
& \text { contained in any cube } \left.R \in S\left(Q_{0}\right)\right\} .
\end{aligned}
$$


Note that if $Q \in \mathcal{B}_{1}\left(Q_{0}\right)$ and $R \in \mathcal{S}(Q)$, then $R \in \mathcal{S}\left(Q_{0}\right)$ as well. Then

$$
\begin{aligned}
\left\langle h_{Q}, h_{Q_{0}}\right\rangle & =\int_{Q} h_{Q} h_{Q_{0}} d \mu \\
& =\sum_{R \in \mathcal{S}(Q)} \int_{R} h_{Q} h_{Q_{0}} d \mu \\
& \leq \sum_{R \in \mathcal{S}(Q)} \int_{R}\left(h_{R}+C\right)^{2} d \mu
\end{aligned}
$$

by the definition (5.18) of the scalar product, Lemma 5.28, and Lemma 5.36 applied to $Q$ and to $Q_{0}$. Next,

$$
\int_{R}\left(h_{R}+C\right)^{2} d \mu \leq C \mu(90 B(R)) \leq C \mu(R),
$$

for each $R \in \mathcal{S}(Q)$, by Lemma 5.22 and because $R$ is a good cube. Thus (5.42) yields

$$
\left\langle h_{Q}, h_{Q_{0}}\right\rangle \leq C \sum_{R \in \mathcal{S}(Q)} \mu(R)=C \mu(Q), \quad \text { for all } Q \in \mathcal{B}_{1}\left(Q_{0}\right) .
$$

Now suppose that $Q \in \mathcal{B}_{2}\left(Q_{0}\right)$, where

$$
\mathcal{B}_{2}\left(Q_{0}\right)=\left\{Q \in \mathrm{BLI}: Q \subset Q_{0}\right. \text { and there is }
$$

$$
\text { a cube } \left.R \in \mathcal{S}\left(Q_{0}\right) \text { such that } Q \varsubsetneqq R\right\} \text {. }
$$

Denote by $R=R\left(Q, Q_{0}\right)$ the cube of $\mathcal{S}\left(Q_{0}\right)$ that contains $Q$. Let us first apply Lemma 5.36 to $Q_{0}$ and $R$ to get that

$$
\left\langle h_{Q}, h_{Q_{0}}\right\rangle \leq \int_{Q} h_{Q}\left(h_{R}+C\right) d \mu .
$$

Denote by $l(Q)$ the largest integer $l \geq 0$ such that $Q \subset N_{l}(R)$, where $N_{l}(R)$ is as in (5.25). When $Q$ is not even contained in $N_{0}(R)$, still take $l(Q)=0$.

Lemma 5.47. We have that

$$
h_{R}(x) \leq h_{Q}(x)+C(1+l(Q)), \quad \text { on } Q .
$$


By definitions,

$$
h_{R}(x) \leq h_{Q}(x)+\int_{D} \frac{d \mu(y)}{r(x)+|x-y|},
$$

with $D=2 R \backslash(2 Q \cup R)$. We claim that

$$
C^{-1} A^{-k(R)-l(Q)} \leq|x-y| \leq C A^{-k(R)}, \quad \text { for } x \in Q \text { and } y \in D .
$$

The second inequality is easy, because $x$ and $y$ both lie in $2 R$. We also have that $|x-y| \geq A^{-k(Q)}$ because $x \in Q$ and $y \in E \backslash 2 Q$. This proves the first inequality when $A^{-k(R)-l(Q)-2} \leq A^{-k(Q)}$, say. So let us assume that $A^{-k(R)-l(Q)-2}>A^{-k(Q)}$. Since $Q$ is not contained in $N_{l(Q)+1}(R)$, we can find $x_{0} \in Q$ such that dist $\left(x_{0}, E \backslash R\right) \geq A^{-k(R)-l(Q)-1}$. Then $|x-y| \geq \operatorname{dist}(x, E \backslash R) \geq A^{-k(R)-l(Q)-1}-\operatorname{diam} Q \geq A^{-k(R)-l(Q)-1} / 2$, because we are in the case when $A^{-k(Q)} \leq A^{-k(R)-l(Q)-2}$. This proves our claim (5.50).

The lemma follows at once from (5.50), (5.49), and (5.20).

From (5.46) and Lemma 5.47 we deduce that

$$
\begin{aligned}
\left\langle h_{Q}, h_{Q_{0}}\right\rangle & \leq \int_{Q} h_{Q}\left(h_{Q}+C+C l(Q)\right) d \mu \\
& \leq C(1+l(Q)) \int_{Q} h_{Q}\left(h_{Q}+1\right) d \mu .
\end{aligned}
$$

Next we use Lemma 5.28 to decompose $Q$ into maximal good cubes $S$, $S \in \mathcal{S}(Q)$, and then Lemma 5.36 to replace $h_{Q}$ with $h_{S}+C$. This gives

$$
\begin{aligned}
\int_{Q} h_{Q}\left(h_{Q}+1\right) d \mu & =\sum_{S \in \mathcal{S}(Q)} \int_{S} h_{Q}\left(h_{Q}+1\right) d \mu \\
& \leq \sum_{S \in \mathcal{S}(Q)} \int_{S}\left(h_{S}+C\right)^{2} d \mu \\
& \leq C \sum_{S \in \mathcal{S}(Q)} \mu(90 B(S)) \\
& \leq C \sum_{S \in \mathcal{S}(Q)} \mu(S) \\
& =C \mu(Q)
\end{aligned}
$$


by Lemma 5.22 , the goodness of the cubes $S$, and their disjointness. Because of (5.51), this yields

$$
\left\langle h_{Q}, h_{Q_{0}}\right\rangle \leq C(1+l(Q)) \mu(Q), \quad \text { for all } Q \in \mathcal{B}_{2}\left(Q_{0}\right) .
$$

Now we want to sum our estimates from (5.44) and (5.53). Let us first check that

$$
\sum_{Q \in \mathcal{B}_{1}\left(Q_{0}\right)} \theta(Q)\left\langle h_{Q}, h_{Q_{0}}\right\rangle \leq C \mu\left(Q_{0}\right)
$$

Of course the contribution of $Q_{0}$ itself is at most $C \mu\left(Q_{0}\right)$, by (5.44). All the other cubes $Q \in \mathcal{B}_{1}\left(Q_{0}\right)$ lie in $\mathcal{A}_{n}^{*}$ for some $n$ such that $Q_{n} \in$ PLI and $Q_{n} \subset Q_{0}$. For each such $Q_{n}$,

$$
\sum_{Q \in \mathcal{A}_{n}^{*}} \theta(Q)\left\langle h_{Q}, h_{Q_{0}}\right\rangle \leq C \theta_{n} \mu\left(G_{n}\right) \leq C \mu\left(H_{n}\right)
$$

by (5.44) and (4.33). Now we can use the fact that the sets $H_{n}$ are disjoint (by (4.69)) and contained in $Q_{0}$ to sum over $n$ and get (5.54).

We want to prove a similar estimate for $\mathcal{B}_{2}\left(Q_{0}\right)$. Decompose $\mathcal{B}_{2}\left(Q_{0}\right)$ into the subsets $\mathcal{B}(R)=\{Q \in \mathrm{BLI}: Q \varsubsetneqq R\}, R \in \mathcal{S}\left(Q_{0}\right)$. For each $l \geq 0$, let $\mathcal{B}(R, l)$ denote the set of the cubes in $\mathcal{B}(R)$ such that $l(Q)=l$. If $l \geq 2$, each cube $Q \in \mathcal{B}(R, l)$ is contained in $N_{l}(R)$ (by the definition of $l(Q))$. In particular the centre of $B(Q)$, which indeed lies in $Q$, lies at distance less than $A^{-k(R)-l}$ from $E \backslash Q$ (compare with $(5.25))$. Since it also lies at distance greater or equal to $A^{-k(Q)}$ from $E \backslash Q$ (because $E \cap B(Q) \subset Q$ ), we get that $A^{-k(Q)} \leq A^{-k(R)-l}$, and then $\widehat{Q} \subset N_{l-2}(R)$. Moreover, by the definition of $\mathcal{B}(R), Q$ is a cube of BLI and its parent is a cube $Q_{n} \in$ PLI. We may now regroup cubes with a given parent $Q_{n}$ and get that for $l \geq 2$,

$$
\begin{aligned}
\sum_{Q \in \mathcal{B}(R, l)} \theta(Q)\left\langle h_{Q}, h_{Q_{0}}\right\rangle & \leq C(1+l) \sum_{Q \in \mathcal{B}(R, l)} \theta(Q) \mu(Q) \\
& \leq C(1+l) \sum_{\substack{Q_{n} \in \mathrm{PLI} \\
Q_{n} \subset N_{l-2}(R)}} \theta_{n} \mu\left(G_{n}\right) \\
& \leq C(1+l) \sum_{\substack{Q_{n} \in \mathrm{PLI} \\
Q_{n} \subset N_{l-2}(R)}} \mu\left(H_{n}\right) \\
& \leq C(1+l) \mu\left(N_{l-2}(R)\right) \\
& \leq C(1+l) C_{0}^{-93 l} \mu(90 B(R))
\end{aligned}
$$


by (5.53), (4.33), the disjointness of the sets $H_{n}$, and (5.26). When $l \leq 2$, we simply repeat the argument for $(5.54)$ or replace $N_{l-2}(R)$ by $R$ in (5.56), and get less than $C \mu(R) \leq C \mu(90 B(R))$.

We may now sum over $l$ and then $R \in \mathcal{S}\left(Q_{0}\right)$ to get that

$$
\sum_{Q \in \mathcal{B}_{2}\left(Q_{0}\right)} \theta(Q)\left\langle h_{Q}, h_{Q_{0}}\right\rangle \leq \sum_{R \in \mathcal{S}\left(Q_{0}\right)} \sum_{l \geq 0} C(1+l) C_{0}^{-l} \mu(90 B(R))
$$

$$
\begin{aligned}
& \leq C \sum_{R \in \mathcal{S}\left(Q_{0}\right)} \mu(90 B(R)) \\
& \leq C \sum_{R \in \mathcal{S}\left(Q_{0}\right)} \mu(R) \leq C \mu\left(Q_{0}\right)
\end{aligned}
$$

because the cubes $R \in \mathcal{S}\left(Q_{0}\right)$ are good, disjoint, and contained in $Q_{0}$. From this and (5.54) we deduce that

$$
\sum_{\substack{Q \in \mathrm{BLI} \\ Q \subset Q_{0}}} \theta(Q)\left\langle h_{Q}, h_{Q_{0}}\right\rangle \leq C \mu\left(Q_{0}\right) .
$$

Let us observe, also for future reference, that

$$
\sum_{Q \in \mathcal{R}} \theta(Q) \mu(Q) \leq C \mu(E)
$$

This follows by the same argument which was used to prove (4.51) and (4.76). Hence

$$
\begin{aligned}
\left\|W_{3}\right\|_{L^{2}(d \mu)}^{2} & =\sum_{Q_{0}} \sum_{Q} \theta\left(Q_{0}\right) \theta(Q)\left\langle h_{Q}, h_{Q_{0}}\right\rangle \\
& \leq 2 \sum_{Q_{0}} \sum_{Q \subset Q_{0}} \theta\left(Q_{0}\right) \theta(Q)\left\langle h_{Q}, h_{Q_{0}}\right\rangle \\
& \leq C \sum_{Q_{0}} \theta\left(Q_{0}\right) \mu\left(Q_{0}\right) \\
& \leq C \mu(E)
\end{aligned}
$$

by (5.9), the fact that $\left\langle h_{Q}, h_{Q_{0}}\right\rangle=0$ when $Q$ does not meet $Q_{0},(5.58)$ and (5.59). Because of Lemma 5.10, this also implies that

$$
\left\|W_{1}+\widetilde{W}_{1}\right\|_{L^{2}(d \nu)}^{2} \leq C \mu(E),
$$


and completes our study of $W_{1}$ and $\widetilde{W}_{1}$.

\subsection{The functions $e_{Q}$, the faraway piece.}

We would like to estimate the functions $W_{2}$ and $\widetilde{W}_{2}$ in a unified way, and to this effect we introduce a variant of the functions $e_{Q}^{*}$ and $\widetilde{e}_{Q}$ that will be easier to deal with. For each cube $Q \in \Delta$, define a function $e_{Q}$ on $E$ by

$$
e_{Q}(x)=\mathbf{1}_{E \backslash Q}(x) \rho_{Q}(x) \int_{Q} \frac{d \mu(y)}{|x-y|} .
$$

Set

$$
W(x)=\sum_{Q \in \mathcal{R}} \theta(Q) e_{Q}
$$

with $\mathcal{R}=I_{1} \cup \mathrm{LI} \cup \mathrm{BLI}$. Let us first check that $W$ dominates $W_{2}$ and $\widetilde{W}_{2}$. First $W_{2}(x)=W(x)$ for $x \in E_{\infty}$ (compare (5.62) with (5.3) and (4.78)). Then

$$
\left\|W_{2}\right\|_{L^{2}(d \nu)}^{2} \leq\|W\|_{L^{2}(d \mu)}^{2},
$$

because $d \nu=\rho_{\infty} d \mu$ on $E_{\infty}$. Next set

$$
\left\langle\widetilde{e}_{Q}, \widetilde{e}_{Q^{\prime}}\right\rangle_{\nu}=\int \widetilde{e}_{Q} \widetilde{e}_{Q^{\prime}} d \nu
$$

and

$$
\left\langle e_{Q}, e_{Q^{\prime}}\right\rangle=\int_{E} e_{Q} e_{Q^{\prime}} d \mu
$$

for all $Q, Q^{\prime} \in \mathcal{R}$. We claim that

$$
\left\langle\widetilde{e}_{Q}, \widetilde{e}_{Q^{\prime}}\right\rangle_{\nu} \leq C\left\langle e_{Q}, e_{Q^{\prime}}\right\rangle, \quad \text { for all } Q, Q^{\prime} \in \mathcal{R} \text {. }
$$

To prove this claim, first observe that

$$
\left\langle\widetilde{e}_{Q}, \widetilde{e}_{Q^{\prime}}\right\rangle_{\nu}=\sum_{n \in J(Q) \cap J\left(Q^{\prime}\right)} \int_{\mathcal{C}_{n}} e_{Q}^{*} e_{Q^{\prime}}^{*} d \nu_{n}
$$


where $J(Q)$ and $J\left(Q^{\prime}\right)$ are defined just after Proposition 4.81.

Let $n \in J(Q)$ be given. First observe that $H_{n} \subset E \backslash Q$ by definitions. (Recall that $H_{n}=Q_{n}$ when $Q_{n} \in I_{1}$, and $H_{n}=Q_{n} \backslash G_{n}$ when $Q_{n} \in$ PLI.) Then $\operatorname{dist}\left(\mathcal{C}_{n}, Q\right) \geq \operatorname{dist}\left(\mathcal{C}_{n}, E \backslash H_{n}\right) \geq A^{-k_{n}} / 2$ by (4.47). Thus for all choices of $\widetilde{x} \in H_{n}, x \in \mathcal{C}_{n}$ and $y \in Q$, we have that

$$
|\widetilde{x}-y| \leq C A^{-k_{n}}+|x-y| \leq C|x-y| \text {. }
$$

If we integrate over $y$ and observe that $H_{n} \subset E \backslash Q$, we get that

$$
e_{Q}^{*}(x) \leq C e_{Q}(\widetilde{x}), \quad \text { for } x \in \mathcal{C}_{n} \text { and } \widetilde{x} \in H_{n} .
$$

Of course a similar estimate for $Q^{\prime}$ holds if $n \in J\left(Q^{\prime}\right)$ as well, and

$$
\int_{\mathcal{C}_{n}} e_{Q}^{*} e_{Q^{\prime}}^{*} d \nu_{n} \leq C \int_{H_{n}} e_{Q} e_{Q^{\prime}} d \mu
$$

because $\left\|\nu_{n}\right\| \leq \mu\left(H_{n}\right)$ by (4.73). We may now use the fact that the sets $H_{n}$ are disjoint (by (4.69)) to sum (5.71) and prove our claim (5.67).

Of course a brutal expansion of $\left\|\widetilde{W}_{2}\right\|_{L^{2}(d \nu)}^{2}$ and $\|W\|_{L^{2}(d \mu)}^{2}$ from their definitions (5.4) and (5.63) now yields

$$
\left\|\widetilde{W}_{2}\right\|_{L^{2}(d \nu)}^{2} \leq C\|W\|_{L^{2}(d \mu)}^{2} .
$$

Thus it will be enough to control the function $W$.

We start with the "faraway piece"

$$
W^{\infty}=\sum_{Q \in \mathcal{R}} \theta(Q) \mathbf{1}_{E \backslash 2 Q} e_{Q}
$$

which we even split further as follows. For each integer $m \geq 0$, set

$$
e_{Q}^{m}=\mathbf{1}_{\mathcal{D}_{m}(Q)} e_{Q}
$$

where

$$
\mathcal{D}_{m}(Q)=\left\{x \in E: A^{-k(Q)+m}<\operatorname{dist}(x, Q) \leq A^{-k(Q)+m+1}\right\} .
$$

It is clear that $E \backslash 2 Q=\bigcup_{m \geq 0} \mathcal{D}_{m}(Q)$ (compare with (4.79)), and hence

$$
W^{\infty}=\sum_{m=0}^{\infty}\left(\sum_{Q \in \mathcal{R}} \theta(Q) e_{Q}^{m}\right)
$$


A brutal estimate using the definitions (5.62) and (4.77) gives that

$$
e_{Q}^{m}(x) \leq C A^{k(Q)-2 m} \mu(Q) \mathbf{1}_{\mathcal{D}_{m}(Q)} .
$$

We also have that

$$
\mu\left(\mathcal{D}_{m}(Q)\right) \leq C A^{-k(Q)+m},
$$

because $Q \in I_{1} \cup \mathrm{LI} \cup \mathrm{BLI}$ and by (4.13) (applied to $Q$ or one of its ancestors).

Next let $Q$ and $Q^{\prime}$ be two cubes of $\mathcal{R}$, and assume for definiteness that $k\left(Q^{\prime}\right) \geq k(Q)$. Then

$$
\begin{aligned}
\left\langle e_{Q}^{m}, e_{Q^{\prime}}^{m}\right\rangle & \leq C A^{k(Q)+k\left(Q^{\prime}\right)-4 m} \mu(Q) \mu\left(Q^{\prime}\right) \mu\left(\mathcal{D}_{m}\left(Q^{\prime}\right)\right) \\
& \leq C A^{k(Q)-3 m} \mu(Q) \mu\left(Q^{\prime}\right)
\end{aligned}
$$

by (5.77) and (5.78).

Denote by $\mathcal{R}_{m}(Q)$ the set of cubes $Q^{\prime} \in \mathcal{R}$ such that $\left\langle e_{Q}^{m}, e_{Q^{\prime}}^{m}\right\rangle \neq$ 0 . If $Q^{\prime} \in \mathcal{R}_{m}(Q)$, then $\mathcal{D}_{m}\left(Q^{\prime}\right)$ meets $\mathcal{D}_{m}(Q)$, and hence $Q^{\prime} \subset$ $B\left(x_{Q}, C A^{-k(Q)+m}\right)$, where $x_{Q}$ is for instance the centre of $B(Q)$. Then (4.51) and (4.76) tell us that

$$
\sum_{Q^{\prime} \in \mathcal{R}_{m}(Q)} \theta\left(Q^{\prime}\right) \mu\left(Q^{\prime}\right) \leq C A^{-k(Q)+m}
$$

Now

$$
\begin{aligned}
& \left\|\sum_{Q \in \mathcal{R}} \theta(Q) e_{Q}^{m}\right\|_{L^{2}(d \mu)}^{2} \\
& \quad \leq 2 \sum_{Q \in \mathcal{R}} \sum_{\substack{Q^{\prime} \in \mathcal{R} \\
k\left(Q^{\prime}\right) \geq k(Q)}} \theta(Q) \theta\left(Q^{\prime}\right)\left\langle e_{Q}^{m}, e_{Q^{\prime}}^{m}\right\rangle \\
& \quad \leq C \sum_{Q \in \mathcal{R}} \sum_{Q^{\prime} \in \mathcal{R}_{m}(Q)} \theta(Q) \theta\left(Q^{\prime}\right) A^{k(Q)-3 m} \mu(Q) \mu\left(Q^{\prime}\right) \\
& \quad \leq C \sum_{Q \in \mathcal{R}} \theta(Q) A^{-2 m} \mu(Q) \\
& \leq C A^{-2 m} \mu(E)
\end{aligned}
$$


by (5.79), (5.80), and (5.59). Of course (5.81) and the definition (5.76) yield

$$
\left\|W^{\infty}\right\|_{L^{2}(d \mu)}^{2} \leq C \mu(E)
$$

\subsection{Estimates for the semi-local part of $e_{Q}$.}

Now we wish to study the remaining part of the functions $e_{Q}$, i.e., the functions $e_{Q}-\mathbf{1}_{E \backslash 2 Q} e_{Q}=\mathbf{1}_{2 Q \backslash Q} e_{Q}$. Here again it will be helpful to use the almost decomposition of the domain $Q$ into maximal good cubes $R, R \in \mathcal{S}(Q)$. Thus we set

$$
g_{R}(x)=\int_{R} \frac{d \mu(y)}{|x-y|}
$$

for $x \in E \backslash R$, and then observe that

$$
\mathbf{1}_{2 Q \backslash Q} e_{Q} \leq \sum_{R \in \mathcal{S}(Q)} \mathbf{1}_{2 Q \backslash Q} g_{R}
$$

We further decompose each $\mathbf{1}_{2 Q \backslash Q} g_{R}$ into its very local part

$$
e_{Q, R}^{0}=\mathbf{1}_{2 R \backslash Q} g_{R}
$$

and its less local part

$$
e_{Q, R}=\mathbf{1}_{2 Q \backslash(2 R \cup Q)} g_{R} .
$$

Set

$$
W_{4}=\sum_{Q \in \mathcal{R}} \sum_{R \in \mathcal{S}(Q)} \theta(Q) e_{Q, R}
$$

and

$$
W_{5}=\sum_{Q \in \mathcal{R}} \sum_{R \in \mathcal{S}(Q)} \theta(Q) e_{Q, R}^{0} .
$$

From the discussion above it is clear that

$$
W-W^{\infty}=\sum_{Q \in \mathcal{R}} \theta(Q) \mathbf{1}_{2 Q \backslash Q} e_{Q} \leq W_{4}+W_{5} .
$$


In this section we want to estimate the semi-local part $W_{4}$.

Let $Q, Q^{\prime} \in \mathcal{R}$ and $R \in \mathcal{S}(Q), R^{\prime} \in \mathcal{S}\left(Q^{\prime}\right)$ be given. We want to estimate the scalar product

$$
\left\langle e_{Q, R}, e_{Q^{\prime}, R^{\prime}}\right\rangle=\int_{D\left(R, R^{\prime}\right)} g_{R} g_{R^{\prime}} d \mu
$$

where

$$
D\left(R, R^{\prime}\right)=\left(2 Q \cap 2 Q^{\prime}\right) \backslash\left(2 R \cup 2 R^{\prime} \cup Q \cup Q^{\prime}\right) .
$$

Obviously $g_{R}(x) \leq \operatorname{dist}(x, R)^{-1} \mu(R)$, and similarly for $R^{\prime}$. Thus

$$
\left\langle e_{Q, R}, e_{Q^{\prime}, R^{\prime}}\right\rangle \leq \mu(R) \mu\left(R^{\prime}\right) a\left(R, R^{\prime}\right),
$$

where

$$
a\left(R, R^{\prime}\right)=\int_{D\left(R, R^{\prime}\right)} \operatorname{dist}(x, R)^{-1} \operatorname{dist}\left(x, R^{\prime}\right)^{-1} d \mu(x) .
$$

Next we continue and cut each $a\left(R, R^{\prime}\right)$ into pieces $a_{k, l}$, as follows. For each $k \geq 0$ and $l \geq 0$, set

$$
\begin{aligned}
& U_{k}=\left\{x \in 2 Q: A^{-k(Q)-k}<\operatorname{dist}(x, R) \leq A^{1-k(Q)-k}\right\} \\
& V_{l}=\left\{x \in 2 Q^{\prime}: A^{-k\left(Q^{\prime}\right)-l}<\operatorname{dist}\left(x, R^{\prime}\right) \leq A^{1-k\left(Q^{\prime}\right)-l}\right\}
\end{aligned}
$$

and

$$
D_{k, l}=D\left(R, R^{\prime}\right) \cap U_{k} \cap V_{l} .
$$

Clearly, $2 Q \backslash Q$ is covered by the $U_{k}$ 's and the set $\{x \in E \backslash Q$ : $\operatorname{dist}(x, Q)=0\}$ which has $\mu$ measure zero by (3.13). Using a similar fact for $2 Q^{\prime} \backslash Q^{\prime}$ and the $V_{l}$ 's we see that $D\left(R, R^{\prime}\right)$ is the union of the $D_{k, l}$ 's and a set of $\mu$ measure zero. Thus

$$
a\left(R, R^{\prime}\right)=\sum_{k \geq 0} \sum_{l \geq 0} a_{k, l},
$$

where

$$
\begin{aligned}
a_{k, l} & =\int_{D_{k, l}} \operatorname{dist}(x, R)^{-1} \operatorname{dist}\left(x, R^{\prime}\right)^{-1} d \mu(x) \\
& \leq A^{k+l} A^{k(Q)} A^{k\left(Q^{\prime}\right)} \mu\left(D_{k, l}\right) .
\end{aligned}
$$


If $D_{k, l}$ is not empty, then $U_{k}$ meets $E \backslash 2 R$. Since dist $(x, R) \geq A^{-k(R)}$ on $E \backslash 2 R$, this forces $A^{-k(R)} \leq A^{1-k(Q)-k}$. Of course there is a similar condition on $l$, and so

$$
\begin{aligned}
& 0 \leq k \leq k(R)-k(Q)+1, \text { and } \\
& 0 \leq l \leq k\left(R^{\prime}\right)-k\left(Q^{\prime}\right)+1, \text { when } D_{k, l} \neq \varnothing .
\end{aligned}
$$

For $0<k \leq k(R)-k(Q)+1$, let $R_{k}$ denote the cube of generation $k(Q)+k-1$ that contains $R$, and for $k=0$ let $R_{k}=Q$. Since $R$ is a maximal good subcube of $Q$, we can apply Lemma 5.31 to $R_{k}$. Also, $U_{k} \subset 100 B\left(R_{k}\right)$ by definitions and so

$$
\mu\left(U_{k}\right) \leq \mu\left(100 B\left(R_{k}\right)\right) \leq C A^{-10 k} \mu(100 B(Q)) .
$$

Because $Q \in \mathcal{R}=I_{1} \cup \mathrm{LI} \cup \mathrm{BLI}$, we may apply (4.13) to $Q$ or its parent and get that $\mu(100 B(Q)) \leq C A^{-k(Q)}$. So

$$
\mu\left(U_{k}\right) \leq C A^{-10 k} A^{-k(Q)}, \quad \text { for } 0 \leq k \leq k(R)-k(Q)+1
$$

Of course the same argument applies to $V_{l}$ and gives that

$$
\mu\left(V_{l}\right) \leq C A^{-10 l} \mu\left(100 B\left(Q^{\prime}\right)\right) \leq C A^{-10 l} A^{-k\left(Q^{\prime}\right)},
$$

for $0 \leq l \leq k\left(R^{\prime}\right)-k\left(Q^{\prime}\right)+1$.

We want to distinguish two cases, depending on the size of

$$
\delta=\operatorname{dist}\left(R, R^{\prime}\right) \text {. }
$$

We start with the case when

$$
k(Q) \leq k\left(Q^{\prime}\right) \quad \text { and } \quad \delta \leq A^{-k\left(Q^{\prime}\right)+1} .
$$

(The first condition is just here for the sake of definiteness, but the second one is serious.) In this first case we want to refine the estimate (5.102) before we apply it. The idea will be that if $Q^{\prime}$ is somewhat smaller than $Q$, Lemma 5.31 will tell us that $\mu\left(100 B\left(Q^{\prime}\right)\right) \ll A^{-k\left(Q^{\prime}\right)}$.

Let us assume that we are in Case 1 , and that $D\left(R, R^{\prime}\right)$ is not empty. If $x \in D\left(R, R^{\prime}\right)$,

$$
\begin{aligned}
\operatorname{dist}(x, R) & \leq \operatorname{dist}\left(x, Q^{\prime}\right)+\operatorname{diam} Q^{\prime}+\operatorname{dist}\left(R^{\prime}, R\right) \\
& \leq A^{-k\left(Q^{\prime}\right)}+100 C_{0} A^{-k\left(Q^{\prime}\right)}+\delta \\
& \leq 2 A^{-k\left(Q^{\prime}\right)+1}
\end{aligned}
$$


because $R^{\prime} \subset Q^{\prime}, x \in 2 Q^{\prime}$, and by (5.104). Since $x \in D\left(R, R^{\prime}\right) \subset$ $E \backslash 2 R$, we also have that $\operatorname{dist}(x, R) \geq A^{-k(R)}$, and so (5.105) yields

$$
k\left(Q^{\prime}\right)-1 \leq k(R) .
$$

Now suppose that in addition $k\left(Q^{\prime}\right)-1 \geq k(Q)$, and denote by $\widetilde{R}$ the cube of generation $k\left(Q^{\prime}\right)-1$ that contains $R$. Then $R \subset \widetilde{R} \subset Q$, and we may apply Lemma 5.31 to $\widetilde{R}$ and get that

$$
\mu(100 B(\widetilde{R})) \leq C A^{-10\left(k\left(Q^{\prime}\right)-k(Q)\right)} \mu(100 B(Q)) .
$$

Since $D\left(R, R^{\prime}\right)$ meets $2 Q^{\prime},(5.105)$ tells us that all points of $100 B\left(Q^{\prime}\right)$ lie at distance at most $3 A^{-k\left(Q^{\prime}\right)+1}=3 A^{-k(\widetilde{R})}$ from $R$. Hence $100 B\left(Q^{\prime}\right)$ $\subset 100 B(\widetilde{R})$. We also know (from (4.13) applied to $Q$ or its parent) that $\mu(100 B(Q)) \leq C A^{-k(Q)}$, and so (5.107) implies that

$$
\mu\left(100 B\left(Q^{\prime}\right)\right) \leq C A^{-10\left(k\left(Q^{\prime}\right)-k(Q)\right)} A^{-k(Q)} .
$$

This was when $k\left(Q^{\prime}\right)-1 \geq k(Q)$. In this first case where (5.104) holds, the only other possibility is that $k\left(Q^{\prime}\right)=k(Q)$, and in this case (5.108) also holds, more trivially, by (4.13). So (5.108) always holds, provided that $D\left(R, R^{\prime}\right) \neq \varnothing$ and we are in Case 1 . Now we use the first half of (5.102) to get that

$$
\mu\left(V_{l}\right) \leq C A^{-10\left(l+k\left(Q^{\prime}\right)-k(Q)\right)} A^{-k(Q)} .
$$

Then we say that $\mu\left(D_{k, l}\right) \leq \min \left\{\mu\left(U_{k}\right), \mu\left(V_{l}\right)\right\}$, and use (5.98), (5.101) and (5.109) to obtain

$$
a_{k, l} \leq C A^{k+l} A^{k\left(Q^{\prime}\right)} \min \left\{A^{-10 k}, A^{-10\left(l+k\left(Q^{\prime}\right)-k(Q)\right)}\right\} .
$$

Next $\min \left\{u^{10}, v^{10}\right\} \leq u^{2} v^{8}$, and so

$$
\begin{aligned}
a_{k, l} & \leq C A^{k+l} A^{k\left(Q^{\prime}\right)} A^{-2 k} A^{-8\left(l+k\left(Q^{\prime}\right)-k(Q)\right)} \\
& =C A^{-k-7 l} A^{-7 k\left(Q^{\prime}\right)+8 k(Q)} \\
& \leq C A^{-k-l} A^{k(Q)} .
\end{aligned}
$$

(The other convergence factors will not help, and so we drop them.) Now use (5.92) and (5.97), and sum over $k$ and $l$ to get that

$$
\begin{aligned}
\left\langle e_{Q, R}, e_{Q^{\prime}, R^{\prime}}\right\rangle & \leq \mu(R) \mu\left(R^{\prime}\right) a\left(R, R^{\prime}\right) \\
& =\sum_{k} \sum_{l} \mu(R) \mu\left(R^{\prime}\right) a_{k, l} \\
& \leq C \mu(R) \mu\left(R^{\prime}\right) A^{k(Q)} .
\end{aligned}
$$


Now denote by $Z_{1}$ the set of all quadruples $\left(Q, R, Q^{\prime}, R^{\prime}\right)$ where $Q \in \mathcal{R}$, $R \in \mathcal{S}(Q), Q^{\prime} \in \mathcal{R}, R^{\prime} \in \mathcal{S}\left(Q^{\prime}\right), D\left(R, R^{\prime}\right) \neq \varnothing$ and (5.104) holds (i.e., we are in Case 1). Also set

$$
\sigma_{1}=\sum_{\left(Q, R, Q^{\prime}, R^{\prime}\right) \in Z_{1}} \theta(Q) \theta\left(Q^{\prime}\right)\left\langle e_{Q, R}, e_{Q^{\prime}, R^{\prime}}\right\rangle .
$$

Then

$$
\begin{aligned}
\sigma_{1} & \leq C \sum_{Z_{1}} \theta(Q) \theta\left(Q^{\prime}\right) \mu(R) \mu\left(R^{\prime}\right) A^{k(Q)} \\
& \leq C \sum_{Q \in \mathcal{R}} \sum_{Q^{\prime} \in \mathcal{R}(Q)} \theta(Q) \theta\left(Q^{\prime}\right) \mu(Q) \mu\left(Q^{\prime}\right) A^{k(Q)}
\end{aligned}
$$

where

$$
\mathcal{R}(Q)=\left\{Q^{\prime} \in \mathcal{R}: k\left(Q^{\prime}\right) \geq k(Q) \text { and } 2 Q \cap 2 Q^{\prime} \neq \varnothing\right\}
$$

(The last condition is needed if we want any of the sets $D\left(R, R^{\prime}\right)$ to be nonempty.) Notice that all the cubes $Q^{\prime}, Q^{\prime} \in \mathcal{R}(Q)$, are contained in a fixed ball centered on $Q$ and with radius $C A^{-k(Q)}$. Then (4.51) and (4.76) imply that

$$
\sum_{Q^{\prime} \in \mathcal{R}(Q)} \theta\left(Q^{\prime}\right) \mu\left(Q^{\prime}\right) \leq C A^{-k(Q)}
$$

and then

$$
\sigma_{1} \leq C \sum_{Q \in \mathcal{R}} \theta(Q) \mu(Q) \leq C \mu(E)
$$

by $(5.59)$.

Now we come to our second case when $k\left(Q^{\prime}\right) \geq k(Q)$, but (5.104) fails, i.e., when

$$
k\left(Q^{\prime}\right) \geq k(Q) \quad \text { and } \quad \delta>A^{-k\left(Q^{\prime}\right)+1} .
$$

In this case there is an additional constraint that we want to use: if $D_{k, l}$ is not empty, then $U_{k}$ meets $V_{l}$ and

$$
\delta=\operatorname{dist}\left(R, R^{\prime}\right) \leq A^{1-k(Q)-k}+A^{1-k\left(Q^{\prime}\right)-l} .
$$


(Compare with (5.94) and (5.95).) Since in this case $\delta>A^{-k\left(Q^{\prime}\right)+1} \gg$ $A^{-k\left(Q^{\prime}\right)-l}$ (because $l \geq 0$ ), we even have that

$$
\delta \leq 2 A^{1-k(Q)-k} .
$$

Here $\operatorname{diam} Q^{\prime}$ is much smaller than $\delta$ (by (5.118)), and hence not much larger than the "scale" $A^{-k(Q)-k}$ of $U_{k}$ (by (5.120) and the definition (5.94)). Thus it will be reasonable to derive our estimate of $\mu\left(D_{k, l}\right)$ only from the estimate (5.102) on $\mu\left(V_{l}\right)$. Let us do this. From (5.98) and (5.102) we deduce that

$$
a_{k, l} \leq C A^{k-9 l} A^{k(Q)} .
$$

Sum this first over $l$, with $k$ fixed. We get less than $C A^{k+k(Q)}$. Then sum again over all $k \geq 0$ such that (5.120) holds. This gives at most $C \delta^{-1}$. Thus (5.92) and (5.97) yield

$$
\begin{aligned}
\left\langle e_{Q, R}, e_{Q^{\prime}, R^{\prime}}\right\rangle & \leq \mu(R) \mu\left(R^{\prime}\right) a\left(R, R^{\prime}\right) \\
& =\mu(R) \mu\left(R^{\prime}\right) \sum_{k} \sum_{l} a_{k l} \\
& \leq C \mu(R) \mu\left(R^{\prime}\right) \delta^{-1} .
\end{aligned}
$$

Let $Z_{2}$ denote the set of quadruples $\left(Q, R, Q^{\prime}, R^{\prime}\right)$ with $Q \in \mathcal{R}, R \in$ $\mathcal{S}(Q), Q^{\prime} \in \mathcal{R}, R^{\prime} \in \mathcal{S}\left(Q^{\prime}\right)$, and for which (5.118) holds and $D\left(R, R^{\prime}\right) \neq$ $\varnothing$. Also set

$$
\sigma_{2}=\sum_{\left(Q, R, Q^{\prime}, R^{\prime}\right) \in Z_{2}} \theta(Q) \theta\left(Q^{\prime}\right)\left\langle e_{Q, R}, e_{Q^{\prime}, R^{\prime}}\right\rangle
$$

Then

$$
\sigma_{2} \leq \sum_{Z_{2}} \theta(Q) \theta\left(Q^{\prime}\right) \mu(R) \mu\left(R^{\prime}\right) \operatorname{dist}\left(R, R^{\prime}\right)^{-1} .
$$

Next we fix $Q \in \mathcal{R}$ and $R \in \mathcal{S}(Q)$, and we wish to sum over $Q^{\prime}$ and $R^{\prime}$. We want to compare the sum with an integral.

Lemma 5.125. If (5.118) holds and $D\left(R, R^{\prime}\right) \neq \varnothing$,

the parent $\widehat{Q}^{\prime}$ of $Q^{\prime}$ is contained in

$$
\left\{x \in E: \operatorname{dist}(x, Q) \leq C A^{-k(Q)}\right\} \text {, }
$$

$$
\operatorname{dist}\left(R, R^{\prime}\right) \geq \frac{1}{2} A^{-k(R)},
$$


and

$$
\operatorname{dist}(x, R) \leq C \operatorname{dist}\left(R, R^{\prime}\right), \quad \text { for all } x \in \widehat{Q}^{\prime}
$$

Recall from (5.91) that $D\left(R, R^{\prime}\right) \subset 2 Q \cap 2 Q^{\prime}$ and that $k\left(Q^{\prime}\right) \geq$ $k(Q)$ by (5.118). Our first assertion follows from this.

Next $D\left(R, R^{\prime}\right) \subset 2 Q^{\prime} \backslash 2 R$, and so $2 Q^{\prime}$ meets $E \backslash 2 R$ and in particular there are points in $2 Q^{\prime}$ at distance greater or equal than $A^{-k(R)}$ from $R$. On the other hand all points of $2 Q^{\prime}$ lie at distance at most $\operatorname{diam} 2 Q^{\prime}+\operatorname{dist}\left(R^{\prime}, R\right)$ from $R$, and this number is at most 2 dist $\left(R, R^{\prime}\right)$ by (5.118). This proves $(5.127)$.

Finally, if $x \in \widehat{Q}^{\prime}$, then $\operatorname{dist}(x, R) \leq \operatorname{dist}\left(x, R^{\prime}\right)+\operatorname{dist}\left(R^{\prime}, R\right) \leq$ $C A^{-k\left(Q^{\prime}\right)+1}+\operatorname{dist}\left(R^{\prime}, R\right) \leq C \operatorname{dist}\left(R, R^{\prime}\right)$ by (5.118). The lemma follows.

Lemma 5.129. For each choice of $Q$ and $R$, set

$$
\sigma(Q, R)=\sum_{Q^{\prime}} \sum_{R^{\prime}} \theta\left(Q^{\prime}\right) \mu\left(R^{\prime}\right) \operatorname{dist}\left(R, R^{\prime}\right)^{-1}
$$

where we sum over pairs $\left(Q^{\prime}, R^{\prime}\right)$ such that $\left(Q, R, Q^{\prime}, R^{\prime}\right) \in Z_{2}$. Then

$$
\sigma(Q, R) \leq C \int_{\widetilde{Q}} \varphi_{R} d \mu
$$

where $\widetilde{Q}=\left\{x \in E: \operatorname{dist}(x, Q) \leq C A^{-k(Q)}\right\}$ is the same set as in (5.126), and

$$
\varphi_{R}(x)=\left(A^{-k(R)}+\operatorname{dist}(x, R)\right)^{-1} .
$$

To prove the lemma, first observe that if $\left(Q, R, Q^{\prime}, R^{\prime}\right) \in Z_{2}$, the hypotheses of Lemma 5.125 are satisfied. Then $\operatorname{dist}\left(R, R^{\prime}\right)^{-1} \leq$ $C m_{R}\left(\widehat{Q}^{\prime}\right)$, where $m_{R}\left(\widehat{Q}^{\prime}\right)=\inf \left\{\varphi_{R}(x): x \in \widehat{Q}^{\prime}\right\}$. This follows from (5.127) and (5.128). Next

$$
\sum_{R^{\prime} \in \mathcal{S}\left(Q^{\prime}\right)} \mu\left(R^{\prime}\right) \operatorname{dist}\left(R, R^{\prime}\right)^{-1} \leq C \mu\left(Q^{\prime}\right) m_{R}\left(\widehat{Q}^{\prime}\right)
$$


Now all cubes $Q^{\prime}$ that show up in (5.130) lie in $\mathcal{A}_{n} \cup \mathcal{A}_{n}^{*}$ for some cube $Q_{n}$ such that $Q_{n} \subset \widetilde{Q}$. (This comes from (5.126).) Thus

$$
\begin{aligned}
\sigma(Q, R) & \leq C \sum_{n: Q_{n} \subset \widetilde{Q}} \sum_{Q^{\prime} \in \mathcal{A}_{n} \cup \mathcal{A}_{n}^{*}} \theta\left(Q^{\prime}\right) \mu\left(Q^{\prime}\right) m_{R}\left(\widehat{Q}^{\prime}\right) \\
& \leq C \sum_{n: Q_{n} \subset \widetilde{Q}} \mu\left(H_{n}\right) m_{R}\left(Q_{n}\right) \\
& \leq C \sum_{n: Q_{n} \subset \widetilde{Q}} \int_{H_{n}} \varphi_{R} d \mu \\
& \leq C \int_{\widetilde{Q}} \varphi_{R}(x) d \mu(x)
\end{aligned}
$$

with a definition of $m_{R}\left(Q_{n}\right)$ similar to the definition of $m_{R}\left(\widehat{Q}^{\prime}\right)$ above, and by (4.75) and the disjointness property (4.69) for the $H_{n}$ 's. This proves the lemma.

Our next task is to estimate the integral in (5.131). As usual, we cut it in slices and use the fact that there is no good cube between $R$ and $Q$. Let us start with the contribution of the "annuli" $U_{k}, 0 \leq k \leq$ $k(R)-k(Q)+1$, from (5.94). We can use (5.101) to get that

$$
\int_{U_{k}} \varphi_{R} d \mu \leq A^{k(Q)+k} \mu\left(U_{k}\right) \leq C A^{-9 k}
$$

The contribution of the region near $R$ can be estimated in the same way. If $D_{0}=\left\{x \in E: \operatorname{dist}(x, R) \leq A^{-k(R)-1}\right\}$, then $\varphi_{R}(x) \leq A^{k(R)}$ on $D_{0}$ (and even everywhere), and $D_{0} \subset 100 B(R)$. Then

$$
\begin{aligned}
\mu\left(D_{0}\right) & \leq \mu(100 B(R)) \\
& \leq C A^{-10(k(R)-k(Q))} \mu(100 B(Q)) \\
& \leq C A^{-10 k(R)+9 k(Q)}
\end{aligned}
$$

by Lemma 5.31 and the density estimate (4.13), applied to $Q$ or its parent. Thus

$$
\int_{D_{0}} \varphi_{R} d \mu \leq A^{k(R)} \mu\left(D_{0}\right) \leq C A^{-9(k(R)-k(Q))} .
$$


For the exterior "annulus" $D_{\infty}=\left\{x \in \widetilde{Q}: \operatorname{dist}(x, R)>A^{-k(Q)}\right\}$, we use (4.13) again (applied to an ancester of $Q$, possibly its grandparent) to get that $\mu(\widetilde{Q}) \leq C A^{-k(Q)}$, and then

$$
\int_{D_{\infty}} \varphi_{R} d \mu \leq C
$$

From (5.135), (5.136), (5.137) and the fact that

$$
\widetilde{Q}=D_{\infty} \cup D_{0} \cup\left(\bigcup_{k=0}^{k(R)-k(Q)} U_{k}\right)
$$

we deduce that

$$
\int_{\widetilde{Q}} \varphi_{R} d \mu \leq C
$$

and then Lemma 5.129 tells us that $\sigma(Q, R) \leq C$. If we compare (5.124) with the definition (5.130), we obtain that

$$
\sigma_{2} \leq C \sum_{Q \in \mathcal{R}} \sum_{R \in \mathcal{S}(Q)} \theta(Q) \mu(R) \leq C \sum_{Q \in \mathcal{R}} \theta(Q) \mu(Q) \leq C \mu(E)
$$

by (5.59). Note that $\sigma_{1}$ and $\sigma_{2}$ control all the terms where $k\left(Q^{\prime}\right) \geq$ $k(Q)$, and so

$$
\begin{aligned}
\left\|W_{4}\right\|_{L^{2}(d \mu)}^{2} & =\left\|\sum_{Q \in \mathcal{R}} \sum_{R \in \mathcal{S}(Q)} \theta(Q) e_{Q, R}\right\|_{2}^{2} \\
& \leq 2 \sigma_{1}+2 \sigma_{2} \\
& \leq C \mu(E)
\end{aligned}
$$

by the definition (5.87) of $W_{4}$, the definitions (5.113) and (5.123) of $\sigma_{1}$ and $\sigma_{2}$, and the estimates (5.117) and (5.139). This completes our study of the function $W_{4}$. 


\subsection{The very local part $W_{5}$.}

We are left with

$$
W_{5}=\sum_{Q \in \mathcal{R}} \sum_{R \in \mathcal{S}(Q)} \theta(Q) e_{Q, R}^{0}
$$

with $e_{Q, R}^{0}$ as in (5.85). We start with an estimate of $e_{Q, R}^{0}$ for $Q$ and $R$ fixed. Of course we may assume that $x \in 2 R \backslash Q$, since otherwise $e_{Q, R}^{0}(x)=0$. Set

$$
d_{R}(x)=A^{k(R)} \operatorname{dist}(x, R) .
$$

Lemma 5.142. We have that

$$
e_{Q, R}^{0}(x) \leq C d_{R}(x)^{-1 / 10} A^{-9(k(R)-k(Q))} .
$$

Before we prove the lemma, observe that we could have proved an estimate with only a logarithmic singularity in $d_{R}(x)$, but we prefer (5.143) because of the convergence factor $A^{-9(k(R)-k(Q))}$.

To prove the lemma, recall first from (5.26) that $\mu(\bar{Q} \backslash Q)=0$. Let $x \in 2 R \backslash \bar{Q}$ be given and denote by $l(x)$ the largest integer $l$ such that $x \in N_{l}(R)$, where $N_{l}(R)$ is as in (5.25) (or Chapter 3). Note that the only case when $x \notin N_{0}(R)$ is when dist $(x, R)=A^{-k(Q)}$, because $x \in 2 R$. In this case we still set $l(x)=0$. In all events

$$
A^{-k(R)-l(x)-1} \leq \operatorname{dist}(x, R) \leq A^{-k(R)-l(x)} .
$$

We split the domain of integration $R$ into regions

$$
D_{k}=\left\{y \in R: A^{-k} \leq|x-y|<A^{-k+1}\right\} \text {. }
$$

Let us check first that if $D_{k}$ is not empty, then

$$
k(R) \leq k \leq k(R)+l(x)+1 .
$$

If $y \in R$, then $|x-y|<A^{-k(R)+1}$ because $x \in 2 R$, and so $A^{-k}<$ $A^{-k(R)+1}$ if $D_{k}$ is not empty; this proves the first inequality. Similarly, the first inequality in (5.144) forces $A^{-k(R)-l(x)-1}<A^{-k+1}$ if $D_{k}$ is not empty; this completes the proof of (5.146). 
Next $d(y, E \backslash R) \leq|x-y|<A^{-k+1}$ for $y \in D_{k}$, and so $D_{k} \subset$ $N_{k-k(R)-1}(R)$ if $k>k(R)$. (If $k=k(R)$, we shall simply remember that $D_{k} \subset R$.) Then

$$
\int_{D_{k}} \frac{d \mu(y)}{|x-y|} \leq A^{k} \mu\left(D_{k}\right) \leq C A^{k} C_{0}^{-93(k-k(R))} \mu(90 B(R))
$$

by (5.26). Recall from (4.1) that $A=C C_{0}^{100}$ for some absolute constant $C$, and so $A C_{0}^{-93}=C C_{0}^{7} \leq C^{1 / 10} C_{0}^{10}=A^{1 / 10}$ if $C_{0}$ is large enough, which we are happy to assume. Because of this,

$$
\begin{aligned}
\int_{D_{k}} \frac{d \mu(y)}{|x-y|} & \leq A^{(k-k(R)) / 10} A^{k(R)} \mu(90 B(R)) \\
& \leq C A^{(k-k(R)) / 10} A^{-9(k(R)-k(Q))} A^{k(Q)} \mu(100 B(Q)) \\
& \leq C A^{(k-k(R)) / 10} A^{-9(k(R)-k(Q))}
\end{aligned}
$$

by Lemma 5.31, the fact that $R$ is a maximal good subcube of $Q$, and the usual density estimate (4.13) applied to $Q$ or its parent.

When we sum (5.148) over $k$, we get a geometric series whose leading term is when $k$ is largest, i.e., $k=k(R)+l(x)+1$. Also note that $A^{-l(x)} \sim d_{R}(x)$ by (5.144). Thus

$$
\begin{aligned}
e_{Q, R}^{0}(x) & =\int_{R} \frac{d \mu(y)}{|x-y|}=\sum_{k=k(R)}^{k(R)+l(x)+1} \int_{D_{k}} \frac{d \mu(y)}{|x-y|} \\
& \leq C A^{l(x) / 10} A^{-9(k(R)-k(Q))} \\
& \leq C d_{R}(x)^{-1 / 10} A^{-9(k(R)-k(Q))} .
\end{aligned}
$$

This proves Lemma 5.142 .

Now we fix the cubes $Q, Q^{\prime}, R, R^{\prime}$, and we estimate

$$
I\left(R, R^{\prime}\right)=\left\langle e_{Q, R}^{0}, e_{Q^{\prime}, R^{\prime}}^{0}\right\rangle=\int_{D\left(R, R^{\prime}\right)} e_{Q, R}^{0} e_{Q^{\prime}, R^{\prime}}^{0} d \mu
$$

where

$$
D\left(R, R^{\prime}\right)=\left(2 R \cap 2 R^{\prime}\right) \backslash\left(Q \cup Q^{\prime}\right) .
$$


Obviously by Lemma 5.142

$$
I\left(R, R^{\prime}\right) \leq C A^{-9\left(k(R)-k(Q)+k\left(R^{\prime}\right)-k\left(Q^{\prime}\right)\right)} J\left(R, R^{\prime}\right),
$$

where

$$
J\left(R, R^{\prime}\right)=\int_{D\left(R, R^{\prime}\right)}\left(d_{R}(x) d_{R^{\prime}}(x)\right)^{-1 / 10} d \mu(x)
$$

and $d_{R^{\prime}}$ is defined like $d_{R}\left(\right.$ i.e., $d_{R^{\prime}}(x)=A^{k\left(R^{\prime}\right)} \operatorname{dist}\left(x, R^{\prime}\right)$.)

Lemma 5.154. Suppose for definiteness that $k\left(R^{\prime}\right) \geq k(R)$. Then

$$
J\left(R, R^{\prime}\right) \leq C\left(\frac{A^{-k(R)}}{A^{-k\left(R^{\prime}\right)}+\operatorname{dist}\left(R, R^{\prime}\right)}\right)^{1 / 10} \mu\left(R^{\prime}\right) .
$$

We start the proof of Lemma 5.154 in the case when

$$
\operatorname{dist}\left(R, R^{\prime}\right) \geq \operatorname{diam} R^{\prime}+A^{-k\left(R^{\prime}\right)} .
$$

In this case the function $d_{R}(x)$ is essentially constant on $D\left(R, R^{\prime}\right)$ (because $\left.D\left(R, R^{\prime}\right) \subset 2 R^{\prime}\right)$ and

$$
\begin{aligned}
& J\left(R, R^{\prime}\right) \\
& \quad \leq C\left(A^{k(R)} \operatorname{dist}\left(R, R^{\prime}\right)\right)^{-1 / 10} \int_{D\left(R, R^{\prime}\right)} d_{R^{\prime}}(x)^{-1 / 10} d \mu(x) .
\end{aligned}
$$

The last integral is easy to estimate. Since we are going to need a variant later, let us state a slightly more general lemma.

Lemma 5.158. For each cube $R \in \Delta$, set

$$
\delta_{R}(x)=A^{k(R)}(\operatorname{dist}(x, R)+\operatorname{dist}(x, E \backslash R)),
$$

for all $x \in E \cap 100 B(R)$. Then

$$
\int_{E \cap 100 B(R)} \delta_{R}(x)^{-1 / 5} d \mu(x) \leq C \mu(100 B(R))
$$


To prove the lemma, we use the sets $N_{l}(R)$ of (5.25) and Chapter 3 . For each $l \geq 0$,

$$
\begin{aligned}
\int_{N_{l}(R) \backslash N_{l+1}(R)} \delta_{R}(x)^{-1 / 5} d \mu(x) & \leq A^{(l+1) / 5} \mu\left(N_{l}(R)\right) \\
& \leq C A^{l / 5} C_{0}^{-93 l} \mu(90 B(R)) \\
& \leq C\left(C^{\prime} C_{0}^{20} C_{0}^{-93}\right)^{l} \mu(90 B(R))
\end{aligned}
$$

by (5.26), and because $A=C^{\prime 5} C_{0}^{100}$ for some absolute constant $C^{\prime}$, as in (4.1). We may sum over $l \geq 0$ and get that

$$
\int_{N_{0}(R)} \delta_{R}(x)^{-1 / 5} d \mu(x) \leq C \mu(90 B(R))
$$

(The set where $\delta_{R}(x)=0$ has measure zero by (5.26), so we can forget about it.)

On the rest of $E \cap 100 B(R)$, that is, on $(E \cap 100 B(R)) \backslash N_{0}(R)$, $\delta_{R}(x) \geq C^{-1}$ and the corresponding integral is at most $C \mu(100 B(R))$. The lemma follows.

Notice that $\delta_{R}=d_{R}$ on $2 R \backslash R$, and so it follows from (5.157) and Lemma 5.158 (applied to $R^{\prime}$ ) that

$$
J\left(R, R^{\prime}\right) \leq C\left(A^{k(R)} \operatorname{dist}\left(R, R^{\prime}\right)\right)^{-1 / 10} \mu\left(100 B\left(R^{\prime}\right)\right) .
$$

This is less than the right-hand side of (5.155) because of (5.156) and the fact that $\mu\left(100 B\left(R^{\prime}\right)\right) \leq C_{0} \mu\left(R^{\prime}\right)$ since $R^{\prime}$ is a good cube. This proves (5.155) when (5.156) holds.

Now assume that dist $\left(R, R^{\prime}\right)<\operatorname{diam} R^{\prime}+A^{-k\left(R^{\prime}\right)}$. Cover $2 R^{\prime} \backslash(R \cup$ $R^{\prime}$ ) by the cubes $S$ of generation $k\left(R^{\prime}\right)+1$ that meet $2 R^{\prime} \backslash\left(R \cup R^{\prime}\right)$. Note that $100 B(S) \subset 100 B\left(R^{\prime}\right)$ for each such cube $S$, and also that we need less than $C$ cubes $S$. If $x \in S, d_{R}(x) d_{R^{\prime}}(x) \geq A^{k(R)} A^{k\left(R^{\prime}\right)} \operatorname{dist}(x, E \backslash$ $S)^{2}$, because $S$ does not meet $R$ or $R^{\prime}$. (Recall that $k(S)>k\left(R^{\prime}\right) \geq$ $k(R)$.) Thus

$$
\begin{aligned}
& \int_{D\left(R, R^{\prime}\right)}\left(d_{R} d_{R^{\prime}}\right)^{-1 / 10} d \mu \\
& \leq A^{-\left(k(R)+k\left(R^{\prime}\right)\right) / 10} \sum_{S} \int_{S} \operatorname{dist}(x, E \backslash S)^{-1 / 5} d \mu(x)
\end{aligned}
$$




$$
\begin{aligned}
& \leq C A^{-\left(k(R)+k\left(R^{\prime}\right)\right) / 10} \sum_{S} A^{k\left(R^{\prime}\right) / 5} \mu(100 B(S)) \\
& \leq C A^{-\left(k(R)-k\left(R^{\prime}\right)\right) / 10} \mu\left(100 B\left(R^{\prime}\right)\right) \\
& \leq C A^{-\left(k(R)-k\left(R^{\prime}\right)\right) / 10} \mu\left(R^{\prime}\right)
\end{aligned}
$$

by Lemma 5.158 (applied to each $S$ ), and the fact that $R^{\prime}$ is a good cube. This time $A^{-k\left(R^{\prime}\right)}+\operatorname{dist}\left(R, R^{\prime}\right) \sim A^{-k\left(R^{\prime}\right)}$ because dist $\left(R, R^{\prime}\right)<$ $\operatorname{diam} R^{\prime}+A^{-k\left(R^{\prime}\right)}$, and (5.155) follows from (5.163). This completes our proof of Lemma 5.154 .

Now let us fix $Q, Q^{\prime}$ and $R$, and try to sum $I\left(R, R^{\prime}\right)$ over all cubes $R^{\prime} \in S\left(Q^{\prime}\right)$. We want to do a comparison with an integral. If $R^{\prime} \in \mathcal{S}\left(Q^{\prime}\right)$ and $x$ is any point of $\widehat{Q}^{\prime}$ (the parent of $Q^{\prime}$ ), and if $D\left(R, R^{\prime}\right)$ is not empty,

$$
\begin{aligned}
\operatorname{dist} & (x, R)+\operatorname{dist}(x, E \backslash R) \\
& \leq \operatorname{dist}\left(x, R^{\prime}\right)+\operatorname{diam} R^{\prime}+\operatorname{dist}\left(R^{\prime}, R\right)+\operatorname{dist}(x, E \backslash R) \\
& \leq C A^{-k\left(Q^{\prime}\right)}+\operatorname{dist}\left(R^{\prime}, R\right)+\operatorname{dist}(x, E \backslash R) \\
& \leq C A^{-k\left(Q^{\prime}\right)}+\operatorname{dist}\left(R, R^{\prime}\right)+\operatorname{dist}\left(x, D\left(R, R^{\prime}\right)\right) \\
& \leq C A^{-k\left(Q^{\prime}\right)}+\operatorname{dist}\left(R, R^{\prime}\right)+C A^{-k\left(Q^{\prime}\right)} \\
& \leq C A^{k\left(R^{\prime}\right)-k\left(Q^{\prime}\right)}\left(A^{-k\left(R^{\prime}\right)}+\operatorname{dist}\left(R, R^{\prime}\right)\right)
\end{aligned}
$$

because $R^{\prime} \subset Q^{\prime}$, and $D\left(R, R^{\prime}\right)=\left(2 R \cap 2 R^{\prime}\right) \backslash\left(Q \cup Q^{\prime}\right)$ is contained in $E \backslash R$ and meets $2 Q^{\prime}$. With the notation of Lemma 5.158,

$$
\delta_{R}(x) \leq C A^{k\left(R^{\prime}\right)-k\left(Q^{\prime}\right)}\left(\frac{A^{-k\left(R^{\prime}\right)}+\operatorname{dist}\left(R, R^{\prime}\right)}{A^{-k(R)}}\right)
$$

for all $x \in \widehat{Q}^{\prime}$. Now suppose that

$$
\widehat{Q}^{\prime} \subset 100 B(R)
$$

and let $\mathcal{S}_{R}\left(Q^{\prime}\right)$ denote the set of cubes $R^{\prime} \in \mathcal{S}\left(Q^{\prime}\right)$ such that $k\left(R^{\prime}\right) \geq$ $k(R)$. (We need not prove that (5.166) implies that $k\left(Q^{\prime}\right) \geq k(R)$, so 
let us not bother.) Then

$\sum_{R^{\prime} \in \mathcal{S}_{R}\left(Q^{\prime}\right)} I\left(R, R^{\prime}\right)$

$$
\begin{aligned}
& \leq C \sum_{R^{\prime}} A^{-9\left(k\left(R^{\prime}\right)-k\left(Q^{\prime}\right)\right)}\left(\frac{A^{-k(R)}}{A^{-k\left(R^{\prime}\right)}+\operatorname{dist}\left(R, R^{\prime}\right)}\right)^{1 / 10} \mu\left(R^{\prime}\right) \\
& \leq C \sum_{R^{\prime}} \inf \left\{\delta_{R}(x)^{-1 / 10}: x \in \widehat{Q}^{\prime}\right\} \mu\left(R^{\prime}\right) \\
& \leq C \inf \left\{\delta_{R}(x)^{-1 / 10}: x \in \widehat{Q}^{\prime}\right\} \mu\left(Q^{\prime}\right)
\end{aligned}
$$

by $(5.152),(5.155)$ and (5.165). We have dropped most of the convergence factor in (5.152) because it will no longer be useful here. Now we want to sum over $Q^{\prime}$ as well. Set

$$
I_{1}(R)=\sum_{Q^{\prime}} \sum_{R^{\prime}} \theta\left(Q^{\prime}\right) I\left(R, R^{\prime}\right),
$$

where we sum on pairs $Q^{\prime}, R^{\prime}$ such that (5.166) holds and $k\left(R^{\prime}\right) \geq k(R)$. For each cube $Q_{n}$ such that $Q_{n} \subset 100 B(R)$,

$$
\begin{aligned}
\sum_{Q^{\prime} \in \mathcal{A}_{n} \cup \mathcal{A}_{n}^{*}} \theta\left(Q^{\prime}\right) \mu\left(Q^{\prime}\right) \inf \left\{\delta_{R}(x)^{-1 / 10}: x \in Q_{n}\right\} \\
\leq C \mu\left(H_{n}\right) \inf \left\{\delta_{R}(x)^{-1 / 10}: x \in H_{n}\right\} \\
\leq C \int_{H_{n}} \delta_{R}(x)^{-1 / 10} d \mu(x)
\end{aligned}
$$

by definitions, and (4.33) when $Q_{n} \in$ PLI. Then

$$
\begin{aligned}
I_{1}(R) & \leq C \sum_{n: Q_{n} \subset 100 B(R)} \int_{H_{n}} \delta_{R}^{-1 / 10} d \mu \\
& \leq C \int_{100 B(R)} \delta_{R}^{-1 / 10} d \mu \\
& \leq C \mu(100 B(R)) \\
& \leq C \mu(R)
\end{aligned}
$$

because all the cubes $Q^{\prime}$ for which (5.166) holds lie in $\mathcal{A}_{n} \cup \mathcal{A}_{n}^{*}$ for some $Q_{n}$ such that $Q_{n} \subset \widehat{Q}^{\prime} \subset 100 B(R)$, by (5.167), (5.169), Lemma 5.158, and the fact that $R$ is a good cube. 
Let $Z_{1}$ be the set of all quadruples $\left(Q, R, Q^{\prime}, R^{\prime}\right)$ for which $Q$, $Q^{\prime} \in \mathcal{R}, R \in \mathcal{S}(Q), R^{\prime} \in \mathcal{S}\left(Q^{\prime}\right)$, (5.166) holds, and $k\left(R^{\prime}\right) \geq k(R)$. Then

$$
\begin{aligned}
\sum_{Z_{1}} \theta(Q) \theta\left(Q^{\prime}\right)\left\langle e_{Q, R}^{0}, e_{Q^{\prime}, R^{\prime}}^{0}\right\rangle & =\sum_{Z_{1}} \theta(Q) \theta\left(Q^{\prime}\right) I\left(R, R^{\prime}\right) \\
& \leq C \sum_{Q \in \mathcal{R}} \sum_{R \in \mathcal{S}(Q)} \theta(Q) \mu(R) \\
& \leq C \sum_{Q \in \mathcal{R}} \theta(Q) \mu(Q) \\
& \leq C \mu(E)
\end{aligned}
$$

by (5.150), (5.168), (5.170), and (5.59).

Now let $Z_{2}$ denote the set of quadruples $\left(Q, R, Q^{\prime}, R^{\prime}\right)$ with $Q$, $Q^{\prime} \in \mathcal{R}, R \in \mathcal{S}(Q), R^{\prime} \in \mathcal{S}\left(Q^{\prime}\right)$, and $k\left(R^{\prime}\right) \geq k(R)$ as before, but (5.166) does not hold.

If (5.166) does not hold and $D\left(R, R^{\prime}\right) \neq \varnothing$, then $k\left(Q^{\prime}\right) \leq k(R)+1$ because $\widehat{Q}^{\prime}$ is not contained in $100 B(R)$ while $2 Q^{\prime}$ meets $2 R$. Then

$$
\frac{A^{-k(R)}}{A^{-k\left(R^{\prime}\right)}+\operatorname{dist}\left(R, R^{\prime}\right)} \leq A^{k\left(R^{\prime}\right)-k(R)} \leq A^{k\left(R^{\prime}\right)-k\left(Q^{\prime}\right)+1},
$$

and we deduce from (5.155) that $J\left(R, R^{\prime}\right) \leq C A^{k\left(R^{\prime}\right)-k\left(Q^{\prime}\right)} \mu\left(R^{\prime}\right)$ and then from (5.152) that

$$
I\left(R, R^{\prime}\right) \leq C A^{-k\left(R^{\prime}\right)+k\left(Q^{\prime}\right)} \mu\left(R^{\prime}\right) .
$$

(Here again we may drop most of the convergence factor.) Because $D\left(R, R^{\prime}\right) \subset 2 R \cap 2 R^{\prime}$ and $k\left(R^{\prime}\right) \geq k(R)$, we only need to consider cubes $R^{\prime}$ that are contained in $100 B(R)$.

For a given cube $R^{\prime} \subset 100 B(R)$, the sum over all cubes $Q^{\prime}$ of generations at most $k(R)+1$ of the right-hand side of (5.173) is less than $C A^{-k\left(R^{\prime}\right)+k(R)} \mu\left(R^{\prime}\right)$, because there is at most one cube $Q^{\prime}$ per generation. If we sum this bound over all cubes $R^{\prime}$ of a given generation $k^{\prime} \geq k(R)$, we get less than $C A^{-k^{\prime}+k(R)} \mu(100 B(R))$, and if we sum again over all possible generations $k^{\prime} \geq k(R)$, we get less than $C \mu(100 B(R)) \leq C \mu(R)$ because $R$ is a good cube. Thus for each $Q \in \mathcal{R}$ and each $R \in \mathcal{S}(Q)$,

$$
\sum_{Q^{\prime}} \sum_{R^{\prime}} I\left(R, R^{\prime}\right) \leq C \mu(R)
$$


where we sum over all pairs $Q^{\prime}, R^{\prime}$ such that $\left(Q, R, Q^{\prime}, R^{\prime}\right) \in Z_{2}$. Hence

$$
\begin{aligned}
\sum_{Z_{2}} \theta(Q) \theta\left(Q^{\prime}\right)\left\langle e_{Q, R}^{0}, e_{Q^{\prime}, R^{\prime}}^{0}\right\rangle & =\sum_{Z_{2}} \theta(Q) \theta\left(Q^{\prime}\right) I\left(R, R^{\prime}\right) \\
& \leq C \sum_{Q \in \mathcal{R}} \sum_{R \in \mathcal{S}(Q)} \theta(Q) \mu(R) \\
& \leq C \sum_{Q \in \mathcal{R}} \theta(Q) \mu(Q) \\
& \leq C \mu(E)
\end{aligned}
$$

by (5.150), (5.174), and (5.59).

When we collect the sum over $Z_{1} \cup Z_{2}$, we get all the terms for which $k\left(R^{\prime}\right) \geq k(R)$; the other ones can of course be obtained by symmetry, and so

$$
\left\|W_{5}\right\|_{L^{2}(d \mu)}^{2}=\left\|\sum_{Q \in \mathcal{R}} \sum_{R \in \mathcal{S}(Q)} \theta(Q) e_{Q, R}^{0}\right\|^{2} \leq C \mu(E)
$$

by (5.171) and (5.175).

This completes our estimate of the last piece of $W$. (See (5.82), (5.89), (5.140) and now (5.176) for the control of $W$.) Since we have seen also that $W$ controls $W_{2}$ and $\widetilde{W}_{2}$ (see (5.64) and (5.72)), we get the desired bounds on these functions as well. Thus the proof of Theorem 2.4 is complete.

REMARK 5.177. Suppose we give ourselves a set $J \subset \mathbb{N}^{*}$ and define functions $W_{1}^{J}, \widetilde{W}_{1}^{J}, W_{2}^{J}$ and $\widetilde{W}_{2}^{J}$ as in (5.1)-(5.4), but where we sum only on those cubes $Q \in \mathcal{R}$ that lie in $\mathcal{A}_{n} \cup \mathcal{A}_{n}^{*}$ for some $n \in J$. A close look at the arguments in this chapter shows that we also get that

$$
\begin{aligned}
& \left\|W_{1}^{J}\right\|_{L^{2}(d \nu)}^{2}+\left\|\widetilde{W}_{1}^{J}\right\|_{L^{2}(d \nu)}^{2}+\left\|W_{2}^{J}\right\|_{L^{2}(d \nu)}^{2}+\left\|\widetilde{W}_{2}^{J}\right\|_{L^{2}(d \nu)}^{2} \\
& \quad \leq C \sum_{n \in J} \mu\left(H_{n}\right) \\
& \quad \leq C \mu\left(\bigcup_{n \in J} Q_{n}\right)
\end{aligned}
$$

where the last inequality comes from (4.69). The proof is the same; we simply have to replace the usual estimate that $\sum_{\mathcal{R}} \theta(Q) \mu(Q) \leq$ 
$C \mu(E)$ at the end of our various estimates (i.e., in (5.60), (5.81), (5.117), (5.171) and (5.175)) with the corresponding fact that

$$
\sum_{n}\left(\sum_{\mathcal{A}_{n} \cup \mathcal{A}_{n}^{*}} \theta(Q) \mu(Q)\right) \leq C \sum_{n} \mu\left(H_{n}\right) .
$$

REMARK 5.179. In our $L^{2}$-estimates we have used the measure $\nu$, but we could also have used the following slightly larger measure

$$
\begin{aligned}
\nu^{+} & =\mathbf{1}_{E_{\infty}} d \mu+\sum_{n \geq 1}\left(\rho_{n}^{*}\right)^{-1} d \nu_{n} \\
& =\mathbf{1}_{E_{\infty}} d \mu+\sum_{n \geq 1} \frac{\mu\left(H_{n}\right)}{H^{1}\left(\mathcal{C}_{n}\right)} d H^{1} \mid \mathcal{C}_{n},
\end{aligned}
$$

where $\rho_{n}^{*}$ denotes the constant value of $\rho_{n-1}$ on $Q_{n}$, as in the definitions (4.17) and (4.26) of $\nu_{n}$. (Compare (5.180) with the definition (4.41) of $\nu$.$) Note that$

$$
\left(\rho_{n}^{*}\right)^{-1}\left\|\nu_{n}\right\|=\mu\left(H_{n}\right)
$$

by definitions (or by (4.21) and (4.37)).

We claim that our proof also allows the same control on the norms of $W_{1}, \widetilde{W}_{1}, W_{2}$ and $\widetilde{W}_{2}$ in $L^{2}\left(d \nu^{+}\right)$, and similarly that

$$
\begin{aligned}
& \left\|W_{1}^{J}\right\|_{L^{2}\left(d \nu^{+}\right)}^{2}+\left\|\widetilde{W}_{1}^{J}\right\|_{L^{2}\left(d \nu^{+}\right)}^{2}+\left\|W_{2}^{J}\right\|_{L^{2}\left(d \nu^{+}\right)}^{2}+\left\|\widetilde{W}_{2}^{J}\right\|_{L^{2}\left(d \nu^{+}\right)}^{2} \\
& \quad \leq C \sum_{n \in J} \mu\left(H_{n}\right) \\
& \quad \leq C \mu\left(\bigcup_{n \in J} Q_{n}\right)
\end{aligned}
$$

This time we only have to recall the way we obtained our control on $W_{1}, \widetilde{W}_{1}, W_{2}$ and $\widetilde{W}_{2}$ in terms of the $L^{2}$-norms (for $d \mu$ ) of the functions $W_{3}$ and $W$ defined in (5.9) and (5.63). For $W_{1}$, we only noticed that $h_{Q}(x)=\mathbf{1}_{Q}(x) h_{Q}^{*}(x)$ for $x \in E_{\infty}$, so that $W_{1}=W_{3}$ on $E_{\infty}$. Thus $\left\|W_{1}\right\|_{L^{2}\left(d \nu^{+}\right)}^{2} \leq\left\|W_{3}\right\|_{L^{2}(d \mu)}^{2}$ directly (i.e., without using the weight $\rho_{\infty}$ ). As for $\widetilde{W}_{1}$, let us note that the proof of Lemma 5.10 only uses the information that $\left\|\nu_{n}\right\| \leq \mu\left(H_{n}\right)$, and not any more precise informations 
that would have used the fact that $\left\|\nu_{n}\right\|=\rho_{n}^{*} \mu\left(H_{n}\right)$ and the precise size of $\rho_{n}^{*}$. Thus we may replace $\nu$ with $\nu^{+}$in Lemma 5.10 and use (5.181). Similar remarks apply to our control on $W_{2}$ and $\widetilde{W}_{2}$ using the function $W$ : we only used the fact that $W_{2}(x)=W(x)$ on $E_{\infty}$ (and not the size of $\rho_{\infty}$ on $E_{\infty}$ ) and the fact that $\left\|\nu_{n}\right\| \leq \mu\left(H_{n}\right)$ (and not the precise value of $\rho_{n}^{*}$ ). Except for these initial reductions (i.e., Lemma 5.10, (5.64) and (5.72)), the argument of Chapter 5 does not need to be modified; we were only working with the measure $\mu$. The same modifications also work with $W_{1}^{J}$ and its colleagues, and gives (5.182).

Remarks 5.177 and 5.179 are probably the first step towards a BMO-type estimate on the $T^{\varepsilon}(g d \nu)$.

\section{References.}

[C1] Christ, M., Lectures on Singular Integral Operators. Regional Conference Series in Mathematics 77, Amer. Math. Soc., 1990.

[C2] Christ, M., A $T(b)$ theorem with remarks on analytic capacity and the Cauchy integral. Colloq. Math. 60/61 (1990), 601-628.

[D1] David, G., Opérateurs intégraux singuliers sur certaines courbes du plan complexe. Ann. Sci. École Norm. Sup. 17 (1984), 157-189.

[D2] David, G., Wavelets and Singular Integrals on Curves and Surfaces. Lecture Notes in Math. 1465, Springer-Verlag, 1991.

[D3] David, G., Unrectifiable 1-sets have vanishing analytic capacity. Revista Mat. Iberoamericana 14 (1998), 369-479.

[F] Farag, H., The Riesz kernels do not give rise to higher dimensional analogues of the Menger-Melnikov curvature. Publ. Mat. 43 (1999), 251-260.

[G] Garnett, J., Analytic Capacity and Measure. Lecture Notes in Math. 297, Springer-Verlag, 1972.

[L] Léger, J.-C., Menger curvature and rectifiability. Ann. of Math. 149 (1999), 831-869.

[M] Mattila, P., Geometry of Sets and Measures in Euclidean Spaces. Cambridge Studies in Advanced Mathematics 44, Cambridge University Press, 1995.

[MMV] Mattila, P., Melnikov, M. S., Verdera, J., The Cauchy integral, analytic capacity, and uniform rectifiability. Ann. of Math. 144 (1996), 127-136.

[MP] Mattila, P., Paramonov, P. V., On geometric properties of harmonic Lip ${ }_{1}$-capacity. Pacific J. Math. 171 (1995), 469-491. 
[Me] Melnikov, M. S., Analytic capacity: discrete approach and curvature of measure. Sbornik: Mathematics 186 (1995), 827-846.

[MV] Melnikov, M. S., Verdera, J., A geometric proof of the $L^{2}$ boundedness of the Cauchy integral on Lipschitz graphs. Inter. Math. Res. Not. 7 (1995), 325-331.

[NTV] Nazarov, F., Treil, S., Volberg, A., Pulling ourselves by the hair (the proof of the Vitushkin conjecture). Preprint, Michigan State University.

[O] O'Neil, T., A local version of the projection theorem. Proc. London Math. Soc. 73 (1996), 68-104.

[S] Stein, E. M., Singular Integrals and Differentiability Properties of Functions. Princeton University Press, 1970.

[U1] Uy, N. X., An extremal problem on singular integrals. Amer. J. Math. 102 (1980), 279-290.

[U2] Uy, N. X., A removable set for Lipschitz harmonic functions. Michigan Math. J. 37 (1990), 45-51.

Recibido: 12 de abril de 1.999

Guy David

Mathématiques

Bât. 425

Université de Paris-Sud

F-91405 Orsay Cedex, FRANCE

et

Institut Universitaire de France

Guy.David@math.u-psud.fr

and

Pertti Mattila

Department of Mathematics

University of Jyväskylä

P.O. Box 35

FIN-40351 Jyväskylä, FINLAND

pmattila@math.jyu.fi 